\title{
Age-related Immune-Stromal Networks Inhibit Response to Regenerative Immunotherapies
}

Jin Han $^{1}$, Christopher Cherry ${ }^{1}$, Anna Ruta ${ }^{1}$, David R. Maestas Jr. ${ }^{1}$, Joscelyn C. Mejias ${ }^{1}$, Helen Hieu Nguyen ${ }^{1}$, Elana J. Fertig ${ }^{2,3,4}$, Franck Housseau ${ }^{5}$, Sudipto Ganguly ${ }^{5}$, Erika M. Moore ${ }^{6}$, Ada J. $\operatorname{Tam}^{5}$, Drew M. Pardoll ${ }^{5}$, and Jennifer H. Elisseeff ${ }^{1,5, *}$

${ }^{1}$ Translational Tissue Engineering Center, Wilmer Eye Institute and Department of Biomedical Engineering, Johns Hopkins University, Baltimore, MD 21287, USA; ${ }^{2}$ Department of Biomedical Engineering and Institute for Cell Engineering, Johns Hopkins University School of Medicine, Baltimore, MD, USA; ${ }^{3}$ Department of Oncology, Johns Hopkins University School of Medicine, Baltimore, MD, USA; ${ }^{4}$ Department of Applied Mathematics and Statistics, Johns Hopkins University, Baltimore, MD, USA; ${ }^{5}$ Bloomberg Kimmel Institute for Cancer Immunotherapy, Sidney Kimmel Comprehensive Cancer Center, Johns Hopkins University School of Medicine,

Baltimore, MD 21231, USA; ${ }^{6}$ Department of Materials Science and Engineering, University of Florida, Gainesville, FL USA.

*Address for correspondence: jhe@jhu.edu 
Aging is associated with immunological changes that compromise response to infections and vaccines, exacerbate inflammatory diseases and could potentially mitigate tissue repair. Indeed, regenerative medicine strategies designed to promote tissue repair are now focusing on the immune system as a therapeutic target due to its role in response to tissue damage and regulation of tissue repair. However, age-related immune changes to the response to damage and the resulting impact on repair remains unknown. Here, we characterized age-related immunological changes that inhibit tissue repair and therapeutic response to a clinical regenerative biological scaffold derived from extracellular matrix (ECM). We found that aging reduced the response of interleukin (IL)4 producing eosinophils and CD4 T cells in a volumetric muscle injury treated with ECM leading to reduced repair and increased fibrosis. Single cell RNA sequencing and cell-cell communication analysis via transcription factor (TF) activation revealed diminished interactions between immune and stromal modules in aging animals. Validation of the age-specific TFs and predicated protein interactions in the tissue and draining lymph nodes found multiple genes activated in old animals only after injury that were primarily related to IL17 signaling. Local inhibition of age-related type 3 immune activation using IL17-neutralizing antibodies restored therapeutic response to ECM and promoted muscle repair in older animals through increased recruitment of IL4 producing immune cells and regenerating muscle fibers. Altogether, innate and adaptive immune changes that occur with aging, in combination with dysregulated stromal communication, contribute to an impaired response to tissue injury which can be overcome with combination immunotherapy. 
Aging is associated with decreased tissue function and a compromised response to tissue damage that leads to longer recovery and frequently dysfunctional tissue repair regardless of tissue type ${ }^{1-}$ ${ }^{3}$. Reduced healing capacity with increasing age was recognized as early as $1932^{4}$. The variability in time required for tissue repair and quality increases with age in both preclinical models and patients $^{5-7}$, consistent with the variability in biological signatures of aging. Multi-omic analyses of cellular and molecular profiles of organisms over lifespan implicate changes in gene expression, metabolism, DNA methylation and epigenetic factors in age-associated pathologies including impaired wound healing ${ }^{2,3}$. Recovering tissue repair capacity that is lost with aging represents a significant medical challenge.

The composition and phenotype of cells responding to tissue damage changes with age. In skin wounds, the number of fibroblasts responding to injury is greater in older mice and the fibroblasts have reduced phenotypic heterogeneity compared to the wounds in younger counterparts $^{8}$. In the case of muscle tissue, the number and activity of muscle stem cells decreases with age leading to sarcopenia and impaired muscle healing after injury ${ }^{9}$. However, the functionality of aged muscle stem cells can be restored ex vivo to recover healing capacity after re-injection in vivo, suggesting that endogenous repair capacity is retained but the aging tissue environment impedes repair ${ }^{9}$. Similarly, repair in the aging retina could be restored by targeting age-related epigenetic changes ${ }^{10}$, again suggesting that regeneration capacity remains with increasing age despite decreased cell numbers and inhibitory factors.

Regenerative medicine and tissue engineering approaches are designed to enhance repair and restore tissue function. While many patients needing regenerative medicine technologies are older, the influence of age-related physiological changes on regenerative medicine therapeutic responses remains unexplored. In fact, age-related changes may be, in part, related to the disappointing clinical translation and efficacy of tissue engineering technologies and should be considered in their design. Classical regenerative medicine strategies utilize stem cells, growth factors and biomaterials alone or in combination to promote tissue development ${ }^{11}$. More recently, the role of the immune system in tissue repair is being recognized as a central factor in determining healing outcomes leading to the introduction of immunomodulation as a new therapeutic modality in regenerative medicine technology design. However, there are also numerous age-related changes that occur in the immune system, termed inflammaging, that may impede a regenerative therapeutic response ${ }^{12}$. Age-related immune changes have been primarily studied in the context of 
infectious disease, chronic inflammatory conditions, vaccine efficacy and more recently cancer immunotherapy efficacy but may also negatively impact the response to tissue damage and regenerative immunotherapies ${ }^{13}$. For example, $\mathrm{T}$ cell numbers decrease with aging and there is a myeloid shift in the bone marrow ${ }^{14,15}$. Furthermore, there are composition changes in the $\mathrm{T}$ cell compartment with aging that include increased $\mathrm{CD} 8^{+} \mathrm{T}$ cells, reduced naïve $\mathrm{CD} 4^{+} \mathrm{T}$ cells, and increased effector $\mathrm{CD}^{+}{ }^{+} \mathrm{T}$ cells which altogether may compromise tissue development ${ }^{14}$. Here, we investigated how immunological changes associated with aging impact the response to muscle injury and limit the regenerative capacity of a therapeutic biological scaffold. Targeting ageassociated immunological changes that inhibit a regenerative response may enable recovery of a therapeutic response and restoration of tissue repair capacity in older organisms. 


\section{Aging alters immune and stromal response to regenerative materials}

To evaluate the impact of aging on the response to a regenerative medicine therapy, we first characterized the response to an extracellular matrix (ECM) biomaterial in a muscle wound in young ( 6 week) and old (72 week) mice (Fig. 1a). ECM biomaterials, which can be derived from the matrix of different porcine and human tissue types ${ }^{16}$, are used for tissue repair in multiple clinical indications ${ }^{17,18}$ and are more easily delivered than cells and growth factors ${ }^{19-21}$. In this study, we utilized clinically available ${ }^{22}$ ECM from porcine small intestinal submucosa (SIS) as an example ECM material to model age-related differences in response. Muscle repair requires a type 2 adaptive and innate immune response along with IL4 signaling ${ }^{23,24}$, with eosinophils serving as a central producer of IL4. Application of an ECM biomaterial in a muscle injury increases IL4 expression and promotes repair in part by increasing recruitment of eosinophils and $\mathrm{CD} 4^{+} \mathrm{T}$ helper (Th) 2 cells $\mathrm{s}^{25}$.

Our first goal was to profile the local cell populations in the muscle tissue after injury and regenerative ECM treatment in young and old mice. Using multiparametric spectral flow cytometry (Extended Data Fig. 1), we found injury and ECM treatment resulted in distinct changes in immune and stromal cell responses in an aging tissue environment (Fig. 1b-c and Extended Data Fig. 2-4). A robust cellular response to a volumetric muscle loss (VML) injury and implant occurred in both young and aged animals but there were significant differences in the composition of the immune and stromal compartments (Extended Data Fig. 3-4). Specifically, the number and percentage of eosinophils in muscle tissue significantly increased in young animals with injury and ECM treatment compared to no injury controls (Fig. 1c and Extended Data Fig. 3-4). While the eosinophil counts and percentage also increased in the muscle injury of aged mice with ECM treatment, their percentage as a total of all $\mathrm{CD}_{11} \mathrm{~b}^{+}$immune cells was significantly lower compared to young counterparts (Fig. 1c).

The adaptive $\mathrm{T}$ cell immune response to muscle injury and ECM treatment also significantly changed with age with more CD8 $\mathrm{T}$ cells responding to injury in the aged animals compared to the CD4 and natural killer T (NKT) cell response in the young (Fig. 1c). Introduction of the ECM biomaterial further magnified the differences in $\mathrm{T}$ cell response producing a significant increase in CD4 and NKT cells in young mice and further increasing the CD8 T cells in aged mice (Extended Data Fig. 3). The significant increase in CD4 $\mathrm{T}$ cell numbers in young animals combined with the surge of CD8 $\mathrm{T}$ cells in the aged animals after ECM treatment resulted in a 
dramatic difference in the CD4 to CD8 ratio between young and aged mice that likely impacts the IL4 production required for muscle repair.

Additional changes in the injury and ECM response with aging occurred in the B cell and stromal cell populations. Before injury, the percentage of B cells was higher in aged muscle tissue (Extended Data Fig. 4). After injury and migration of immune cells to the damaged tissue, the percentage of B cells was low in all groups, however, ECM treatment significantly increased the number of B cells, specifically the mature $\mathrm{CD} 19^{+} \mathrm{B} 220^{+}$cells, only in the aged animals (Extended Data Fig. 3). Finally, the CD45' population, including fibroblasts, endothelial and other stromal cells, significantly increased in number after treatment with ECM in aged animals compared to young animals ( $p<0.0057$; Extended Data Fig. 3).

\section{Single cell analysis reveals changes in fibroblast signatures and myeloid antigen presentation after regenerative therapy in an aging environment}

To further identify age-related signatures of injury and therapeutic response to a regenerative biomaterial therapy, we performed single cell RNA sequencing (scRNA-seq) on CD45 ${ }^{+}$-enriched cells isolated from the muscle injuries with or without ECM treatment (Fig. 1a). We found 15 distinct cell clusters (Fig. 1d and Extended Data Fig. 5-7) that included myeloid cells, T cells, granulocytes, fibroblasts, endothelial/pericytes, and myogenic cells. We observed two different macrophage subtypes containing Mrc1 macrophage (Mrc1 Mc; Mrcl ${ }^{\text {hi }} C c l 8^{\text {hi }}$ ) and $\operatorname{Arg} 1$ macrophage (Arg1 Mc; $\operatorname{Arg} 1^{\mathrm{hi}} \mathrm{Mmp} 12^{\mathrm{hi}}$ ) and detected multiple clusters in the fibroblast population consisting of generic fibroblast (Gen Fib; Collal ${ }^{\text {hi }}$ Col3al ${ }^{\text {hi }}$ ), Mgp fibroblast (Mgp Fib;

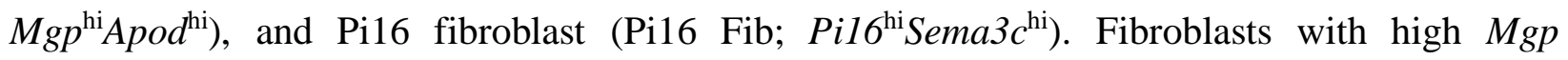
expression are typically defined as reticular fibroblasts ${ }^{26-29}$, and Pi16 is known to participate in regulating leukocyte infiltration and activation of the endothelial barrier ${ }^{30}$. Additionally, we identified generic myeloid cell (Gen Myl; Adgre $1^{\text {lo }} C c r 2^{\text {hi }} C d 74^{\text {hi }}$ ), granulocyte type 1 (Gr-1;

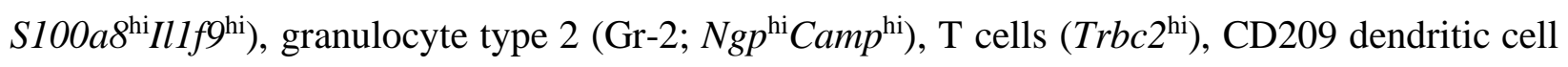
(CD209 DC; $C d 209 a^{\mathrm{hi}}$ ), a combination of endothelial cells and pericytes (Endo/Peri; $F a b p^{\text {hi }} \operatorname{Rgs}^{\mathrm{hi}}$ ), and myogenic cells (Myo; $\operatorname{Actal}^{\mathrm{hi}} M y h 4^{\mathrm{hi}}$ ).

There were changes in cell clusters from ECM-treated muscle injuries that were unique to young or aged animals (Extended Data Fig. 8). Granulocyte cluster Gr-2, enriched with Camp expression, increased after ECM treatment only in young animals. Camp is associated with early 
transcriptional states of neutrophils ${ }^{31,32}$ and their cytokine and chemokine profile induces migration of eosinophils ${ }^{33,34}$, supporting the increased eosinophil migration in young animals found by flow cytometry. The myogenic cell cluster Myo also increased with ECM treatment only in young animals, further suggesting that ECM increased myogenic activity primarily in young mice. On the other hand, the $C D 209^{\text {hi }}$ dendritic cell cluster increased with ECM only in aged mice. Cytokines from $C D 209^{\text {hi }}$ DCs are known to promote Th17 phenotypes in T cells ${ }^{35-38}$. Other clusters including Gr-1, Gen Myl, Mgp fib, and Endo/Peri changed with ECM treatment in a similar manner in both young and aged animals.

Next, we utilized a Bayesian non-negative matrix factorization (NMF) algorithm termed coordinate gene activity in pattern sets (CoGAPS) to capture additional cellular processes from the single cell dataset beyond changes in cell clusters ${ }^{39}$ (Fig. 1e). NMF is an alternative method to infer expression patterns that can span multiple clusters, reflective of biological processes ${ }^{40}$, with the Bayesian framework of CoGAPS having additional sparsity constraints ideal for scRNA-seq analysis. The CoGAPS NMF analysis found gene signatures of collagen production and assembly that are dominant in the muscle injury of aged animals (Extended Data Fig. 9). Gene signatures associated with collagen markers, such as Collal and Colla2, are enriched in fibroblasts from aged mice while the trend among the treatment groups was similar between young and aged animals (Fig. 1e, upper panel). Furthermore, a set of genes related to collagen matrix assembly, including Dcn, Fos, and Egrl ${ }^{41-43}$, is also more prominent in aged fibroblasts. ECM treatment further increased the expression of these collagen-associated genes in older animals compared to young animals, suggesting that injury and ECM treatment promoted collagen matrix formation primarily in the aging environment. When overlaid with the cell clustering dataset, the gene patterns were closely associated with specific fibroblast clusters: generic collagen genes with Gen Fib and collagen matrix assembly gene sets with Mgp Fib (Fig. 1e, upper panel circled with line). The majority of other gene signature patterns in the fibroblast populations are primarily present in the old animals (Extended Data Fig. 9).

CoGAPS analysis also highlighted gene profiles that were dominant in young animals in the myeloid and macrophage cells (Fig. 1e, lower panel and Extended Data Fig. 10). Genes associated with activated (Lgals3, Gpxl, Vim) $)^{44,45}$ or regulatory (Apoe, Clqb, Fcerlg) $)^{46,47}$ myeloid cells were all highly expressed in young animals compared to aged animals. While the treatment with ECM further increased the expression MHCII-associated genes $(C d 74, H 2-A a, H 2-E b 1)$ in 
both young and aged animals, their gene signatures were much more distinct within the young. When overlaid with cell clustering information, we found an MHCII-associated gene pattern predominant in the Gen Myl cluster that expressed H2-E, H2-A, and H2-DM (Extended Data Fig. 7), which increased with ECM treatment (Extended Data Fig. 8). The majority of additional gene patterns in myeloid and macrophage cells are also dominant in the young mice (Extended Data

Fig. 10). This suggests that even though some cell clusters increased with ECM treatment in a similar manner between young and aged mice, they may have different gene signatures that impact functional outcomes.

Altogether, the flow cytometry and single cell analysis demonstrate that key immune populations involved in muscle repair and a regenerative therapeutic response, such as eosinophils and CD4 T cells, decrease with aging. Furthermore, aging increases pro-inflammatory cells such as CD8 T cells and increases fibrosis signatures in response to regenerative treatments while at the same time decreasing immune activity features in myeloid and macrophages cells relevant for tissue repair including antigen presentation and mobilization.

\section{Aged animals exhibit reduced cell-cell communication networks and impaired fibroblast interactions}

To further probe the differences in the young and aged tissue environment after trauma and biomaterial application, we applied Domino analysis to model cell-cell communication patterns using the data obtained from scRNA-seq (Fig. 2). Domino is a computational tool that identifies condition-specific intercellular signaling dynamics based on transcription factor (TF) activation, which is surmised based on regulon expression with SCENIC gene regulatory network analysis ${ }^{48}$, along with receptor $(\mathrm{R})$ and ligand $(\mathrm{L})$ expression independent of cluster identification ${ }^{49}$. Domino constructs a signaling network connecting TF-R-L which are specifically predicted to be active in the data set. In both young and old animals, a force directed diagram of the TF-R-L signaling network self-assembled into three gene modules enriched in fibroblast, antigen processing and immune-tissue clusters (Fig. 2a). Each module indicates signaling pathways with similarly enriched activation in specific cell types, and connections between the modules indicate signaling patterns which are active in more than one cell type.

The fibroblast module in aged mice demonstrated a limited connection with other modules, suggesting impaired communication between immune and stromal populations. The antigen- 
processing module, represented by macrophages, myeloid cells, dendritic cells and $\mathrm{T}$ cells, interacts with both the fibroblast and immune-tissue module in young mice muscle. In aged animals, however, the fibroblast module reduced to only one connection with antigen processing and no interactions with the immune module, consistent with the CoGAPS results that showed limited immune activity features in myeloid and macrophage cells from aged mice.

Lack of inter-modular signaling with aging became more distinguished when inter-cluster dynamics are analyzed (Fig. 2a, bottom panel). Clusters such as $S 100 a 8^{\text {hi }} I l 1 f 9^{\text {hi }}$ Gr-1, Endo/Peri, and $\mathrm{T}$ cells demonstrated active communication networks with other cell populations in young mice, however, their inter-cluster crosstalk diminished in old mice. While there was still some communication among cell clusters in aged animals, their signaling was much weaker compared to young animals. In particular, the lack of communication with the Endo/Peri cluster is likely relevant to muscle tissue regeneration because muscle stem cells and satellite cells are known to recruit endothelial cells to secrete growth factors such as IGF-1, HGF, bFGF, and VEGF to promote satellite cell growth, thereby affecting the degree of capillarization of the myofibers, angiogenesis and ultimately myogenesis ${ }^{50}$.

\section{Unique transcription factor signaling in the aging environment correlates with type 3 immune profile in muscle and lymph node}

We then compared the transcription factors and receptors active in the young and old networks to identify signaling components that may be responsible for impaired cell networks and impaired wound healing with age (Fig. 2b-d and Extended Data Fig. 11-13). We identified several receptors common in both young and old signaling networks that correlated with activation of different TFs depending on age (Fig. 2b and Extended Data Fig. 12). For example, we found transforming growth factor beta receptor $1(T g f b r l)$ in the immune-tissue module of young and aged animals correlated with Foxo3 or Batf activation in aged mice while it was associated with Stat5b signaling in young mice. Foxo3 and Batf are crucial for the differentiation and function of Th17 cells ${ }^{51,52}$. Foxo3, a key immune-modulating gene associated with human longevity, has recently been shown to play an important role in IL21 secretion ${ }^{53}$ that can suppress the function of Th2 cells $^{54}$ and controls the generation of Th17 cells ${ }^{55}$. Batf is one of the activator protein-1 (AP1) proteins that controls Th17 differentiation ${ }^{52}$. On the other hand, Stat5, correlated with Tgfbrl in young animals, is critical for Th2 differentiation and IL4 production ${ }^{56}$ that is relevant for muscle 
repair and inhibition of Th17 differentiation via IL-2 signaling ${ }^{57}$. Additionally, a signal transduction adaptor Tyrobp, which was expressed in antigen processing modules in both age groups, correlated with Batf3 activation in old mice, but Maf in young mice. Batf3 regulates Th2 cell function while inhibiting the differentiation of regulatory T cells ${ }^{58,59}$, while Maf, though crucial for Th17 differentiation, serves as a key Th2-specific TF that induces Il4 gene transcription and is also critical in $I l 10$ gene expression in myeloid cells ${ }^{60-63}$.

We used String network to examine protein-protein interactions between the age-specific transcription factors (Fig. 2c right panel and Extended Data Fig. 13). The resulting network highlighted the aged animals-specific transcription factors, Foxo3, Mef2c, Tcf7l2, Cebpa, Stat1, and Batf as integral to the unique aging immune transcriptional network. To understand the biological significance of the age-specific TFs, we then performed gene set enrichment (GSE) analysis using Enrichr ${ }^{64,65}$, and enriched gene ontology (GO) terms to classify the biological processes in which they function (Fig. 2d). The young-specific TFs were associated with pathways involved with regulation of red blood cell differentiation or cytokinesis, and more importantly, endothelial cell differentiation. On the other hand, aged animal-specific TFs were enriched for fat cell differentiation, Th17 differentiation and myeloid DC differentiation pathways. Signaling associated with Th17 differentiation is known to negatively regulate eosinophil recruitment and IL4 expression ${ }^{54,66,67}$, suggesting that this immunological skewing in the TFs with aging may be responsible for the impaired Th2 response to the regenerative ECM biomaterial in the old animals.

To validate the computationally predicted type 3 immunological skewing in aged animals, we analyzed the baseline proximal inguinal lymph node (iLN) properties before injury. We first compared the gene expression profile of iLNs from naïve young and aged mice using Nanostring analysis (Fig. 2e and Extended Data Fig. 14-15) and flow cytometry (Fig. 2f and Extended Data Fig. 16). Aged lymph nodes expressed lower levels of general $\mathrm{T}$ cell markers such as $C d 3 e$ and $C d 3 d$, consistent with the known decrease in T cell numbers with aging. However, the expression of NF- $\mathrm{kb} / \mathrm{TNF} \alpha-$, Fc receptor- or Th17-associated gene sets, all of which are potent inducers of Th17-mediated inflammation ${ }^{68}$, increased in the aged lymph nodes (Fig. 2e and Extended Data Fig. 14). Pathway scoring further suggested that aging promoted $\mathrm{T}$ cell activation and skewing in Th17 differentiation or Th17-biology related genes most dramatically (Fig. 2e and Extended Data Fig. 15). Flow cytometric analysis further supported a type 3-skewed immune environment in the aging lymph node with a significantly higher proportion of IL17-producing CD4 and $\gamma \delta$ T cells 
present in the aged iLN compared to the young (Fig. 2f and Extended Data Fig. 16). While both the Th1 (IFN $\gamma^{+}$CD4) and Th17 (IL17a $\left.\mathrm{a}^{+} \mathrm{CD} 4\right)$ cells increased with aging, the $\gamma \delta \mathrm{T}$ cells switched phenotype from type $1\left(\mathrm{IFN} \gamma^{+}\right)$to type $3\left(\mathrm{IL} 17^{+}\right)$. Collectively, these data suggest that the lymph nodes highlight the immune dysfunction with aging, and impaired cell networks in aged tissues may be influenced by the changes in the lymph nodes.

\section{Aging induces a type 3 immune response to injury and therapy with impaired regenerative}

\section{response}

Next, we analyzed more in depth the source of immune dysfunction after injury and ECM treatment in old animals (Fig. 3). Cytokine gene expression and protein profiles in the muscle tissue differed with age (Fig. 3b-c). Il4 gene expression, critical for muscle repair, increased in muscle tissue only in young animals after injury with further significant increases after ECM treatment (Fig. 3b). In aged animals, injury did not increase Il4 expression in muscle, and its level of gene expression after ECM treatment was significantly lower than the young animals with ECM. Instead, ECM treatment in aged mice increased the expression of $I l 17 f$ (Ill7 $a$ gene expression was not detected in the muscle tissue of young or aged mice). Other inflammatory genes, including If $n \gamma, I l 23 a, I l 6, I l 1 b$, along with $C d k n 2 a$ and S100a4, all increased similarly in both young and old mice with injury or ECM treatment (Extended Data Fig. 17).

To investigate the cellular source of local IL17 signature, we then used young and aged IL17A-IRES-GFP-KI (IL17A-GFP) mice and probed IL17A expressing immune cells in the muscle tissue. $\gamma \delta \mathrm{T}$ cells, CD4 T cells, and innate lymphoid cells (ILC) all expressed IL17A and increased in numbers after ECM treatment (Extended Data Fig. 18). However, only IL17A ${ }^{+} \gamma \delta \mathrm{T}$ cell percentages significantly increased in aged animals with ECM treatment compared to injury alone (Fig. 3c). Additionally, the percentage of IL17A+ CD4 T cells increased significantly in aged animals with ECM treatment compared to no surgery. The percentage of ILCs expressing IL17A was similar among all groups except for naïve aged muscle tissue where there were significantly higher baseline levels, albeit in small cell numbers without injury or ECM treatment.

To determine the functional impact of the different immune environments in the young and aged animals on the ECM response and tissue repair, we evaluated the tissue structure histologically 1 week after injury and treatment (Fig. 3d and Extended Data Fig. 19). There was significant cell infiltration after injury in both young and aged mice, and ECM treatment further 
increased cellular infiltration and the presence of collagen as visualized by Masson's Trichrome staining. Consistent with the GSE analysis that demonstrated age-associated TFs were enriched for fat cell differentiation (Fig. 2d), there was excessive adipose tissue in the muscle of aged animals compared to the younger counterparts, which increased further with ECM treatment.

\section{Injury and ECM therapy induce a unique immune signature in the lymph nodes and blood of aged mice}

We then assessed the regional and systemic response to injury and ECM treatment in the lymph nodes and blood from young and aged mice (Fig. 3e-i). First, we observed visible differences in the size of the draining (inguinal) LNs with the young LNs appearing larger before and after injury and increasing further in size with ECM treatment compared to the aged animals (Fig. 3e). Quantification of iLN volume using caliper measurements confirmed a significant increase in size in the young animals with ECM treatment. The LN size correlated with total cell numbers in iLN with injury and ECM treatment significantly increasing the number of $\mathrm{CD}^{+} 5^{+}$cells in young animals, while only ECM treatment significant increased cell numbers in the aged animals albeit to a level significantly lower than young animals (Extended Data Fig. 20). In contrast to the lymph node, muscle tissue did not demonstrate significant differences in total CD $45^{+}$cell number between young and aged animals after ECM treatment (Extended Data Fig. 3), suggesting that the lymph node highlights the age-associated immune and regenerative dysfunction that may be responsible for poor muscle regeneration with age.

Gene expression for canonical cytokines in the lymph node correlated with the immune profiles found in the muscle tissue. ECM treatment significantly increased Il4 expression in young iLNs only (Fig. 3f) and there were minimal changes in Ifny expression. Strikingly, both Ill7a and Ill7f significantly increased only in aged animals after injury and ECM treatment. We further analyzed the expression of genes that correlate with Th17 skewing, as supported by String network; Mmp3, Mmp9, Cxcll, Ccl20, and $C x c l 5^{69}$. Expression of these genes occurred at low levels in all conditions in naïve young and aged animals but significantly increased only after injury and ECM treatment in aged mice (Fig. 3g), suggesting that injury triggered a type 3 immune response in an aging tissue environment. Multiparametric flow cytometry analysis of the cells from the iLN further confirmed the trend with increasing numbers and percentages of IL $17 \mathrm{~A}^{+} \gamma \delta$ and CD4 T cells after ECM treatment only in aged mice (Fig. 3h and Extended Data Fig. 21). In 
addition, B cells also exhibited age-specific changes after injury and ECM treatment. Similar to the increased number of B cells in aging muscle tissue (Extended Data Fig. 3), iLN from old animals had significantly higher percentages of B cells after injury and treatment, further skewing T to B cell ratio with age (Extended Data Fig. 20).

To further assess the systemic immune changes with aging, we analyzed cytokines in serum using proteome profiler (Fig. 3i and Extended Data Fig. 22). In addition to increased IL17 in the serum of aged mice, there was also increased levels of the B cell chemoattractant factor CXCL13, suggesting that the increased B cell signatures in aged muscle and lymph nodes also extended systemically. CXCL13 significantly increased in blood after injury in old mice, further supporting that injury promotes a B cell response solely in old animals. Additionally, IL1A, a potent inducer of IL17 from T cells ${ }^{70}$, and CXCL1, one of IL17-induced chemokines, also increased in serum from the aged mice. IL23, a protein that can expand Th17 cells ${ }^{71}$, increased in response to injury only in old mice. Collectively, these data show that injury and ECM treatment in aged animals triggered a local and systemic type 3-mediated immune response that may be responsible for the decreased regenerative and therapeutic response with aging.

\section{Inhibition of IL17 rejuvenates type 2 response after muscle injury in aged animals}

Since IL17 expression in the muscle tissue and lymph nodes increased with aging, we investigated if IL17 neutralizing antibodies ( $\alpha$ IL17) could restore IL4 expression and tissue repair. We first evaluated the dose and timing for delivery (Extended Data Fig. 23). A minimum of three injections was required to reduce inflammatory markers in the tissue after injury in aged mice. However, initiating $\alpha \mathrm{IL} 17$ injections at the time of injury stunted infiltration of immune (CD45 $\left.{ }^{+}\right)$ cells including $\mathrm{IL}^{+}$cells that are critical for tissue repair, suggesting the importance of acute IL17 during wound healing to attract the immune effectors and initiate tissue regeneration (Extended Data Fig. 23b). We then tested initiation of $\alpha$ IL17 injections one week after injury to allow immune infiltration before treatment (Fig. 4a). Using aged I1 ${ }^{\text {tm } 1 \text { Lky }}$ mice (4Get), which have a fluorescent reporter for IL4 expression, we found that both $\alpha$ IL17A and $\alpha$ IL17F treatment one week after injury and ECM treatment significantly increased the number of IL4 ${ }^{+}$eosinophils and CD4 T cells three weeks after injury. 


\section{Combination therapy of pro-regenerative ECM and $\alpha \mathrm{IL17}$ rejuvenates muscle repair in aged mice}

Since the aged animals demonstrated a type 3 immune profile, which is associated with fibrosis ${ }^{72,73}$ and negatively regulates the IL4 expression ${ }^{54,66}$, we tested a combination therapy approach with pro-regenerative ECM and aIL17 (Fig. 4b). Muscle wounds in aged animals received ECM treatment at the time of surgery and local $\alpha$ IL17A or $\alpha$ IL17F one week after injury. Six weeks after injury in aged 4Get mice, the antibody treatment after ECM implantation increased CD $45^{+}$cell numbers (Extended Data Fig. 24), however, only the $\alpha$ IL17A treated animals had a significantly higher number and percentage of $\mathrm{IL}^{+} \mathrm{CD} 45^{+}$immune cells and number of $\mathrm{IL} 4^{+} \mathrm{CD} 4^{+} \mathrm{T}$ cells compared to the $\alpha$ IL17F or ECM only groups, albeit in small numbers at this later time point. Three weeks after combination therapy, we observed a significant decrease in $\gamma \delta \mathrm{T}$ cell numbers in mice treated with $\alpha$ IL17A or $\alpha$ IL17F using multi-spectral flow cytometry (Extended Data Fig. 25).

We then further explored the therapeutic response to ECM- $\alpha$ IL17A combination in the muscle wound and tissue repair. Six weeks after injury, muscle tissue from aged C57BL/6J mice treated with ECM and $\alpha$ IL17A expressed significantly lower levels of numerous fibrosis- or adipose-associated genes that CoGAPS analysis identified as increasing in aged animals (Fig. 4c top left). Specifically, collagen type III a1 (Col3al) and collagen type V a1 (Col5al) decreased significantly with $\alpha$ IL17A treatment. Additionally, adipose-associated genes, such as Ppar $\gamma$, Fabp4, and Adipoq all dramatically decreased with $\alpha$ IL17A treatment. Similarly, other fibrosisrelated genes, such as Fap and Pdgfa ${ }^{74}$, decreased with $\alpha$ IL17A or $\alpha$ IL17A/ $\alpha$ IL17F (Extended Data Fig. 26). Expression of the inflammatory gene Mmpl3 significantly decreased with any combination of IL17 antibody treatments, however, RAR-related orphan receptor type c (Rorc), which is critical in the differentiation of Th17 15 , was only downregulated with a combination of $\alpha I L 17 A / \alpha I L 17 F$ treatment. Differences in gene expression profile between the $\alpha$ IL17A and $\alpha \mathrm{IL17F}$-treated groups suggest that there may be a distinction between IL17a and IL17f signaling pathways.

Histological evaluation of the muscle defect in aged animals treated with combination therapy supported the immunological and gene expression results with increased repair and reduced fibrosis depending on the form of IL17 neutralization (Fig. 4c and Extended Data Fig. 27). Aged mice treated with ECM alone demonstrated collagen deposition and fibrosis that 
decreased with $\alpha$ IL17A or combination $\alpha$ IL17A/ $\alpha$ IL17F treatment as visualized by Masson's trichrome. Adipose deposition in the repair tissue also decreased with ECM- $\alpha$ IL17A combination treatment as visualized in the histology, supporting gene expression results. Additionally, a combination treatment induced nuclear repositioning from the periphery to the center of muscle cells (Fig. 4d), a characteristic of repairing muscle tissue that did not occur in animals treated with injury or ECM alone. Nuclear positioning is critical in muscle fiber function, and it was shown that myofibers that are regenerated several weeks after muscle damage can be characterized by centrally localized nuclei ${ }^{76,77}$. Quantification of muscle cells with centrally-located nuclei demonstrated that ECM- $\alpha$ IL17A combination treatment significantly increased the percentage of regenerating muscle fibers compared to the injury or ECM alone groups (Fig. 4d, right). Altogether, we demonstrate that aging significantly alters the immune and stromal response to muscle injury and therapeutic biomaterials in the local tissue, regional lymph nodes and systemically in blood. These altered responses, characterized by increased IL17, reduced IL4 and excess fibrosis and adipogenesis, can be mitigated with a combination therapy of a proregenerative ECM material and an IL17 antibody to promote the repair capacity in aging tissue.

\section{Discussion}

Aging is associated with numerous chronic diseases and increased incidence of cancer ${ }^{78}$. However, healthy aging, even without overt disease, results in longer recovery times from tissue injury. Changes in cellular composition with aging may be in part responsible for reduced healing capacity including decreased endogenous stem cell numbers and activity, in addition to reduced fibroblast heterogeneity ${ }^{8,9}$. More recently, epigenetic changes have also been implicated in age-associated repair dysfunction ${ }^{79}$. However, aging also impacts the immune system, promoting chronic inflammation and multiple immune system abnormalities that will modulate the injury response. Tissue injury mobilizes the immune system and uncovers additional age-associated dysfunctions that are otherwise not apparent. As regenerative medicine strategies are moving to target the immune system, understanding these age-associated immune changes will be critical to develop therapies that are relevant to the older patient populations that are more likely to suffer from delayed or inadequate tissue repair.

While multiple approaches are used in regenerative medicine therapies, we chose ECM biomaterials to evaluate in an aging environment because of their clinical use ${ }^{16}$. Few regenerative 
medicine therapies have reached standard clinical practice and now even the mechanism of longstudied stem cell therapies is being reconsidered, as non-viable cells appear to promote similar therapeutic responses as viable cells ${ }^{80}$. ECM biomaterials derived from allograft and porcine sources are approved for wound healing and reconstructive surgery applications, orthopedic, and ophthalmologic indications ${ }^{16,81,82}$. ECM materials contain a complex mixture of proteins, proteoglycans, and even matrix-bound vesicles that likely all contribute to damage signals and other as yet determined factors that mobilize multiple immune and stromal cell types to promote tissue repair. It is likely that other regenerative medicine approaches, cellular, growth factors or small molecules, will exhibit similar reduced efficacy with aging that we found using ECM.

With the numerous cellular, molecular, and epigenetic changes that occur with aging, it is expected that combination therapies may be needed to shift regenerative responses. Multiple cell types respond to the complex ECM and multiple immune cells produce IL17 or have receptors to IL17-associated signals. The cell communication analysis by Domino uncovered reduced cellular interactions with aging, particularly with the stromal fibroblasts, and reduced antigen presentation and myeloid activity highlighting multiple dysfunctions. These interactomes provided candidates for elucidating the unique changes that only occurred after injury in the aged organism. Many of these were related to IL17 signaling. The cytokine IL17 is a component of the host defense against extracellular pathogens ${ }^{55,66}$, but is also associated with fibrosis and fibrotic disease ${ }^{72,73}$, suggesting a common mechanism of "walling off" uncontrolled pathogens and maintaining barrier surfaces and microbiome balance. Age-associated commensal dysbiosis may contribute to the excess IL17 in addition to senescence-induced immunomodulation that promotes IL17 $7^{83}$. IL17 is important for the recruitment of effector immune cells for wound repair and host defense, yet its chronic development with aging can further induce carcinogenesis, fibrosis, and inappropriate immune response. Overall, local immune and stromal modulation in the form of combination therapies may be needed in an aging environment to address the multiple dysfunctional trauma responses that occur with aging.

\section{Acknowledgements}

This work was funded in part by the National Institutes of Health (NIH) Pioneer Award DP1AR076959 (J.H.E.), Bloomberg Kimmel Institute, Morton Goldberg Professorship (J.H.E.), the Department of Defense, R01EB028796 (J.H.E.), and R01AG063801(J.H.E.). A.R. and D.R.M. 
were funded through NSF GRFP DGE-1746891. We thank F. Housseau for providing the IL17AGFP mice. Schematic illustrations created with BioRender.com.

\section{Author contributions}

J.H., and J.H.E. conceptualized the study, drafted, and edited the figures and manuscript. J.H., J.H.E., and D.M.P. contributed to experimental design and interpretation of the results. J.H. performed animal surgery model and analyzed experimental results (flow cytometry, qRT-PCR, proteome profiler, histology, and analysis). C.C. wrote software and assisted in single cell RNA sequencing analysis and computational methodology. A.R., J.C.M., and D.R.M. assisted with animal breeding and spectral flow cytometry analysis. H.H.N. contributed to conducting histological evaluation. E.J.F. advised in the bioinformatics analysis. F.H., S.G., and A.J.T. assisted with generalized gene expression interpretation and flow cytometry results. E.M.M. assisted with Nanostring analysis.

\section{Competing interests}

J.H.E. holds equity in Unity Biotechnology and Aegeria Soft Tissue and is an advisor for Tessera Therapeutics. D.M.P is consultant at Aduro Biotech, Amgen, Astra Zeneca, Bayer, Compugen, DNAtrix, Dynavax Technologies Corporation, Ervaxx, FLX Bio, Immunomic, Janssen, Merck, and Rock Springs Capital. D.M.P. holds equity in Aduro Biotech, DNAtrix, Ervaxx, Five Prime therapeutics, Immunomic, Potenza, Trieza Therapeutics. D.M.P is a member of the scientific advisory board for Bristol Myers Squibb, Camden Nexus II, Five Prime Therapeutics, and WindMil. D.M.P. is a member of board of directors in Dracen Pharmaceuticals. C.C. is the founder and owner of C M Cherry Consulting, LLC. E.J.F. is a member of the scientific advisory board for Viosera Therapeutics. 


\section{References}

1 Kirkwood, T. B. Understanding the odd science of aging. Cell 120, 437-447, doi:10.1016/j.cell.2005.01.027 (2005).

2 Pisco, A. O. et al. A single cell transcriptomic atlas characterizes aging tissues in the mouse. BioRxiv, 661728 (2019).

3 van Deursen, J. M. The role of senescent cells in ageing. Nature 509, 439-446, doi:10.1038/nature13193 (2014).

4 Du Noüy, P. L. Cicatrization of wounds: III. The relation between the age of the patient, the area of the wound, and the index of cicatrization. J Exp Med 24, 461-470 (1916).

5 Gosain, A. \& DiPietro, L. A. Aging and wound healing. World J Surg 28, 321-326, doi:10.1007/s00268-003-7397-6 (2004).

6 Gould, L. et al. Chronic wound repair and healing in older adults: current status and future research. J Am Geriatr Soc 63, 427-438, doi:10.1111/jgs.13332 (2015).

7 Emery, C. F., Kiecolt-Glaser, J. K., Glaser, R., Malarkey, W. B. \& Frid, D. J. Exercise accelerates wound healing among healthy older adults: a preliminary investigation. $J$ Gerontol A Biol Sci Med Sci 60, 1432-1436, doi:10.1093/gerona/60.11.1432 (2005).

8 Mahmoudi, S. et al. Heterogeneity in old fibroblasts is linked to variability in reprogramming and wound healing. Nature 574, 553-558 (2019).

9 Cosgrove, B. D. et al. Rejuvenation of the muscle stem cell population restores strength to injured aged muscles. Nat Med 20, 255-264, doi:10.1038/nm.3464 (2014).

10 Pennington, K. L. \& DeAngelis, M. M. Epigenetic Mechanisms of the Aging Human Retina. J Exp Neurosci 9, 51-79, doi:10.4137/JEN.S25513 (2015).

11 Howard, D., Buttery, L. D., Shakesheff, K. M. \& Roberts, S. J. Tissue engineering: strategies, stem cells and scaffolds. J Anat 213, 66-72, doi:10.1111/j.14697580.2008.00878.x (2008).

12 Franceschi, C., Garagnani, P., Parini, P., Giuliani, C. \& Santoro, A. Inflammaging: a new immune-metabolic viewpoint for age-related diseases. Nat Rev Endocrinol 14, 576-590, doi:10.1038/s41574-018-0059-4 (2018).

13 Castle, S. C. Clinical relevance of age-related immune dysfunction. Clin Infect Dis 31, 578585, doi:10.1086/313947 (2000).

14 Nikolich-Zugich, J. Ageing and life-long maintenance of T-cell subsets in the face of latent persistent infections. Nat Rev Immunol 8, 512-522, doi:10.1038/nri2318 (2008).

15 Pang, W. W. et al. Human bone marrow hematopoietic stem cells are increased in frequency and myeloid-biased with age. Proc Natl Acad Sci U S A 108, 20012-20017, doi:10.1073/pnas.1116110108 (2011).

16 Badylak, S. F., Freytes, D. O. \& Gilbert, T. W. Extracellular matrix as a biological scaffold material: Structure and function. Acta Biomater 5, 1-13, doi:10.1016/j.actbio.2008.09.013 (2009).

17 Brown, B. N. et al. Macrophage phenotype as a predictor of constructive remodeling following the implantation of biologically derived surgical mesh materials. Acta Biomater 8, 978-987, doi:10.1016/j.actbio.2011.11.031 (2012).

18 Mase, V. J., Jr. et al. Clinical application of an acellular biologic scaffold for surgical repair of a large, traumatic quadriceps femoris muscle defect. Orthopedics 33, 511, doi:10.3928/01477447-20100526-24 (2010). 
19 Lee, K., Silva, E. A. \& Mooney, D. J. Growth factor delivery-based tissue engineering: general approaches and a review of recent developments. J R Soc Interface 8, 153-170, doi:10.1098/rsif.2010.0223 (2011).

20 Teodori, L. et al. Native extracellular matrix: a new scaffolding platform for repair of damaged muscle. Front Physiol 5, 218, doi:10.3389/fphys.2014.00218 (2014).

21 Wang, Z. et al. Novel biomaterial strategies for controlled growth factor delivery for biomedical applications. NPG Asia Mater 9, e435-e435 (2017).

22 Mosala Nezhad, Z. et al. Small intestinal submucosa extracellular matrix (CorMatrix(R)) in cardiovascular surgery: a systematic review. Interact Cardiovasc Thorac Surg 22, 839850, doi:10.1093/icvts/ivw020 (2016).

23 Gieseck III, R. L., Wilson, M. S. \& Wynn, T. A. Type 2 immunity in tissue repair and fibrosis. Nat Rev Immunol 18, 62 (2018).

24 Heredia, J. E. et al. Type 2 innate signals stimulate fibro/adipogenic progenitors to facilitate muscle regeneration. Cell 153, 376-388, doi:10.1016/j.cell.2013.02.053 (2013).

25 Sadtler, K. et al. Developing a pro-regenerative biomaterial scaffold microenvironment requires T helper 2 cells. Science 352, 366-370, doi:10.1126/science.aad9272 (2016).

26 Janson, D. G., Saintigny, G., van Adrichem, A., Mahe, C. \& El Ghalbzouri, A. Different gene expression patterns in human papillary and reticular fibroblasts. $J$ Invest Dermatol 132, 2565-2572, doi:10.1038/jid.2012.192 (2012).

27 Korosec, A. et al. Lineage Identity and Location within the Dermis Determine the Function of Papillary and Reticular Fibroblasts in Human Skin. J Invest Dermatol 139, 342-351, doi:10.1016/j.jid.2018.07.033 (2019).

28 Haydont, V., Neiveyans, V., Fortunel, N. O. \& Asselineau, D. Transcriptome profiling of human papillary and reticular fibroblasts from adult interfollicular dermis pinpoints the 'tissue skeleton' gene network as a component of skin chrono-ageing. Mech Ageing Dev 179, 60-77, doi:10.1016/j.mad.2019.01.003 (2019).

29 Sole-Boldo, L. et al. Single-cell transcriptomes of the human skin reveal age-related loss of fibroblast priming. Commun Biol 3, 188, doi:10.1038/s42003-020-0922-4 (2020).

30 Singhmar, P. et al. The fibroblast-derived protein PI16 controls neuropathic pain. Proc Natl Acad Sci U S A 117, 5463-5471, doi:10.1073/pnas.1913444117 (2020).

31 Calcagno, D. M. et al. SiglecF(HI) Marks Late-Stage Neutrophils of the Infarcted Heart: A Single-Cell Transcriptomic Analysis of Neutrophil Diversification. J Am Heart Assoc 10, e019019, doi:10.1161/JAHA.120.019019 (2021).

32 Xie, X. et al. Single-cell transcriptome profiling reveals neutrophil heterogeneity in homeostasis and infection. Nat Immunol 21, 1119-1133, doi:10.1038/s41590-020-0736-Z (2020).

33 Agier, J., Efenberger, M. \& Brzezinska-Blaszczyk, E. Cathelicidin impact on inflammatory cells. Cent Eur J Immunol 40, 225-235, doi:10.5114/ceji.2015.51359 (2015).

34 Zhang, X. J. et al. Distinctive structural hallmarks and biological activities of the multiple cathelicidin antimicrobial peptides in a primitive teleost fish. $J$ Immunol 194, 4974-4987, doi:10.4049/jimmunol.1500182 (2015).

35 Kalantari, P., Bunnell, S. C. \& Stadecker, M. J. The C-type Lectin Receptor-Driven, Th17 Cell-Mediated Severe Pathology in Schistosomiasis: Not All Immune Responses to Helminth Parasites Are Th2 Dominated. Front Immunol 10, 26, doi:10.3389/fimmu.2019.00026 (2019). 
36 Kalantari, P. et al. CD209a Synergizes with Dectin-2 and Mincle to Drive Severe Th17 Cell-Mediated Schistosome Egg-Induced Immunopathology. Cell Rep 22, 1288-1300, doi:10.1016/j.celrep.2018.01.001 (2018).

37 Ponichtera, H. E. et al. CD209a expression on dendritic cells is critical for the development of pathogenic Th17 cell responses in murine schistosomiasis. J Immunol 192, 4655-4665, doi:10.4049/jimmunol.1400121 (2014).

38 Wang, J. et al. A Positive Feedback Loop Between Th17 Cells and Dendritic Cells in Patients with Endplate Inflammation. Immunol Invest 48, 39-51, doi:10.1080/08820139.2018.1496097 (2019).

39 Stein-O'Brien, G. L. et al. Decomposing Cell Identity for Transfer Learning across Cellular Measurements, Platforms, Tissues, and Species. Cell Syst 8, 395-411 e398, doi:10.1016/j.cels.2019.04.004 (2019).

40 Stein-O'Brien, G. L. et al. Enter the Matrix: Factorization Uncovers Knowledge from Omics. Trends Genet 34, 790-805, doi:10.1016/j.tig.2018.07.003 (2018).

41 Zhang, W. et al. Decorin is a pivotal effector in the extracellular matrix and tumour microenvironment. Oncotarget 9, 5480-5491, doi:10.18632/oncotarget.23869 (2018).

42 Havis, E. \& Duprez, D. EGR1 Transcription Factor is a Multifaceted Regulator of Matrix Production in Tendons and Other Connective Tissues. Int J Mol Sci 21, doi:10.3390/ijms21051664 (2020).

43 Rosenfeldt, H., Lee, D. J. \& Grinnell, F. Increased c-fos mRNA expression by human fibroblasts contracting stressed collagen matrices. Mol Cell Biol 18, 2659-2667, doi:10.1128/MCB.18.5.2659 (1998).

44 Park, D. D. et al. Resident and elicited murine macrophages differ in expression of their glycomes and glycan-binding proteins. Cell Chem Biol 28, 567-582. e564 (2021).

45 Mor-Vaknin, N., Punturieri, A., Sitwala, K. \& Markovitz, D. M. Vimentin is secreted by activated macrophages. Nat Cell Biol 5, 59-63 (2003).

46 Poon, C. C. et al. Differential microglia and macrophage profiles in human IDH-mutant and-wild type glioblastoma. Oncotarget 10, 3129 (2019).

47 Gu, Z., Chhabra, A., Alard, P., Warner, D. \& Kosiewicz, M. Fc $\gamma R I$ is required for TGF $\beta 2-$ treated macrophage-induced tolerance. Immunobiology 218, 1200-1206 (2013).

48 Aibar, S. et al. SCENIC: single-cell regulatory network inference and clustering. Nat Methods 14, 1083-1086, doi:10.1038/nmeth.4463 (2017).

49 Cherry, C. et al. Intercellular signaling dynamics from a single cell atlas of the biomaterials response. bioRxiv (2020).

50 Yin, H., Price, F. \& Rudnicki, M. A. Satellite cells and the muscle stem cell niche. Physiol Rev 93, 23-67, doi:10.1152/physrev.00043.2011 (2013).

51 Bouzeyen, R., Haoues, M., Barbouche, M.-R., Singh, R. \& Essafi, M. FOXO3 transcription factor regulates IL-10 expression in Mycobacteria-infected macrophages, tuning their polarization and the subsequent adaptive immune response. Front Immunol 10, 2922 (2019).

52 Ciofani, M. et al. A validated regulatory network for Th17 cell specification. Cell 151, 289-303, doi:10.1016/j.cell.2012.09.016 (2012).

53 Qi, H. et al. Foxo3 Promotes the Differentiation and Function of Follicular Helper T Cells. Cell Rep 31, 107621, doi:10.1016/j.celrep.2020.107621 (2020). 
54 Lin, P. Y., Jen, H. Y., Chiang, B. L., Sheu, F. \& Chuang, Y. H. Interleukin-21 suppresses the differentiation and functions of $\mathrm{T}$ helper 2 cells. Immunology 144, 668-676, doi:10.1111/imm.12419 (2015).

55 Martinez, G. J., Nurieva, R. I., Yang, X. O. \& Dong, C. Regulation and function of proinflammatory TH17 cells. Ann $N \quad Y$ Acad Sci 1143, 188-211, doi:10.1196/annals.1443.021 (2008).

56 Zhu, J., Cote-Sierra, J., Guo, L. \& Paul, W. E. Stat5 activation plays a critical role in Th2 differentiation. Immunity 19, 739-748, doi:10.1016/s1074-7613(03)00292-9 (2003).

57 Laurence, A. et al. Interleukin-2 signaling via STAT5 constrains T helper 17 cell generation. Immunity 26, 371-381, doi:10.1016/j.immuni.2007.02.009 (2007).

58 Kuwahara, M. et al. Bach2-Batf interactions control Th2-type immune response by regulating the IL-4 amplification loop. Nat Commun 7, 12596, doi:10.1038/ncomms12596 (2016).

59 Lee, W., Kim, H. S., Hwang, S. S. \& Lee, G. R. The transcription factor Batf3 inhibits the differentiation of regulatory $\mathrm{T}$ cells in the periphery. Exp Mol Med 49, e393, doi:10.1038/emm.2017.157 (2017).

60 Ho, I.-C., Lo, D. \& Glimcher, L. H. c-maf promotes T helper cell type 2 (Th2) and attenuates Th1 differentiation by both interleukin 4-dependent and-independent mechanisms. J Exp Med 188, 1859-1866 (1998).

61 Kim, J. I., Ho, I.-C., Grusby, M. J. \& Glimcher, L. H. The transcription factor c-Maf controls the production of interleukin-4 but not other Th2 cytokines. Immunity 10, 745-751 (1999).

62 Homma, Y., Cao, S., Shi, X. \& Ma, X. The Th2 transcription factor c-Maf inhibits IL12 p35 gene expression in activated macrophages by targeting NF-kappaB nuclear translocation. J Interferon Cytokine Res 27, 799-808, doi:10.1089/jir.2007.0006 (2007).

63 Kaiko, G. E., Horvat, J. C., Beagley, K. W. \& Hansbro, P. M. Immunological decisionmaking: how does the immune system decide to mount a helper T-cell response? Immunology 123, 326-338 (2008).

64 Chen, E. Y. et al. Enrichr: interactive and collaborative HTML5 gene list enrichment analysis tool. BMC Bioinformatics 14, 128, doi:10.1186/1471-2105-14-128 (2013).

65 Kuleshov, M. V. et al. Enrichr: a comprehensive gene set enrichment analysis web server 2016 update. Nucleic Acids Res 44, W90-97, doi:10.1093/nar/gkw377 (2016).

66 Schmidt-Weber, C. B., Akdis, M. \& Akdis, C. A. TH17 cells in the big picture of immunology. J Allergy Clin Immunol 120, 247-254, doi:10.1016/j.jaci.2007.06.039 (2007).

67 Ohnmacht, C. et al. The microbiota regulates type 2 immunity through ROR $\gamma \mathrm{t}+\mathrm{T}$ cells. Science 349, 989-993 (2015).

68 Liu, T., Zhang, L., Joo, D. \& Sun, S.-C. NF-кB signaling in inflammation. Signal Transduct Target Ther 2, 1-9 (2017).

$69 \mathrm{Xu}, \mathrm{S} . \& \mathrm{Cao}, \mathrm{X}$. Interleukin-17 and its expanding biological functions. Cell Mol Immunol 7, 164-174, doi:10.1038/cmi.2010.21 (2010).

70 Sutton, C., Brereton, C., Keogh, B., Mills, K. H. \& Lavelle, E. C. A crucial role for interleukin (IL)-1 in the induction of IL-17-producing $\mathrm{T}$ cells that mediate autoimmune encephalomyelitis. J Exp Med 203, 1685-1691 (2006). 
71 Bunte, K. \& Beikler, T. Th17 Cells and the IL-23/IL-17 Axis in the Pathogenesis of Periodontitis and Immune-Mediated Inflammatory Diseases. Int J Mol Sci 20, 3394, doi:10.3390/ijms20143394 (2019).

72 Fabre, T. et al. Type 3 cytokines IL-17A and IL-22 drive TGF-beta-dependent liver fibrosis. Sci Immunol 3, doi:10.1126/sciimmunol.aar7754 (2018).

73 Tesmer, L. A., Lundy, S. K., Sarkar, S. \& Fox, D. A. Th17 cells in human disease. Immunol Rev 223, 87-113, doi:10.1111/j.1600-065X.2008.00628.x (2008).

74 Malecova, B. et al. Dynamics of cellular states of fibro-adipogenic progenitors during myogenesis and muscular dystrophy. Nat Commun 9, 3670, doi:10.1038/s41467-01806068-6 (2018).

75 Yang, X. O. et al. T helper 17 lineage differentiation is programmed by orphan nuclear receptors ROR $\alpha$ and ROR $\gamma$. Immunity 28, 29-39 (2008).

76 Folker, E. S. \& Baylies, M. K. Nuclear positioning in muscle development and disease. Front Physiol 4, 363, doi:10.3389/fphys.2013.00363 (2013).

77 Cadot, B., Gache, V. \& Gomes, E. R. Moving and positioning the nucleus in skeletal muscle-one step at a time. Nucleus 6, 373-381 (2015).

78 Prasad, S., Sung, B. \& Aggarwal, B. B. Age-associated chronic diseases require age-old medicine: role of chronic inflammation. Prev Med 54 Suppl, S29-37, doi:10.1016/j.ypmed.2011.11.011 (2012).

79 Pal, S. \& Tyler, J. K. Epigenetics and aging. Sci Adv 2, e1600584, doi:10.1126/sciadv.1600584 (2016).

80 Wagoner, Z. W. \& Zhao, W. Therapeutic implications of transplanted-cell death. Nat Biomed Eng 5, 379-384, doi:10.1038/s41551-021-00729-6 (2021).

81 Liu, L. et al. Evaluation of the biocompatibility and mechanical properties of xenogeneic (porcine) extracellular matrix (ECM) scaffold for pelvic reconstruction. Int Urogynecol J 22, 221-227, doi:10.1007/s00192-010-1288-9 (2011).

82 Chow, D. W. \& Westermeyer, H. D. Retrospective evaluation of corneal reconstruction using AC ell Vet ${ }^{\mathrm{TM}}$ alone in dogs and cats: 82 cases. Vet Ophthalmol 19, 357-366 (2016).

83 Faust, H. J. et al. IL-17 and immunologically induced senescence regulate response to injury in osteoarthritis. J Clin Invest 130, 5493-5507, doi:10.1172/JCI134091 (2020). 
a.

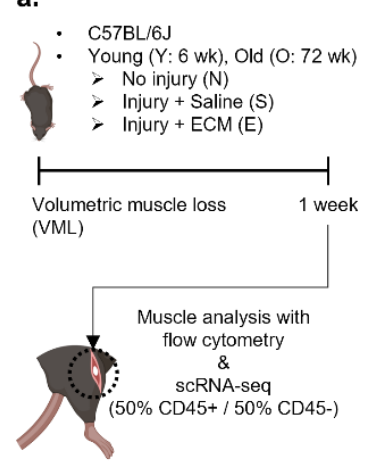

d.

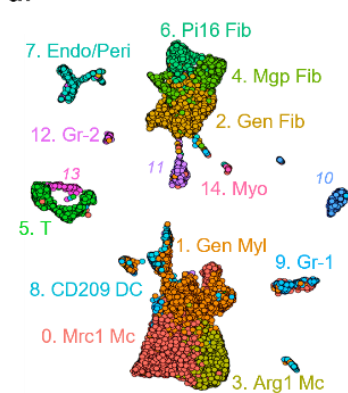

- 10: Hemoglobin (RBC) - 11: Stromal cell cyclin - 13: T cell cycling b.
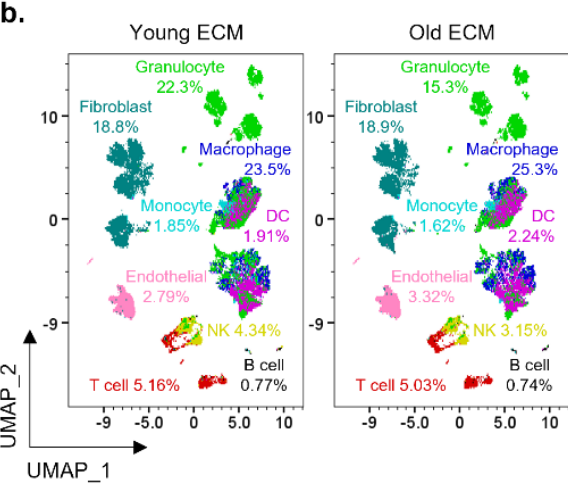

CD45+ cells

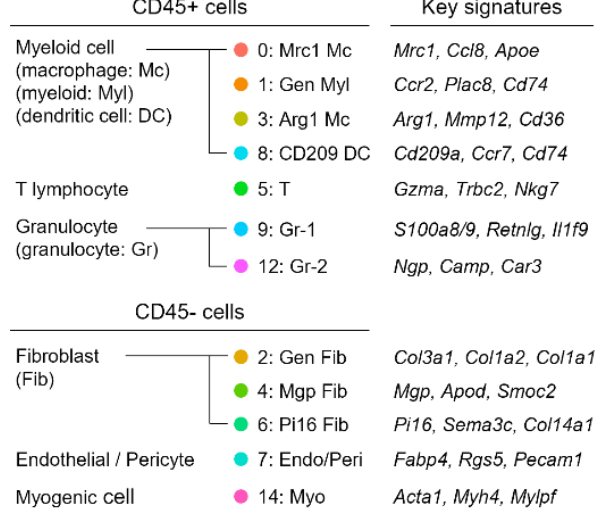

c.
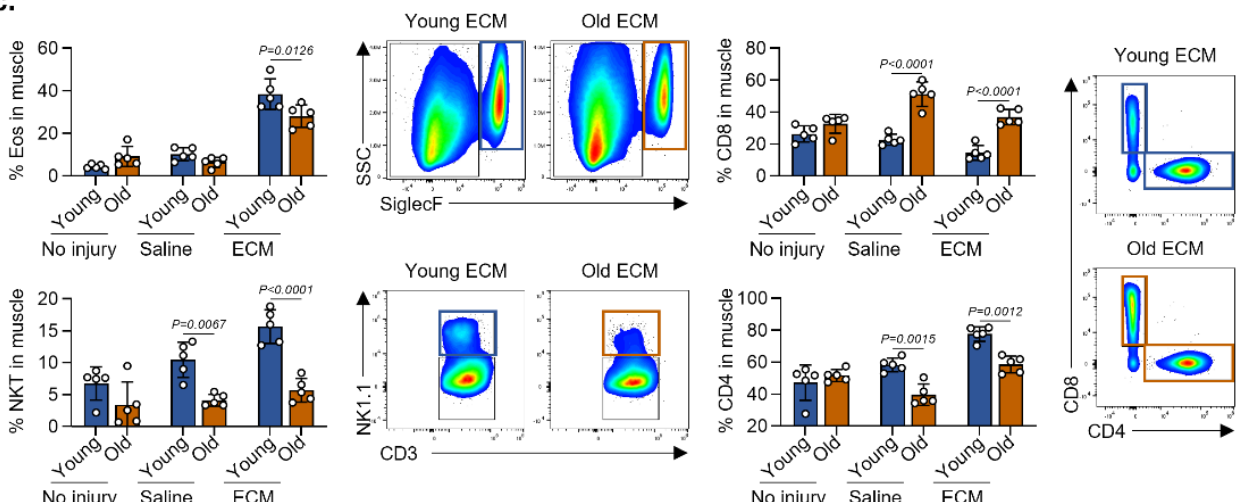

No inj
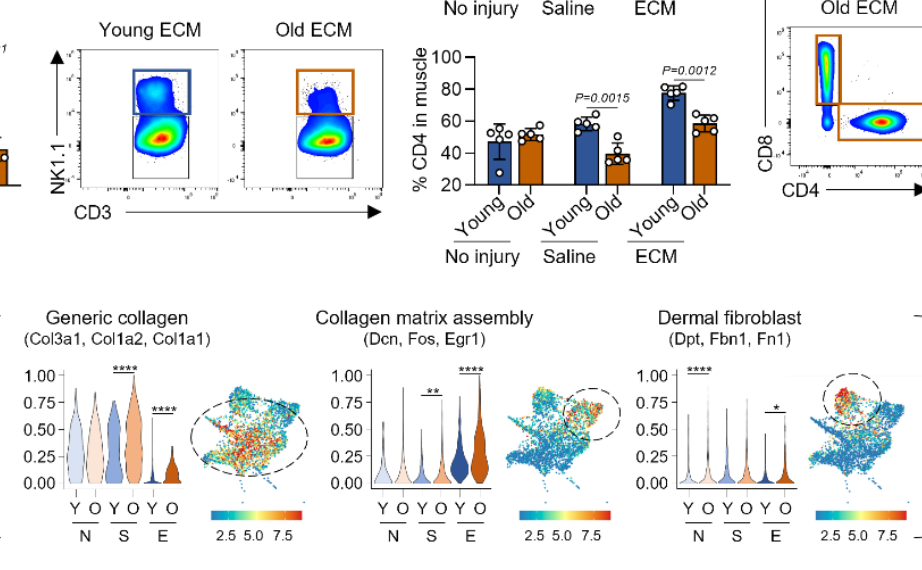

Dermal fibroblas

Non-negative matrix
factorization (NMF)
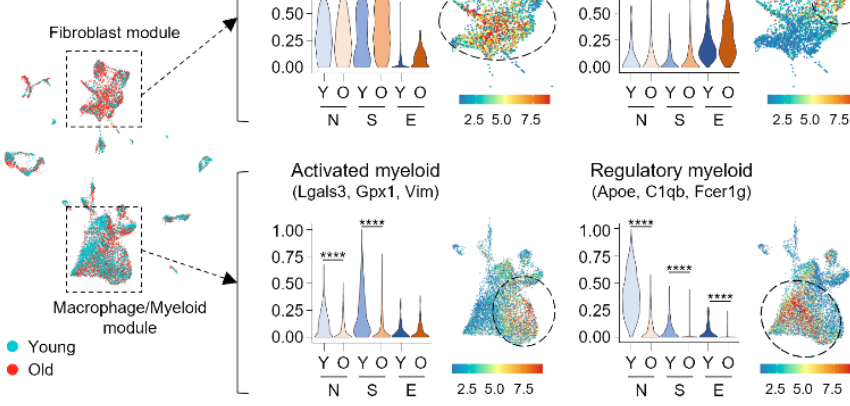

Antigen-presentation
(Cd74, H2-Aa, H2-Eb1)

100

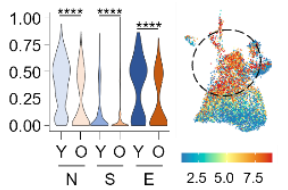

Figure 1. Aging alters immune-stromal response to the regenerative ECM biomaterials. a, Schematic illustration of experimental design including no injury control $(\mathrm{N})$, volumetric muscle loss injury treated with saline $(\mathrm{S})$ and VML treated with ECM in young (6 wk) and old (72 wk). b. Spectral flow cytometric analysis presented as UMAP plots of total live cells in muscle 1 week after ECM treatment showing immune and stromal cell populations as a percentage of total cells $(n=5)$. The granulocyte population includes eosinophils, neutrophils, mast cells, and basophils. T cells include CD4, CD8, $\gamma \delta$, and NKT cells. B cells include CD19 ${ }^{+} \mathrm{B} 220^{+}$, CD19 $\mathrm{B}^{2} 20^{+}, \mathrm{CD} 19^{+} \mathrm{B} 220^{-}$cells. $\mathbf{c}$, Quantification of immune cells from spectral flow cytometry with significant population changes between

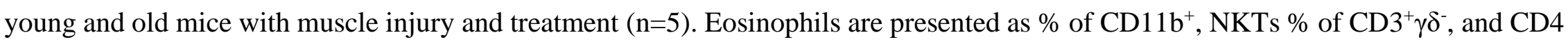


or $\mathrm{CD} 8 \%$ of $\mathrm{CD}^{+} \gamma \delta^{-} \mathrm{NK}^{-}$. d, UMAP overview (left) and classification of cell clusters with key gene signatures (right) identified using composite scRNA-seq data set on muscle from young and aged animals with injury and treatment (3 mice pooled for each condition). e, NMF-CoGAPS analysis of fibroblast populations (top) and myeloid/macrophage populations (bottom). Region of cells expressing high levels of the gene sets are circled. Flow cytometry was evaluated with two-way ANOVA with Tukey's multiple comparisons test in (c). $p$ values in NMF-CoGAPS were determined using Mann-Whitney $U$ test and adjusted with false discovery rate correction for multiple testing in (e). $*_{p}<0.05, * * p<0.01$, and $* * * * p<0.0001$. For all bar graphs, data are mean \pm s.d. 
a.

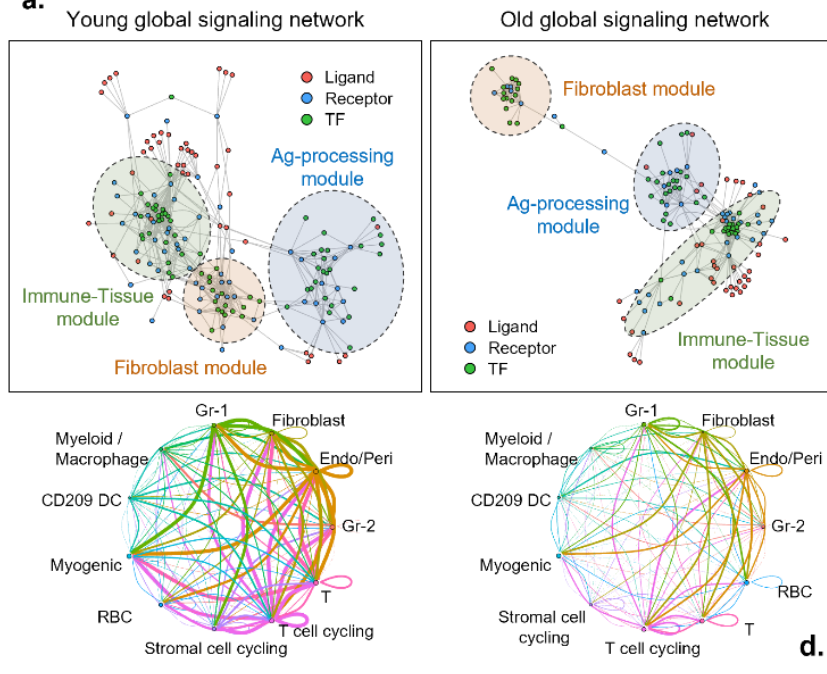

c.

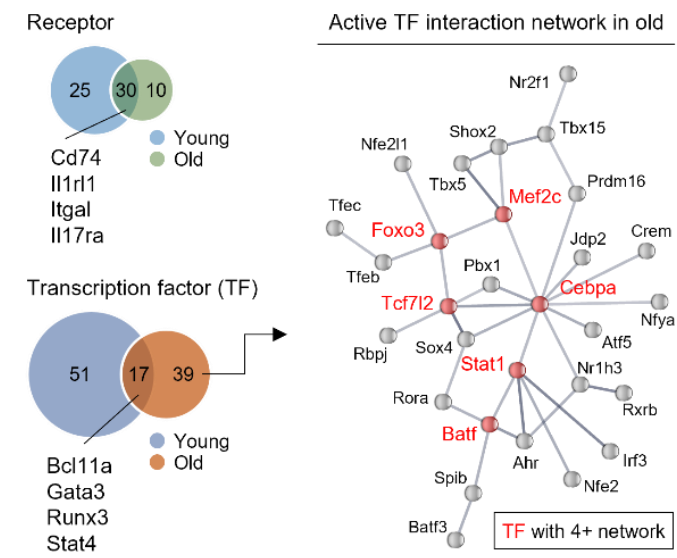

b.

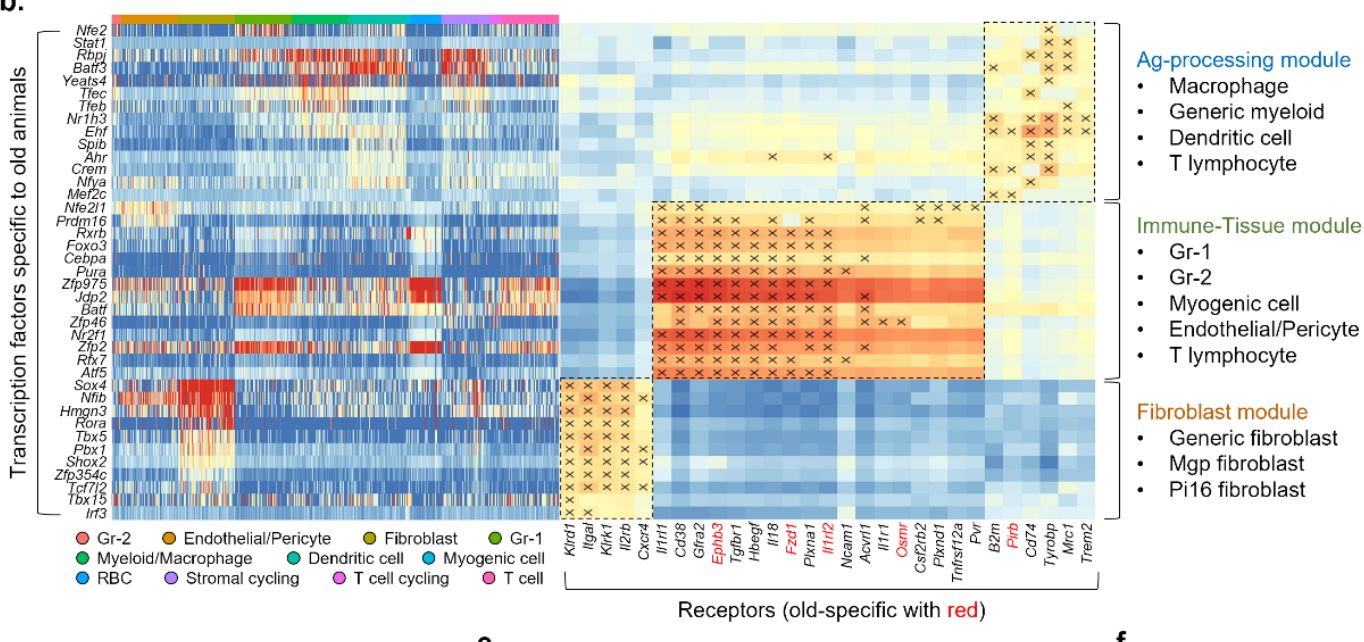

Gene set enrichment on young-specific TF
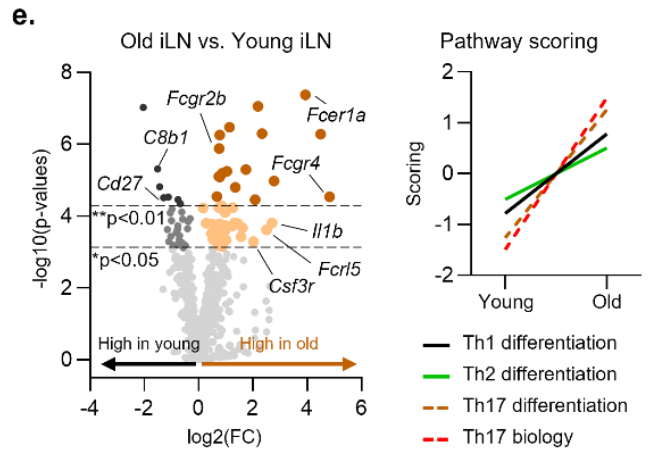

40 IFNY IL17A

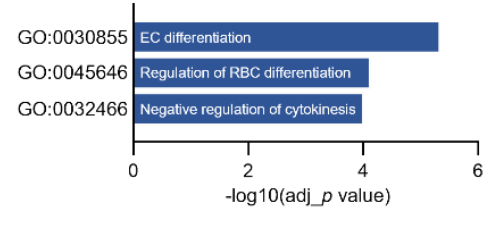

Gene set enrichment on old-specific TF

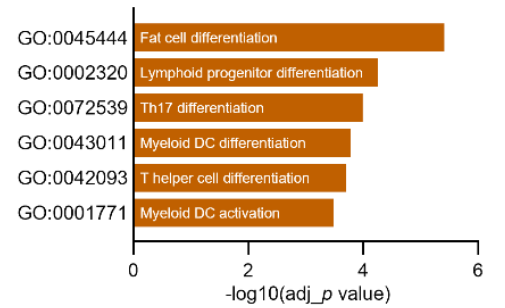

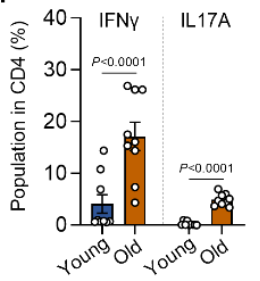

- Th1 differentiation

- Th2 differentiation

-- Th17 differentiation

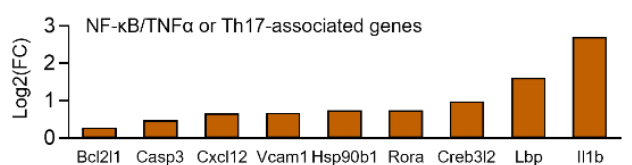

Figure 2. Aging induces a Th17-associated transcription network and impairs immune-stromal communication in muscle. a, Age-specific global signaling network (top) and inter-cluster correlation determined by Domino from the scRNA-seq demonstrating the variety and strength of the communication interactions (bottom). b. Heatmaps of activation score for age-specific TFs and its correlation 
with receptor expression. TFs specific to aged animals are labelled on the left, and the correlated receptors are labeled on the bottom right in black (receptors shared between young and aged animals) or in red (receptors specific to aged animals). Three modules of cell populations are defined based on the enrichment of receptors and TFs. c, Venn diagram illustrating shared or age-specific receptors and TFs (left), and protein-protein interaction network for the TFs specific to aged animals using STRING network (right). d, Gene set enrichment analysis using Enrichr of age-specific TFs in muscle. Adjusted $p$ value (log 10) of significant GO terms are shown. e, Volcano plot of genes expressed in aged lymph node normalized to those in young lymph node (top left). Gene scoring for helper T cell pathways (top right) or differentially expressed genes for NF-kb/TNF $\alpha$ or Th17-associated pathways (bottom) based on Nanostring analysis $(n=6)$. f, Multiparametric flow cytometry quantification of IFN $\gamma^{+}$or IL17A ${ }^{+}$CD4 or $\gamma \delta$ T cells in the lymph nodes from young or aged animals without the treatments $(n=9$ CD4 T; $n=6 \gamma \delta$ T). Unpaired two-tailed t-test (f). For all bar graphs, data are mean \pm s.e.m. 
a.

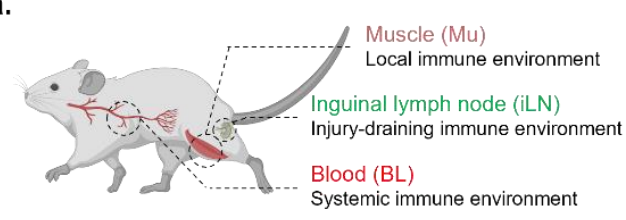

b.

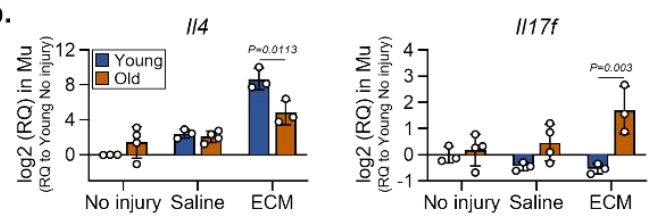

e.

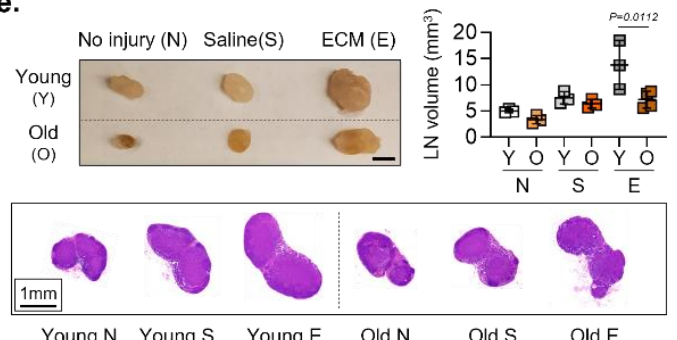

f.
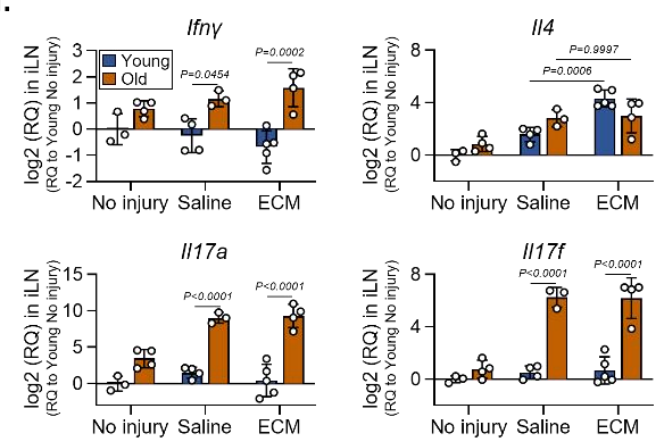

c.

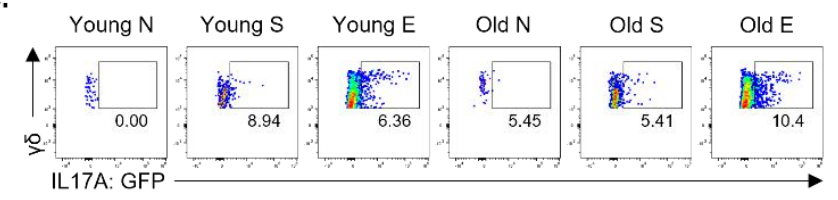
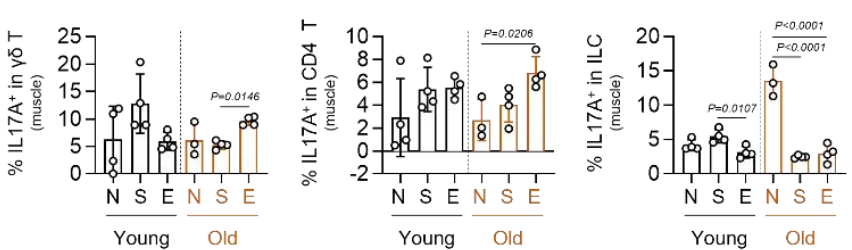

g.
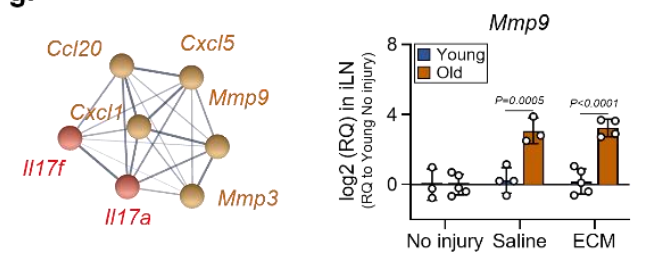

h.

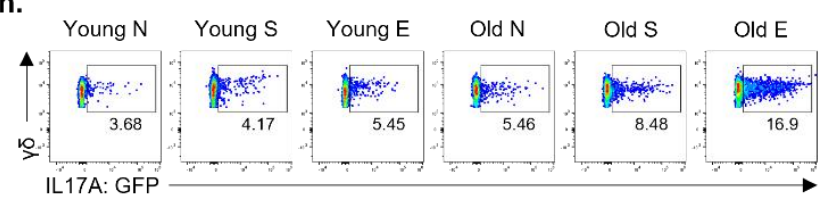

Mmp3
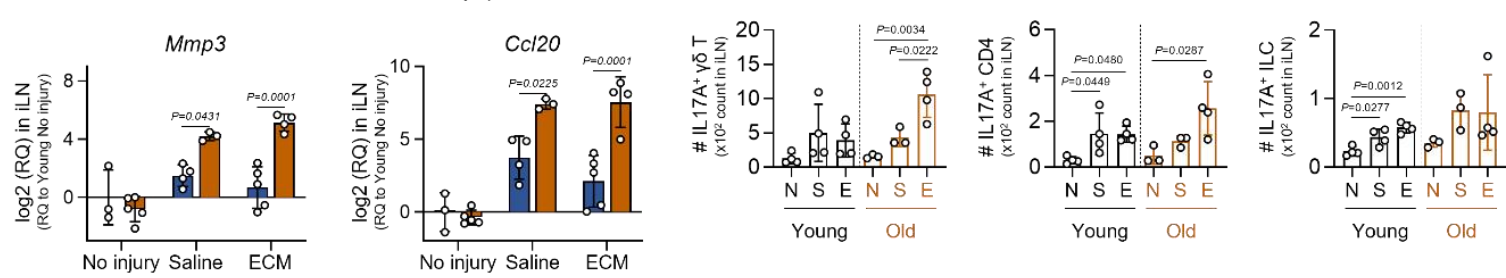

i.

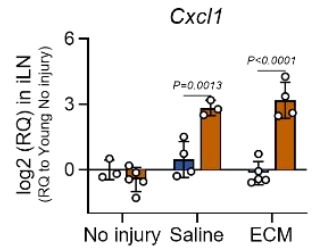

d.

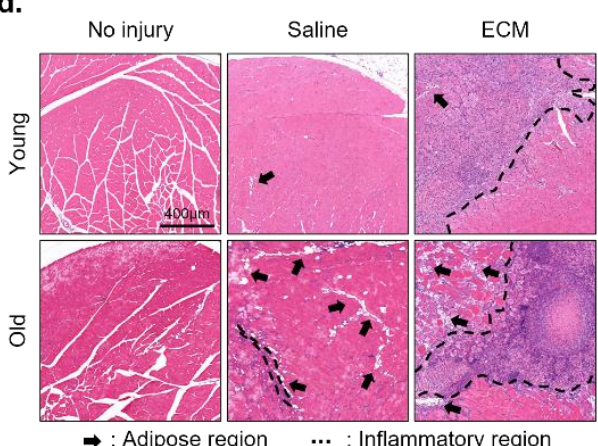

Cxc/5

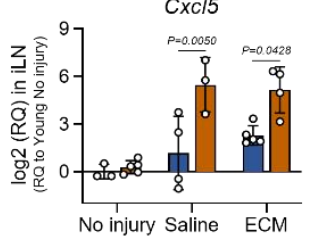

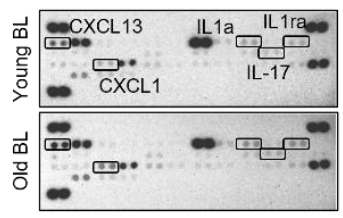

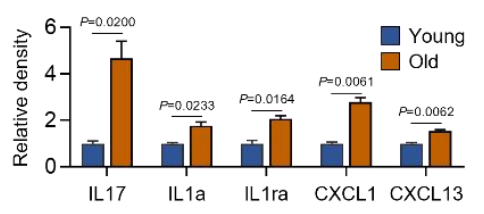

Figure 3. Injury and ECM treatment in aged animals promotes local and systemic IL17-associated immune response that inhibits

tissue repair. a, Schematic illustration of various immune environments that are affected by injury. b-d, in muscle 1 week after injury or ECM treatment. b, Quantification of Th2 or Th17-related genes in muscle ( $n=3$ young; $n=3-4$ old). $\mathbf{c}$, Representative images of flow 
cytometry comparing IL17 $\mathrm{A}^{+} \gamma \delta \mathrm{T}$ cells between young and aged animals (top) and the quantification of IL17 producing cell subtypes in muscle ( $\mathrm{n}=3-4) ; \gamma \delta \mathrm{T}$ cell, CD4 T cell, and innate lymphoid cell (ILC). d, Transverse section of the quadricep muscle stained with H\&E. The black arrow indicates the ectopic adipogenesis region, and the dotted line demonstrates immune cell infiltrated area. e-h, in lymph node 1 week after injury or ECM treatment. e, Representative images of the lymph nodes 1 week after the treatments (top left) and the quantification of their size (top right). Scale bar, $2 \mathrm{~mm}$. Transverse section of the lymph nodes is stained with H\&E (bottom). $\mathbf{f}$, Quantification of T helper cell cytokine-related genes in lymph node ( $\mathrm{n}=3-5$ young; $\mathrm{n}=3-4$ old). g, Quantification of Th17-associated genes in the lymph nodes $(n=3-5)$. Protein-protein interaction demonstrating their association with IL17A or IL17F are shown using STRING network (top left). $\mathbf{h}$, Representative images of flow cytometry data comparing IL17A ${ }^{+} \gamma \delta$ T cells between young and aged animals (top) and the quantification of IL17 producing cell subtypes in the lymph node (n=3-4). i, Representative images (left) and quantitative analysis (right) of the protein array performed on blood serum from young and aged mice without the treatments ( 3 mice pooled for each condition). Protein molecules with significant differences in pixel densities compared to young animals are labeled and quantified using imageJ. Two-way ANOVA with Tukey's multiple comparisons test (b, e, f, g), one-way ANOVA with Tukey's multiple comparisons test $(\mathbf{c}, \mathbf{h})$, unpaired two-tailed t-test (i). For all bar graphs, data are mean \pm s.d. 
a.

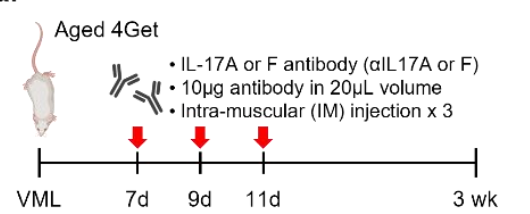

c.
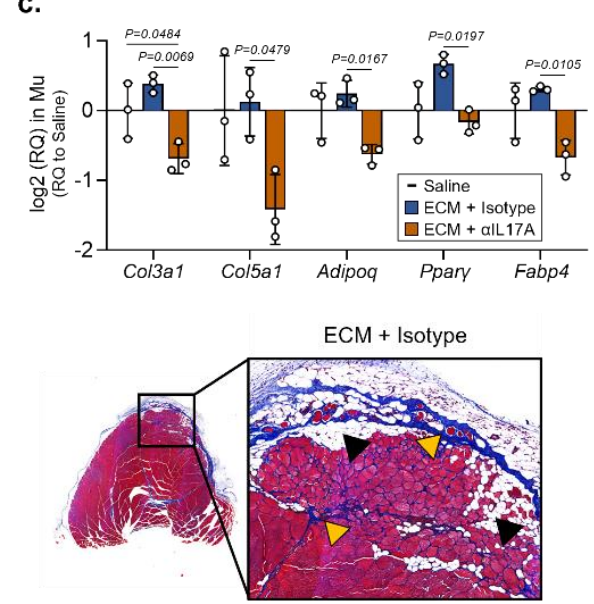

b.
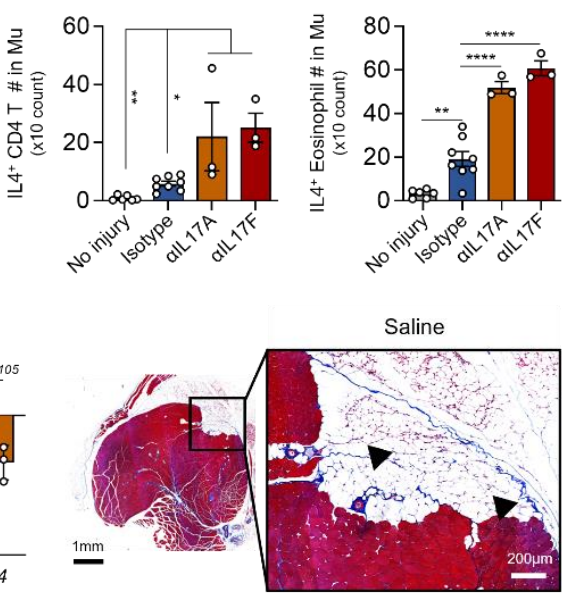

Adipose deposition

$E C M+$ alL17A

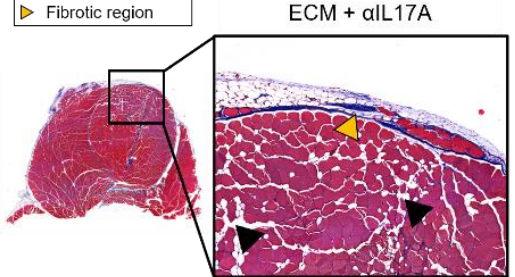

Aged Aged
C57BL6J 4Get

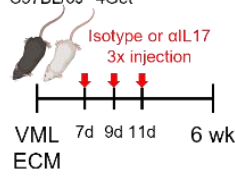

d.

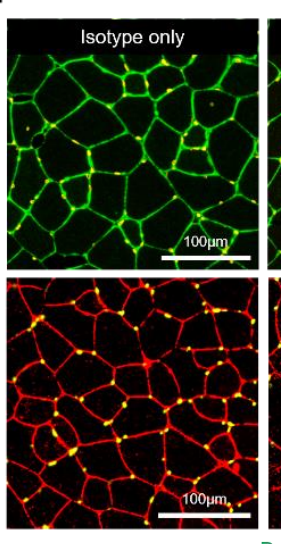

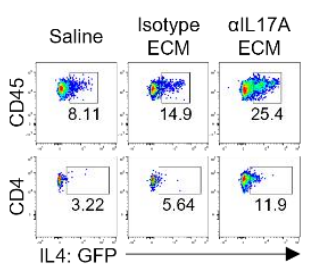
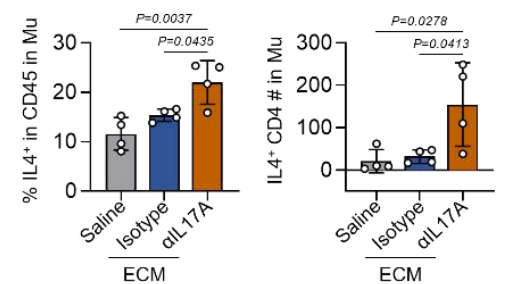

$\mathrm{ECM}$

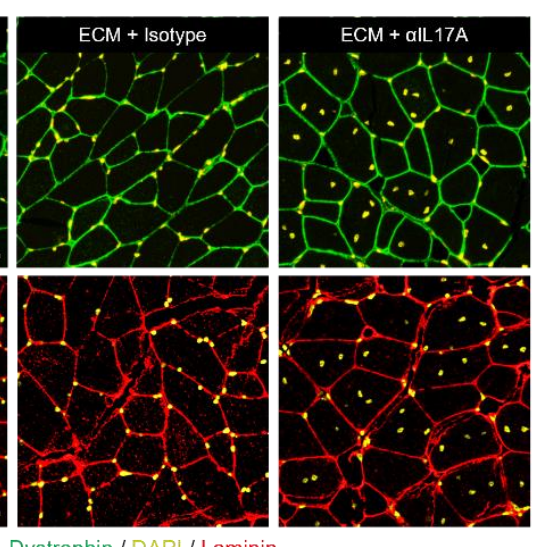

Figure 4. Local IL17 suppression rejuvenates the type 2 immune response to injury and ECM to restore tissue repair and reduce

fibrosis in old animals. a, Schematic illustration of experimental design (left) and quantification of flow cytometry data for IL4 ${ }^{+}$CD4 $\mathrm{T}$ cells and eosinophils in muscle 3 weeks after injury (right; $\mathrm{n}=6$ no injury; $\mathrm{n}=8$ isotype; $\mathrm{n}=3 \alpha \mathrm{IL} 17 \mathrm{~A}$ and $\alpha$ IL17F). b, Experimental schematics (left), and representative images flow cytometry showing IL4 $4^{+} \mathrm{CD} 45$ or CD4 T cells (middle) and quantification of IL4 ${ }^{+}$cell populations in muscle 6 weeks after injury and ECM treatment (right; $n=4$ ). $\mathbf{c}$, Quantification of genes associated with fibrosis or adipogenesis in muscle (top left; $n=3$ ), and transverse section of the quadricep muscle 6 weeks after injury stained with Masson's Trichrome. d, Immunofluorescence images of the quadricep muscle 6 weeks after injury stained with dystrophin (top) or laminin (bottom). Quantification of muscle fibers with central nuclei are shown (right; $\mathrm{n}=3$ ). Nuclei were stained with DAPI (represented in 
yellow). One-way ANOVA with Tukey's multiple comparisons test (a-d). $* p<0.05, * * p<0.01$, and $* * * * p<0.0001$. For all bar graphs, data are mean \pm s.e.m (a) or s.d. (b-d). 


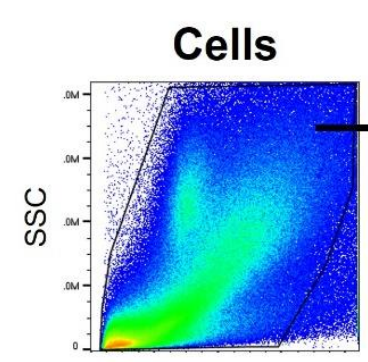

FSC
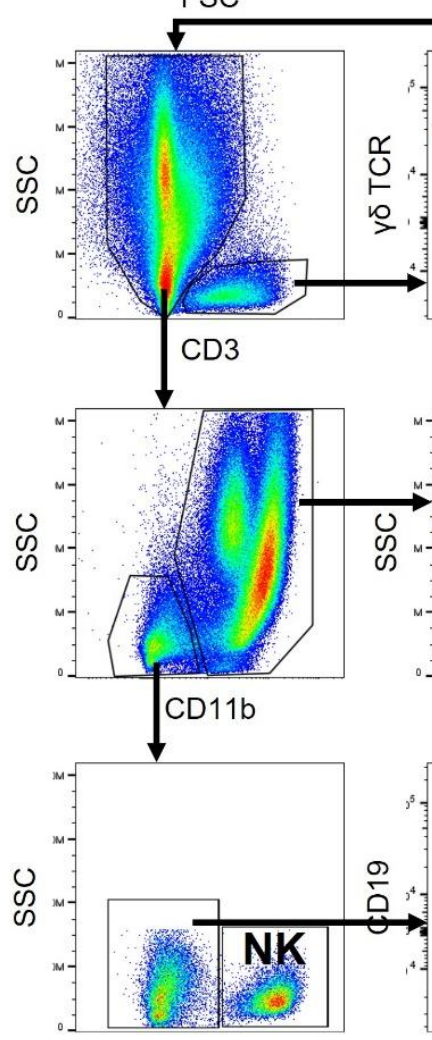

NK1.1

\section{Single cell}

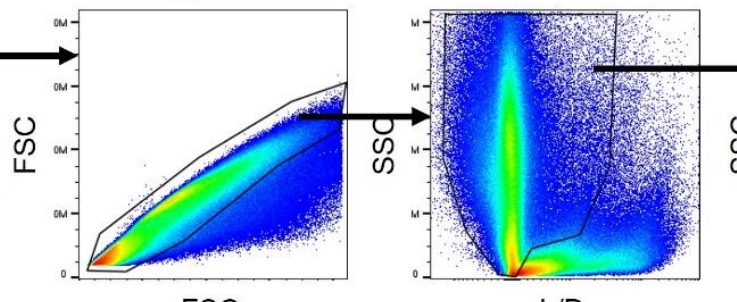

FSC

L/D

CD45

CD45+ or -

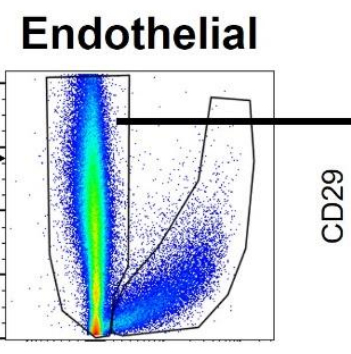

CD31

CD8

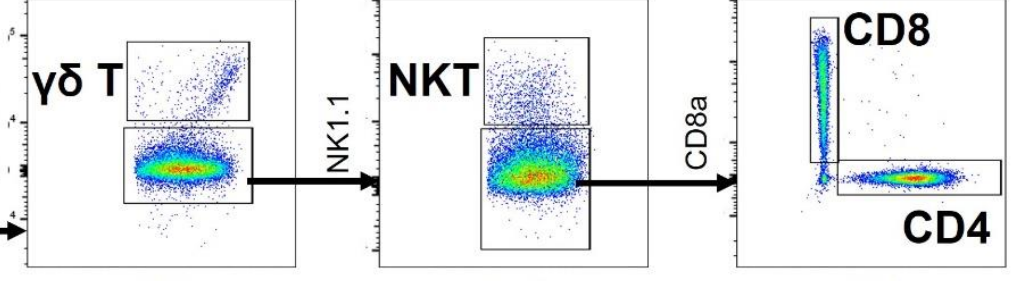

CD3

CD3

CD4

Fibroblast

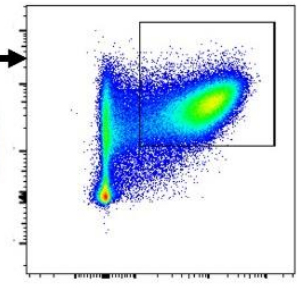

CD90. 2
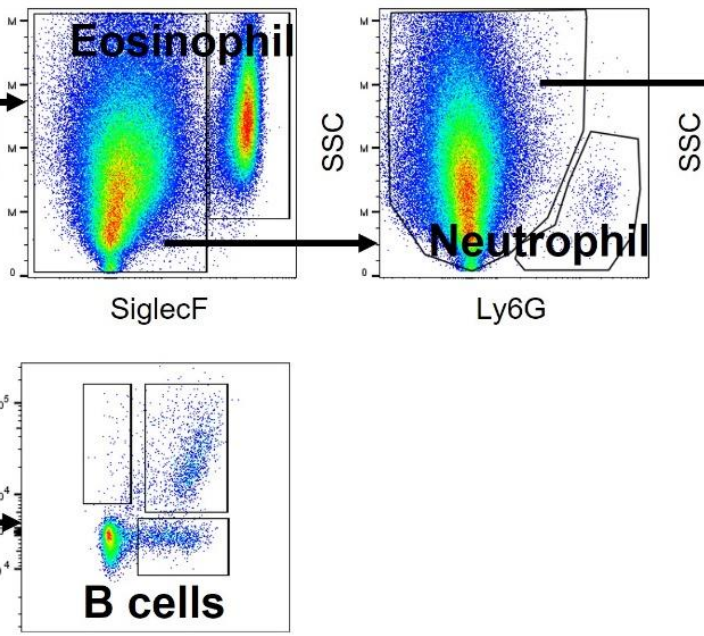

B220
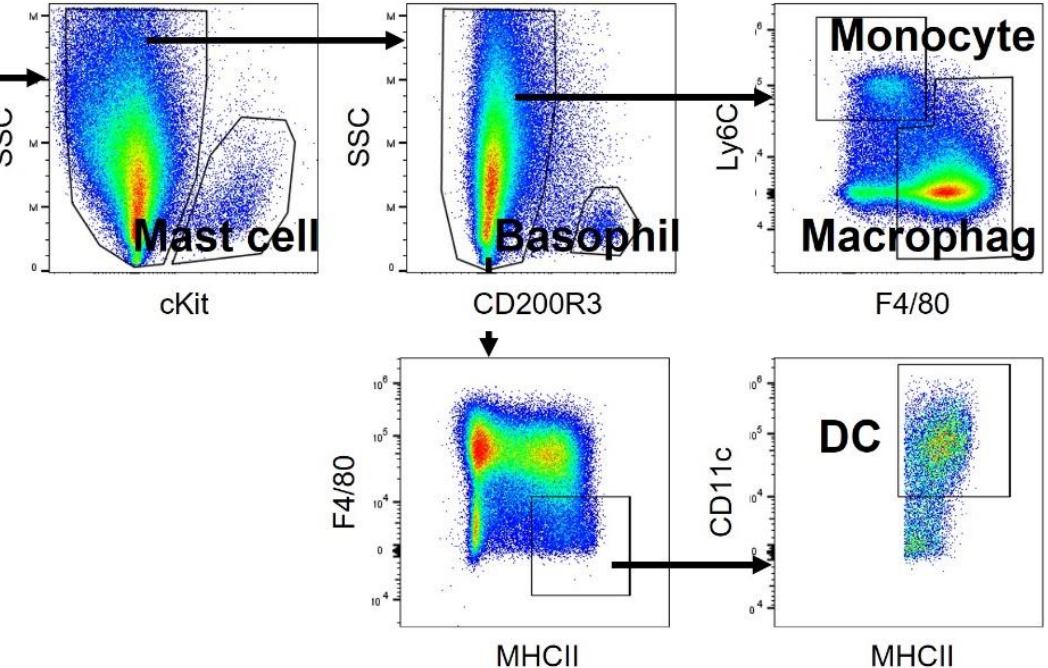

Extended data figure 1. Schematic representation of the gating strategy used to identify indicated cell phenotypes from single cell suspensions from mouse muscle tissue using spectral flow cytometry. 
a.
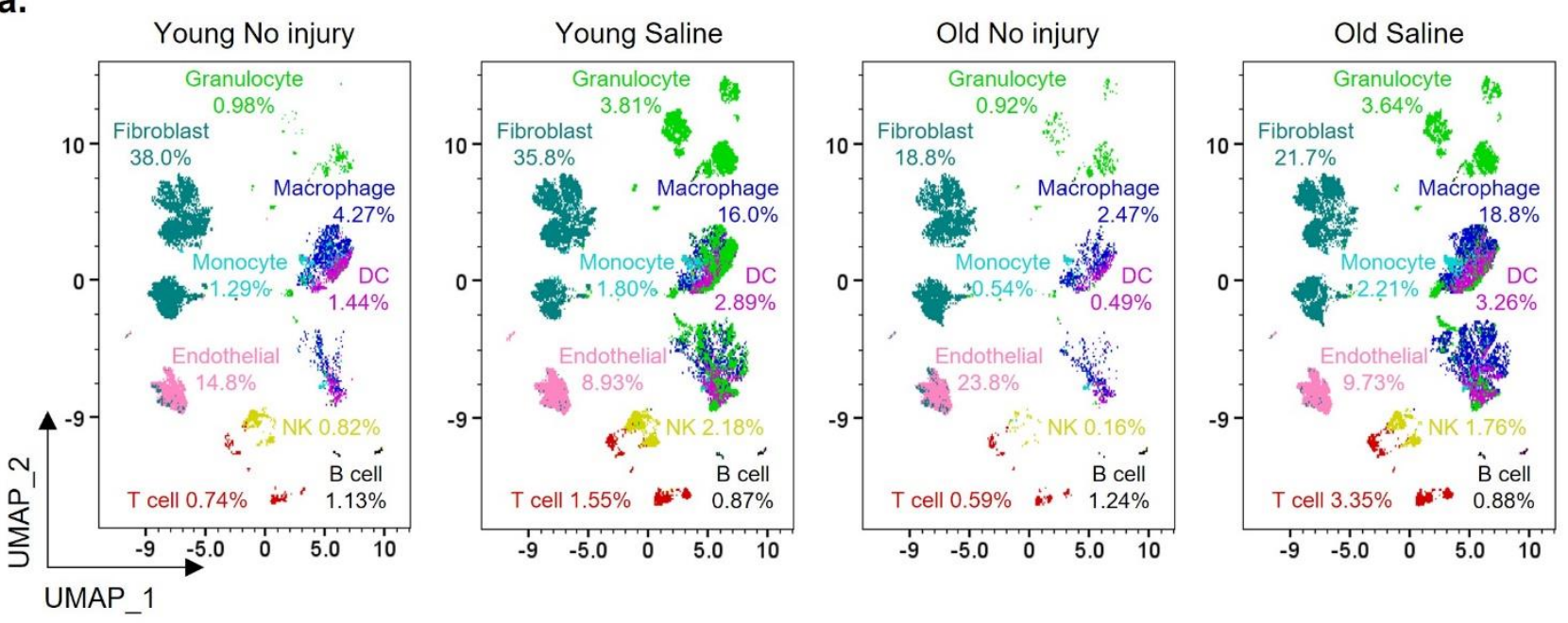

b.
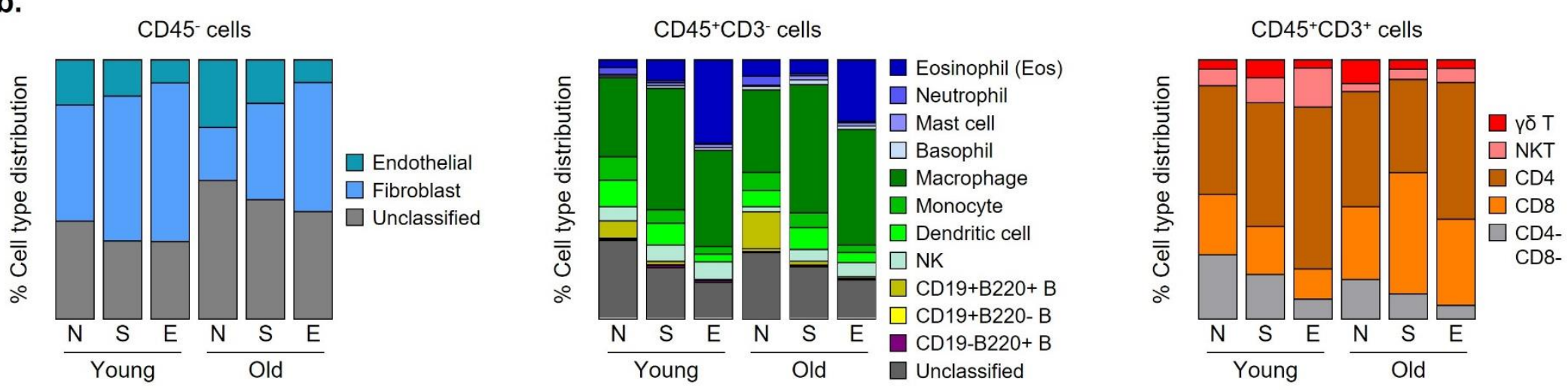

Extended data figure 2. Changes in immune and stromal cell phenotypes 1 week after injury or injury + ECM treatment between young and aged animals. a, Overview of UMAP plots from total live cells in muscle showing the population of the indicated cell phenotypes. b, Quantification of immune-stromal cells in muscle after treatment as determined by flowcytometry. (a, b) Indicated cell population represents average value of $n=5$ per group. 
CD45+ cells, macrophage/monocytes, dendritic cells, and natural killer cells

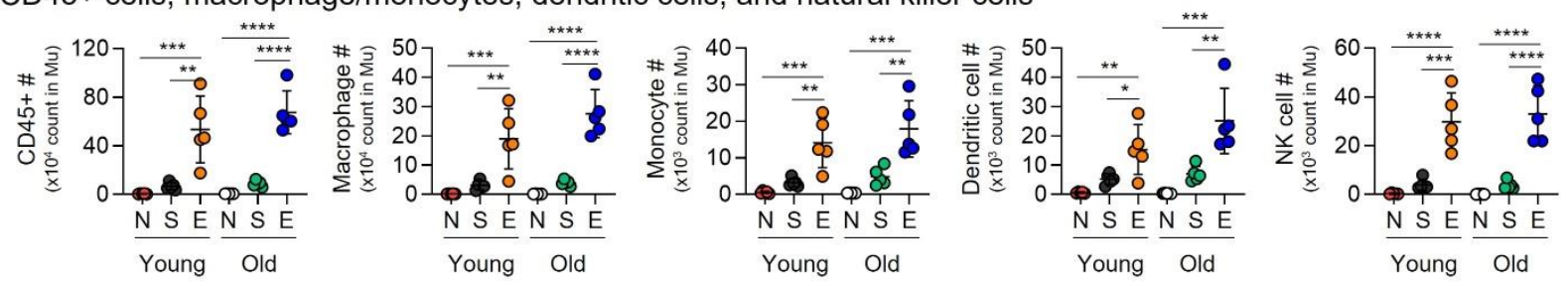

Granulocyte

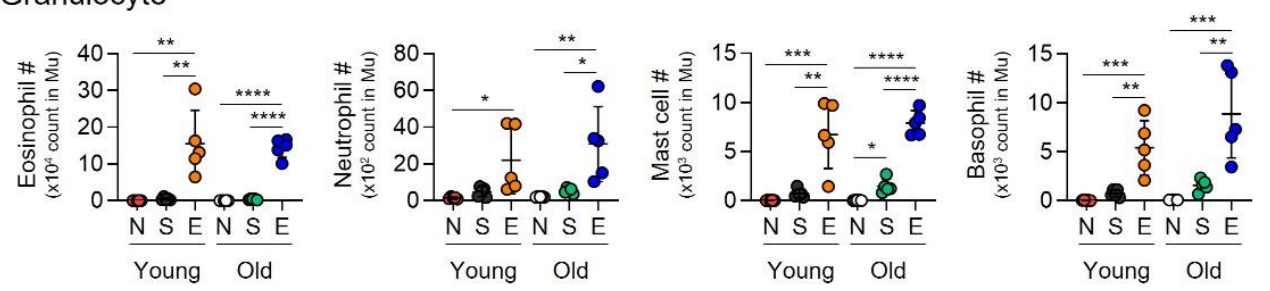

$\mathrm{T}$ and $\mathrm{B}$ cells
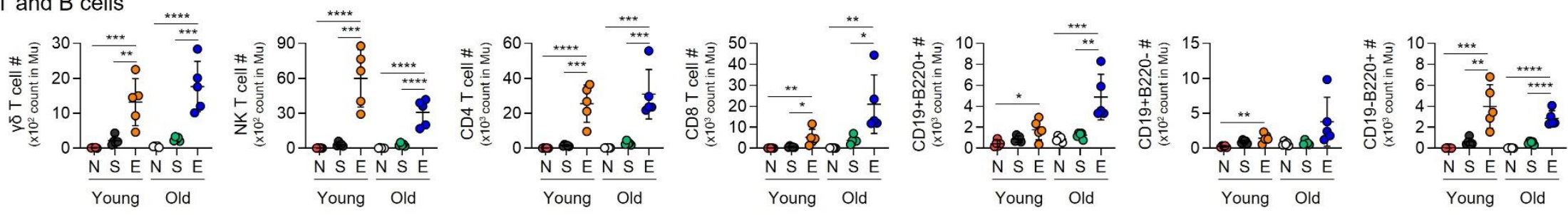

\section{CD45- cells}

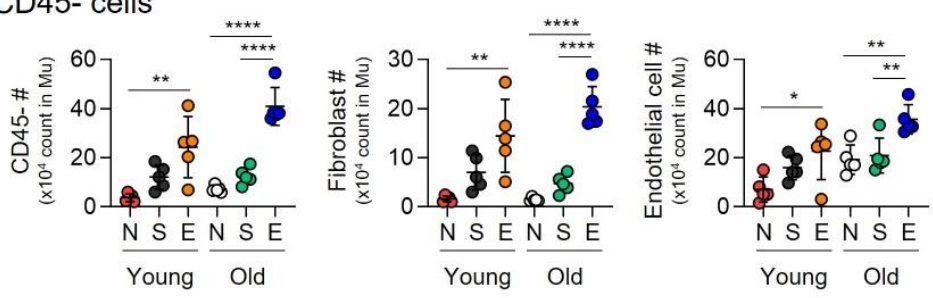

Extended data figure 3. Flow cytometry quantification (cell counts) of the indicated immune and stromal cell phenotypes in muscle from young and aged animals 1 week after injury and treatment. Statistical analysis was performed using a one-way ANOVA with Tukey's multiple comparisons test within the respective age groups $(\mathrm{n}=5)$. * $p<0.05$, ** $p<0.01$, ***p $<<0.001$, and $* * * * p<0.0001$. For all bar graphs, data are mean \pm s.d. 
a.
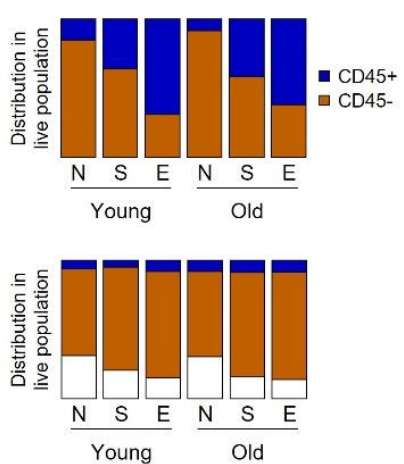

- CD45+CD3+CD11b- (lymphoid population) a CD45+CD3-CD11b+ (myeloid population) $\square$ CD45+CD3-CD11b- b.

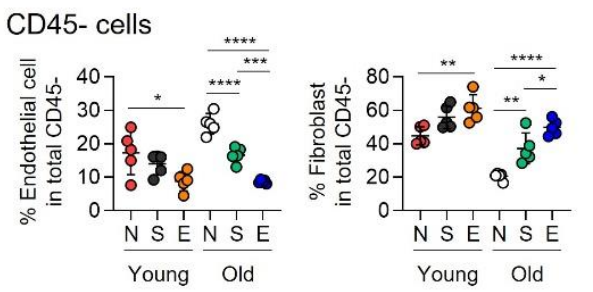

Macrophage/monocytes, dendritic cells, and natural killer cells
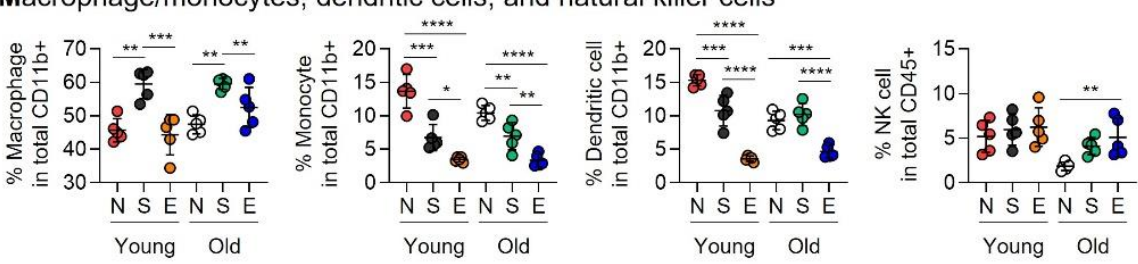

Granulocyte
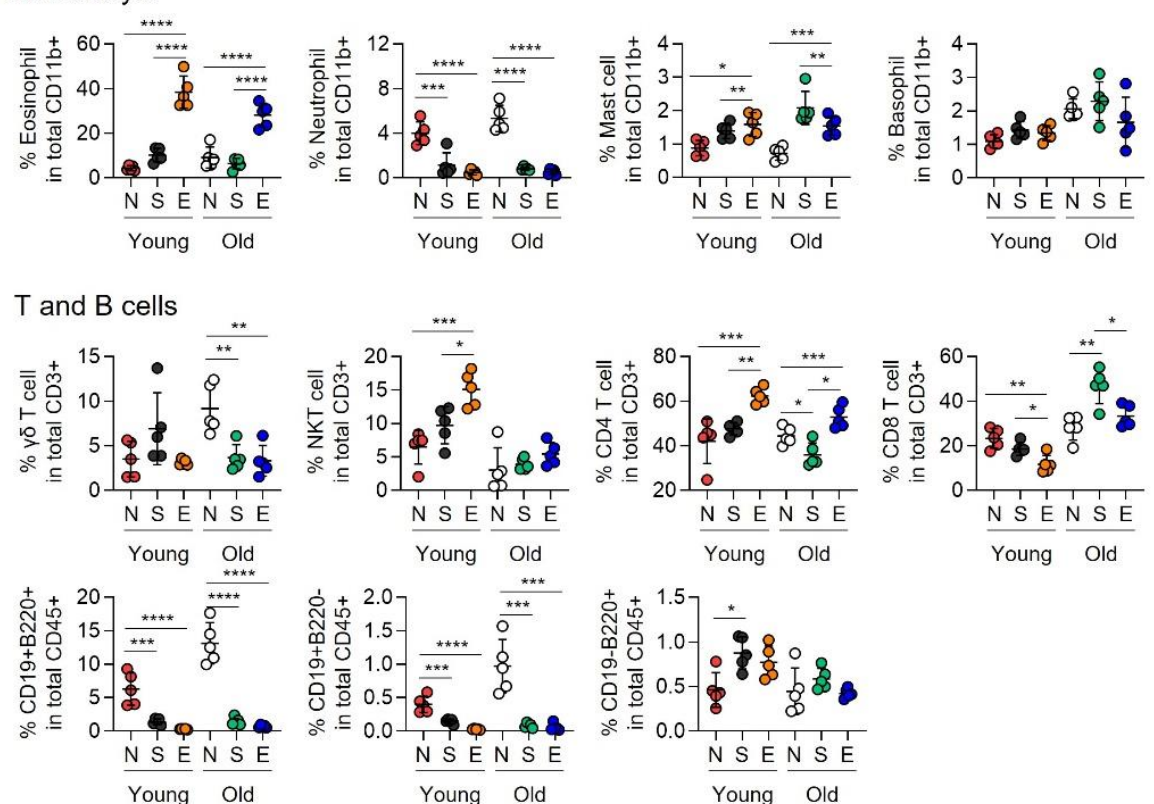

Extended data figure 4. Flow cytometry quantification (cell percentages) of a, CD45+/CD45- cells or myeloid/lymphoid cells (indicated cell population represents average value of $n=5$ per group), and $\mathbf{b}$, indicated immune/stromal cell phenotypes in muscle between young and aged animals 1 week after the treatments. Statistical analysis was performed using a one-way ANOVA with Tukey's multiple comparisons test within the respective age groups $(\mathrm{n}=5)$. ${ }^{*} p<0.05,{ }^{* *} p<0.01,{ }^{* * *} p<0.001$, and ${ }^{* * * *} p<0.0001$. For all bar graphs, data are mean \pm s.d. 
a.

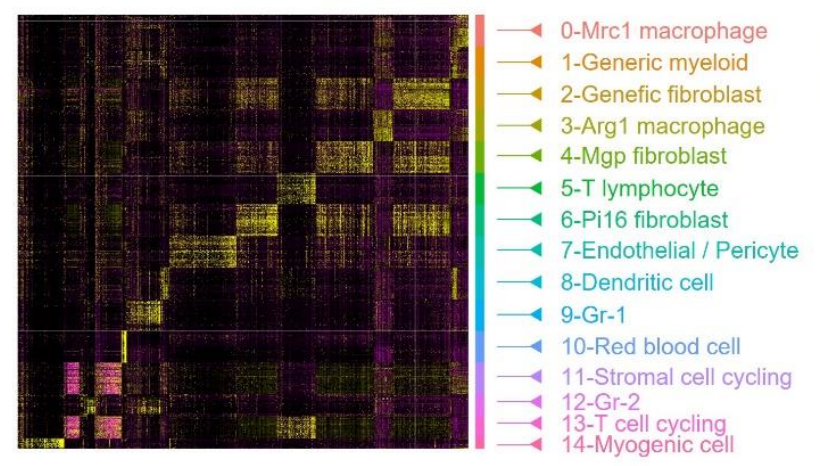

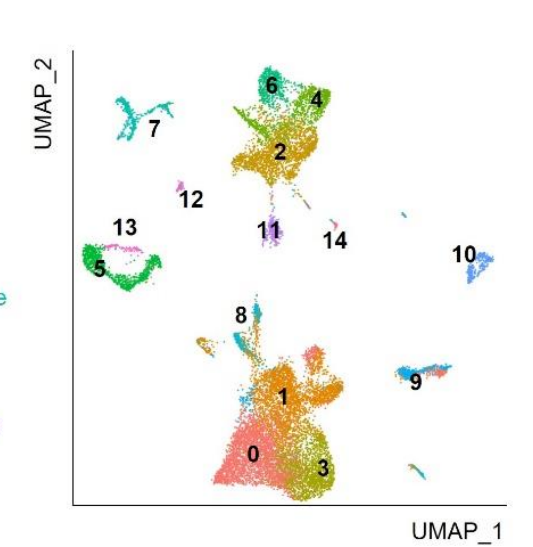

UMAP_1

b.

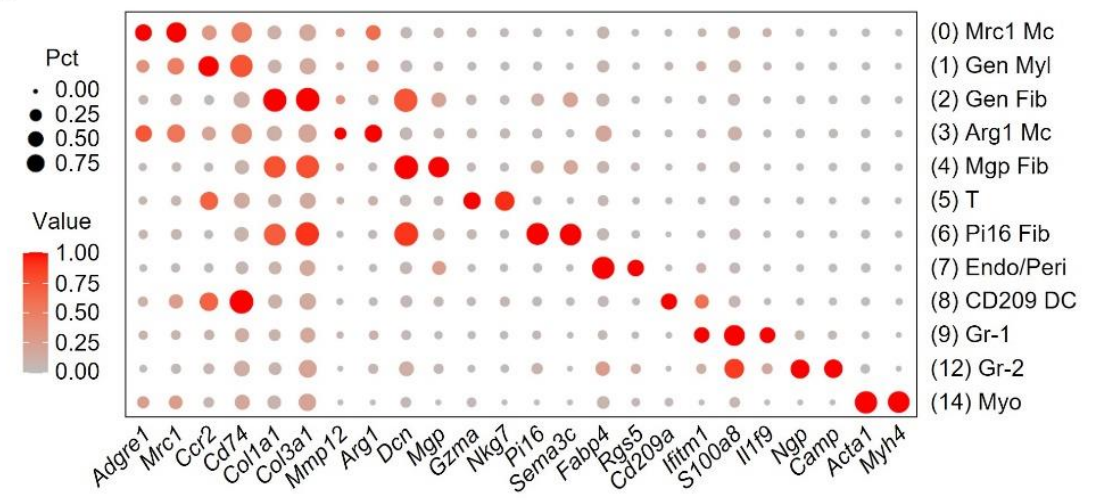

c.
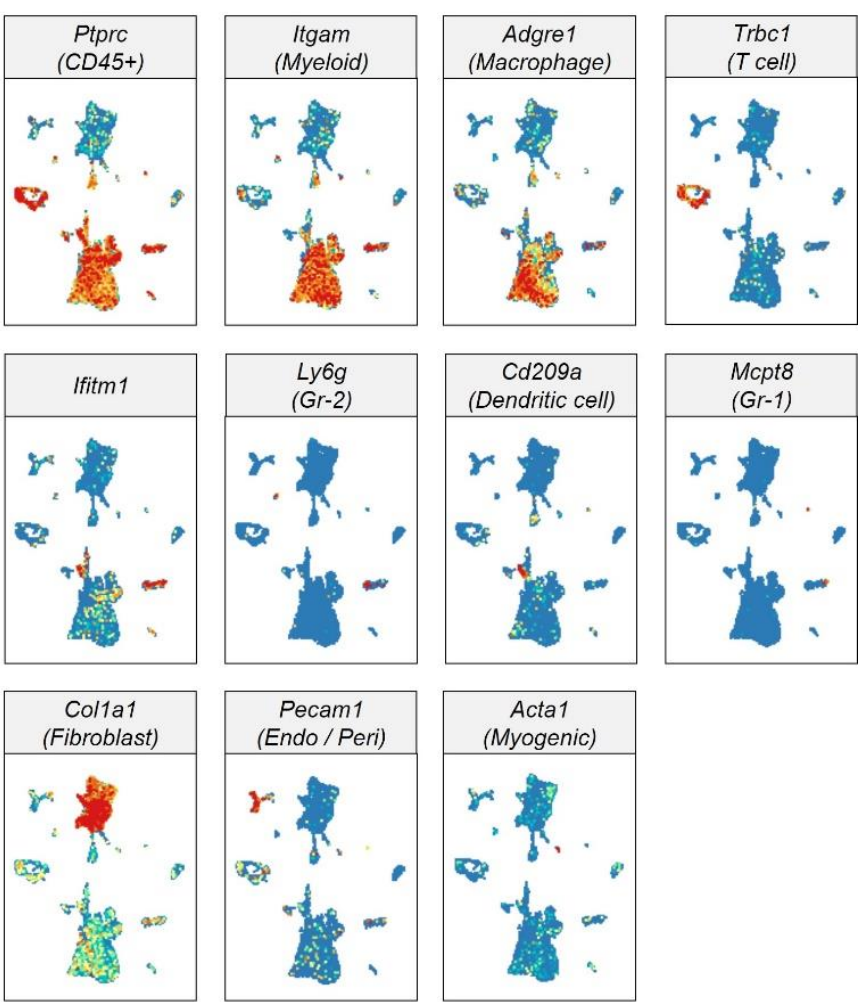

Extended data figure 5. Single cell RNA sequencing-based cell clustering information in muscle tissue 1 week after the treatments. a, Heatmap of differentially expressed genes with highest log fold-change from each cluster (left) and UMAP plot with cluster labels (right). b, Signature gene markers for single cell clusters. Each dot shows the expression of genes associated with cluster identity. Gene expression after normalization to the maximum averaged expression are shown. c, Overview of cell clusters identified in UMAP plots based on the key gene expression. 


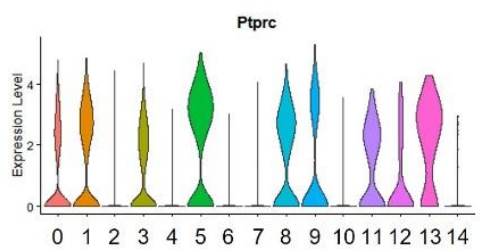

Identity
Arg1

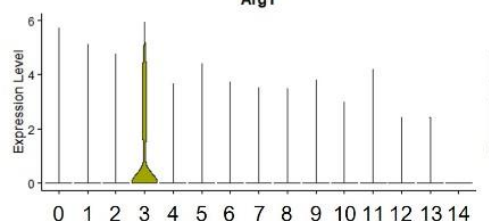

Identity

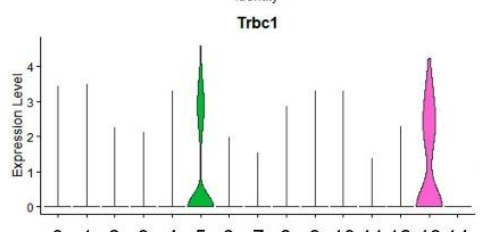

$\begin{array}{lllllllllll}0 & 1 & 2 & 3 & 4 & 5 & 6 & 7 & 8 & 9 & 1011121314\end{array}$

Identity
Thbs 4

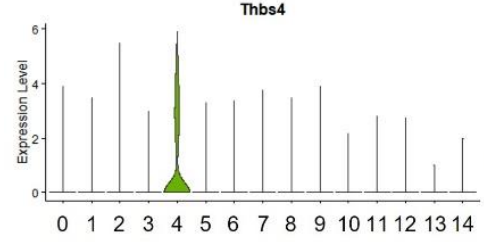

Identity

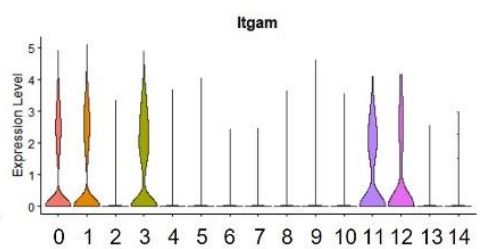

Identity

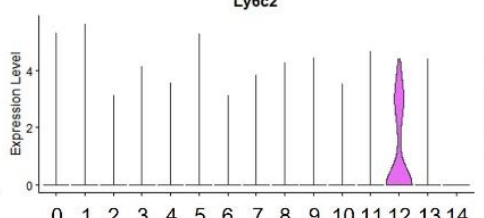

Identity

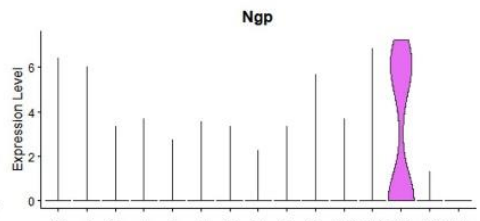

$0 \begin{array}{llllllllll}0 & 1 & 3 & 4 & 5 & 6 & 7 & 8 & 9 & 1011121314\end{array}$

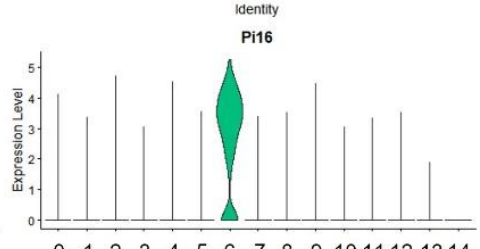

Identity

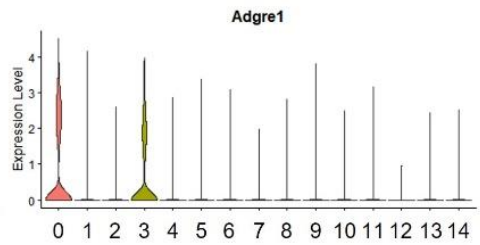

identity
II1f9

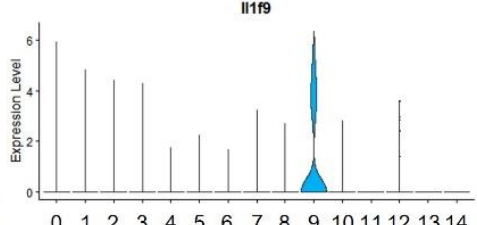

Identity
Itgb1

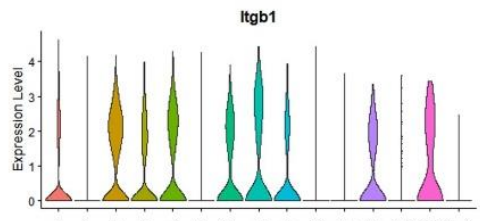

$\begin{array}{lllllllllll}0 & 1 & 2 & 3 & 4 & 5 & 6 & 7 & 8 & 9 & 1011121314\end{array}$

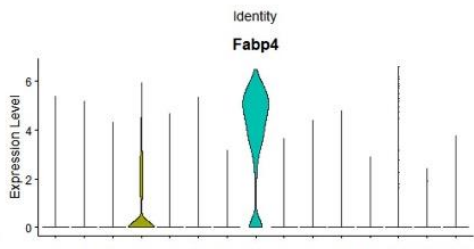

Identily

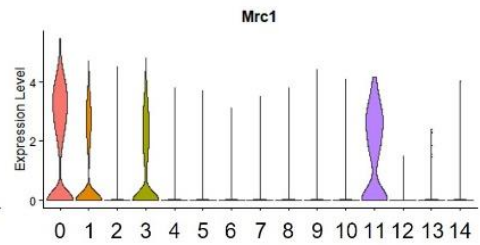

Identity

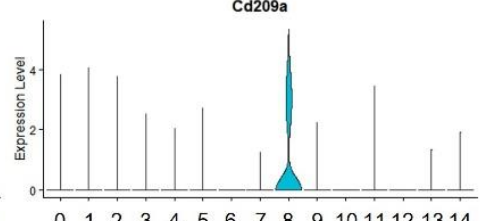

Identity

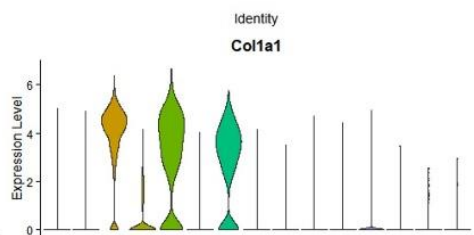

01234507801011121314

Identity

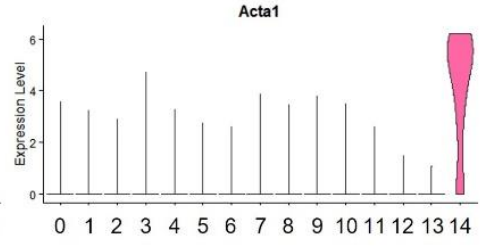

Identity
0-Mrc1 macrophage 1-Generic myeloid 2-Genefic fibroblast 3-Arg1 macrophage 4-Mgp fibroblast 5-T lymphocyte 6-Pi16 fibroblast 7-Endothelial / Pericyte -Dendritic cell 9-Granulocyte-1 -Red blood cel 1-Stromal cell cycling 12-Granulocyte-2 3-T cell cycling 14-Myogenic cell

Extended data figure 6. Gene expressions of cluster-defining markers shown in violin plots. 

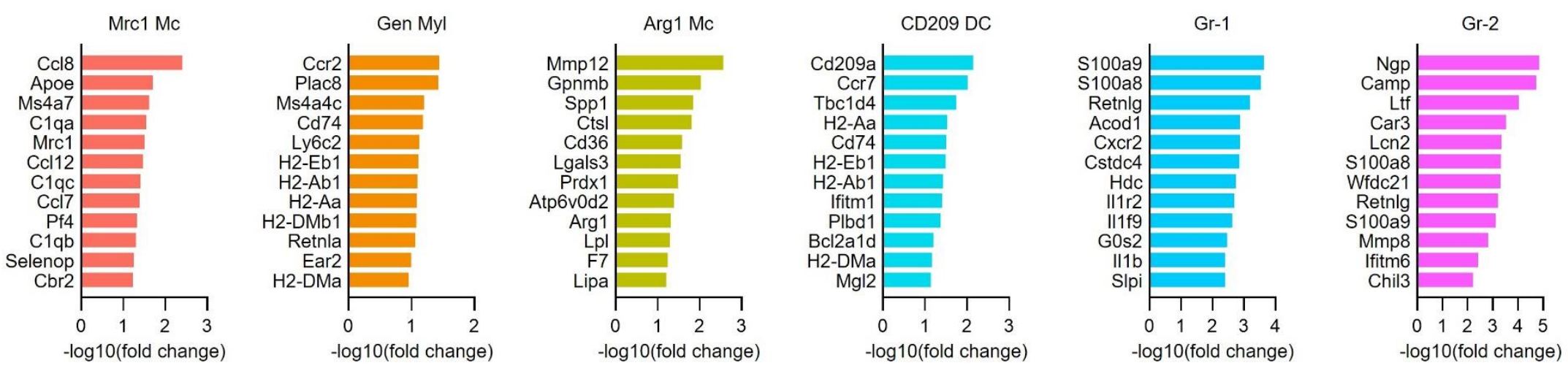

T

Gen Fib
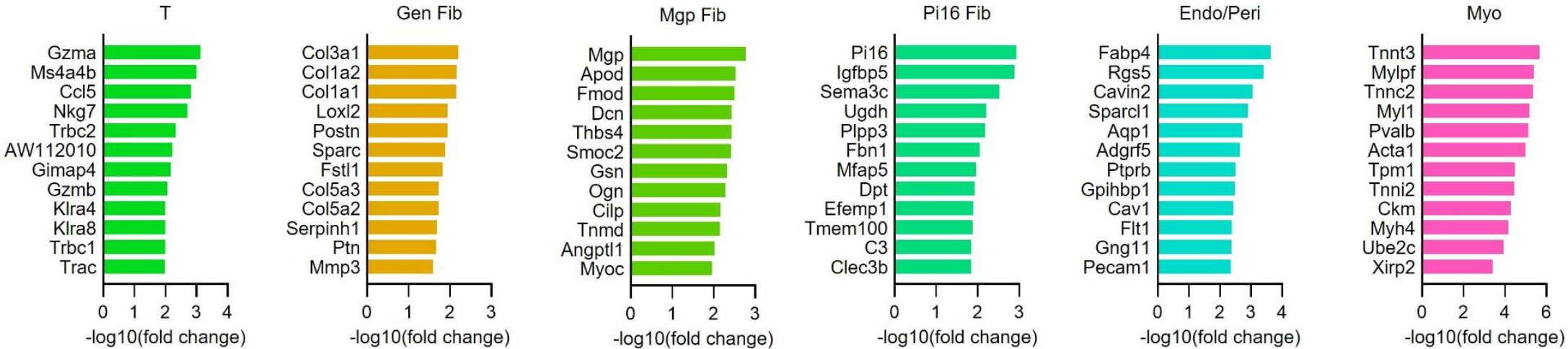

Extended data figure 7. Twelve top differentiated genes of each cluster population. Values represent $\log 10$ fold change between each cluster compared to all other clusters. 
a.
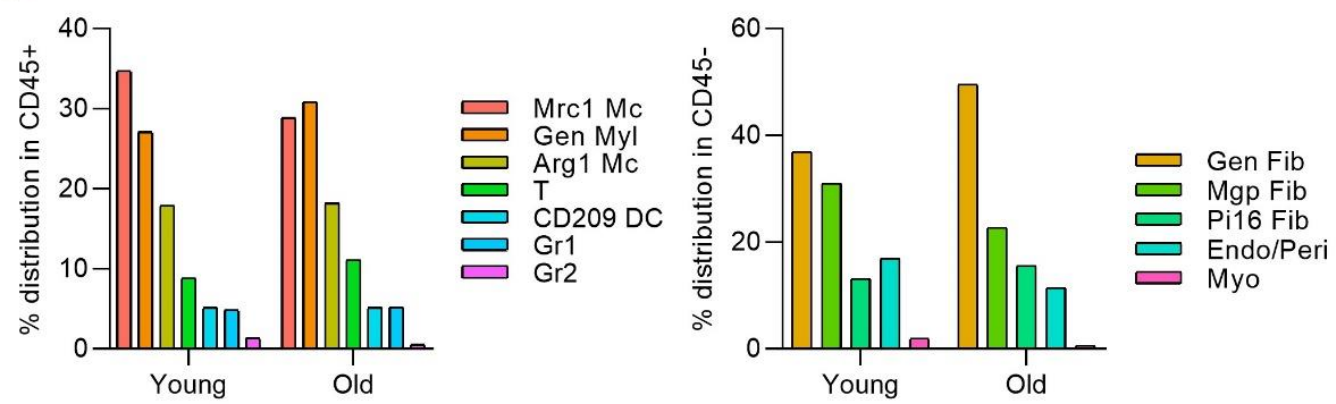

b.
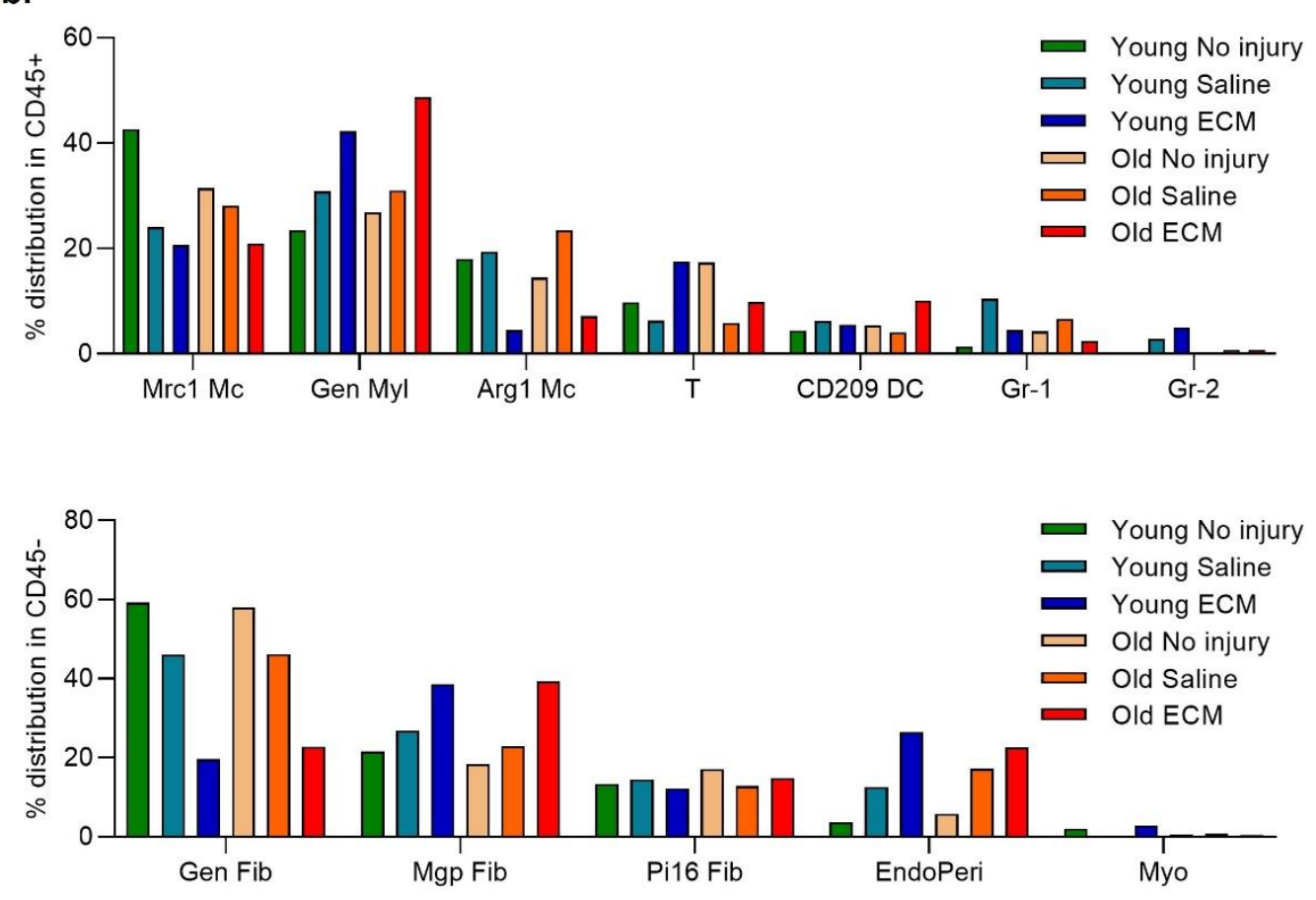

Extended data figure 8. Quantification of each cell cluster between the a, age groups or $\mathbf{b}$, treatment groups using single cell RNA sequencing dataset. 

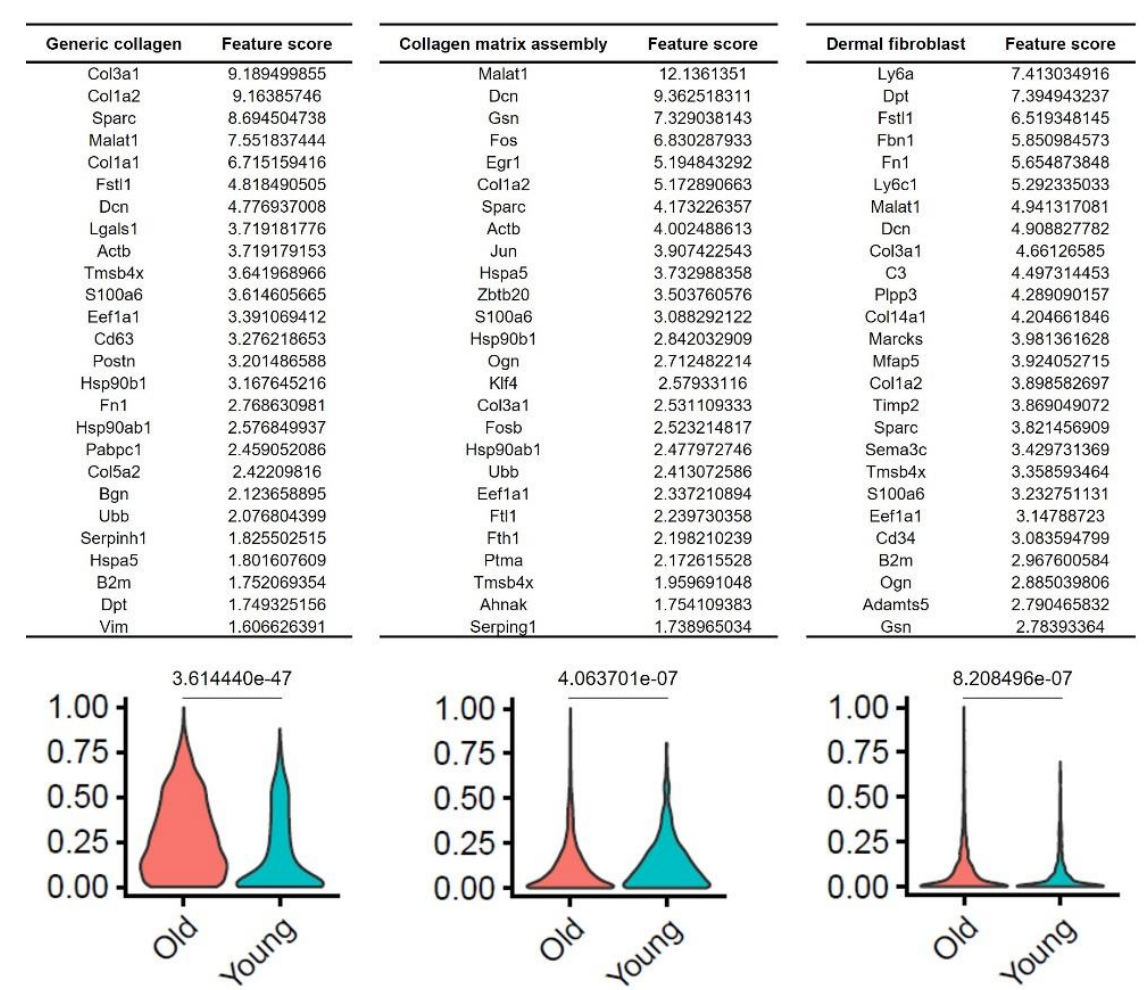
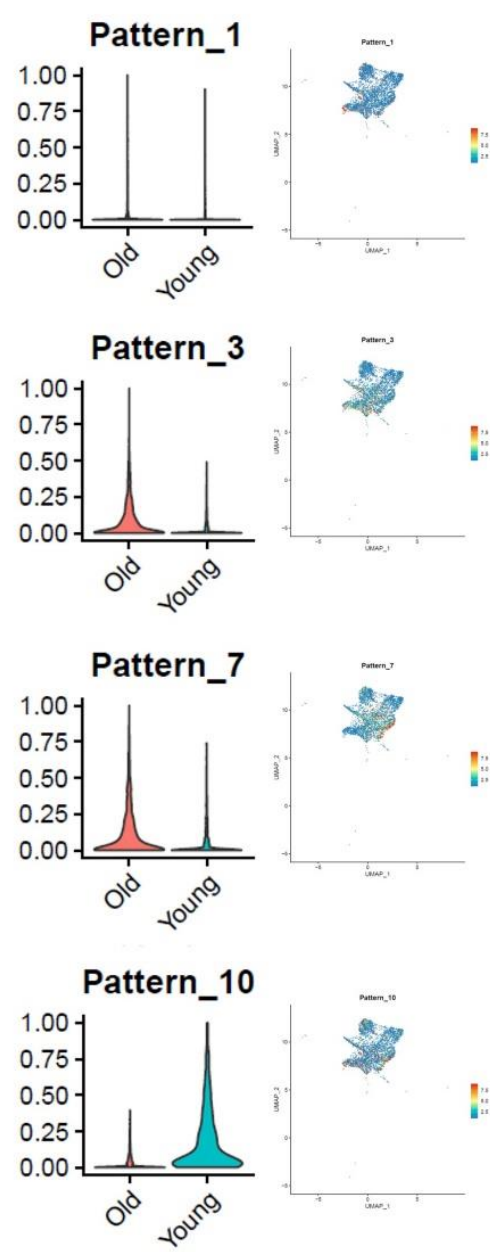
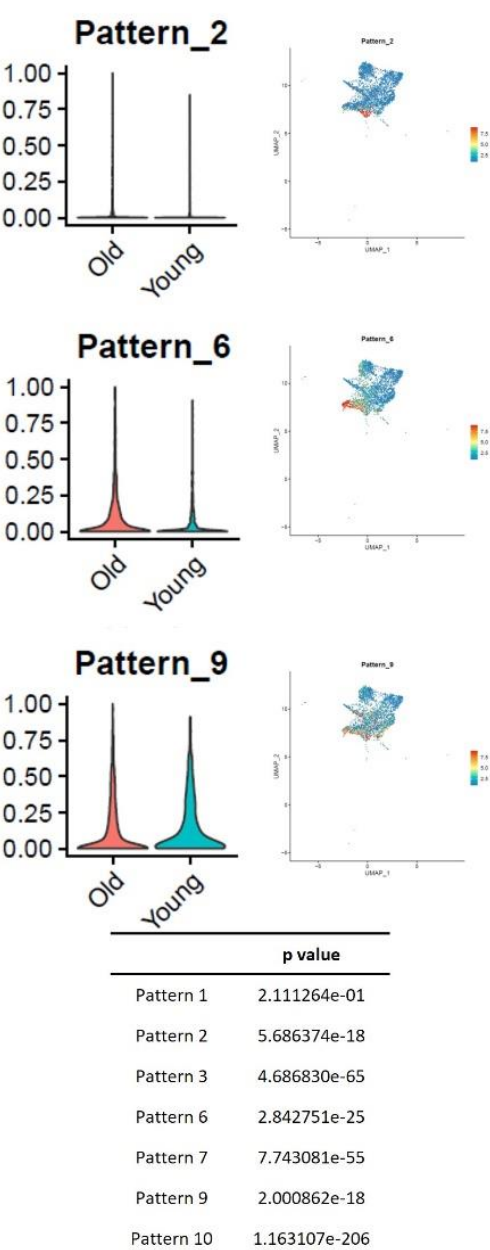

Extended data figure 9. Gene signature of fibroblast populations using NMF CoGAPS. $p$ values are determined by Mann-Whitney U test and adjusted with false discovery rate correction for multiple testing. 

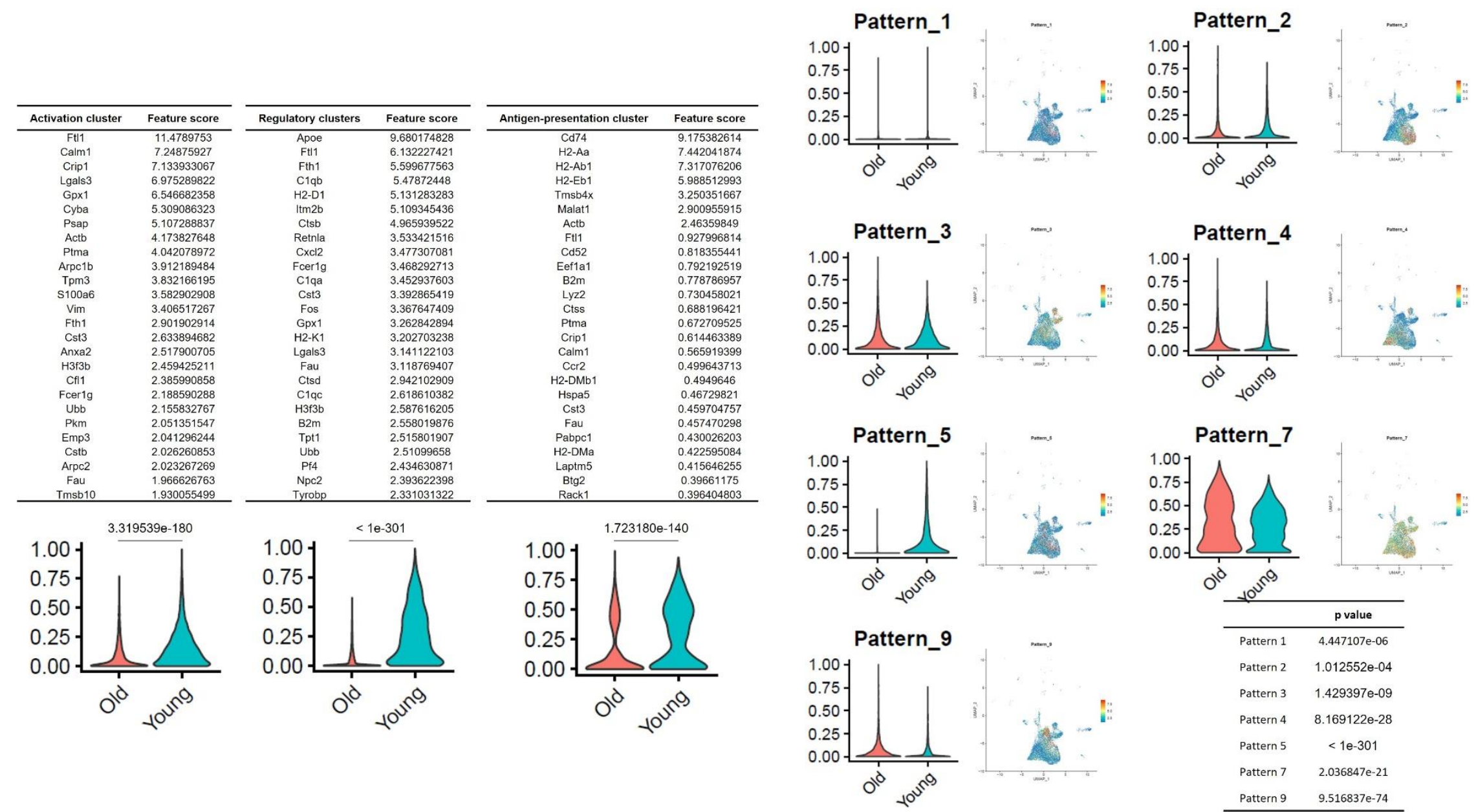

Extended data figure 10. Gene signature of myeloid/macrophage populations using NMF CoGAPS. $p$ values are determined by MannWhitney $\mathrm{U}$ test and adjusted with false discovery rate correction for multiple testing. 


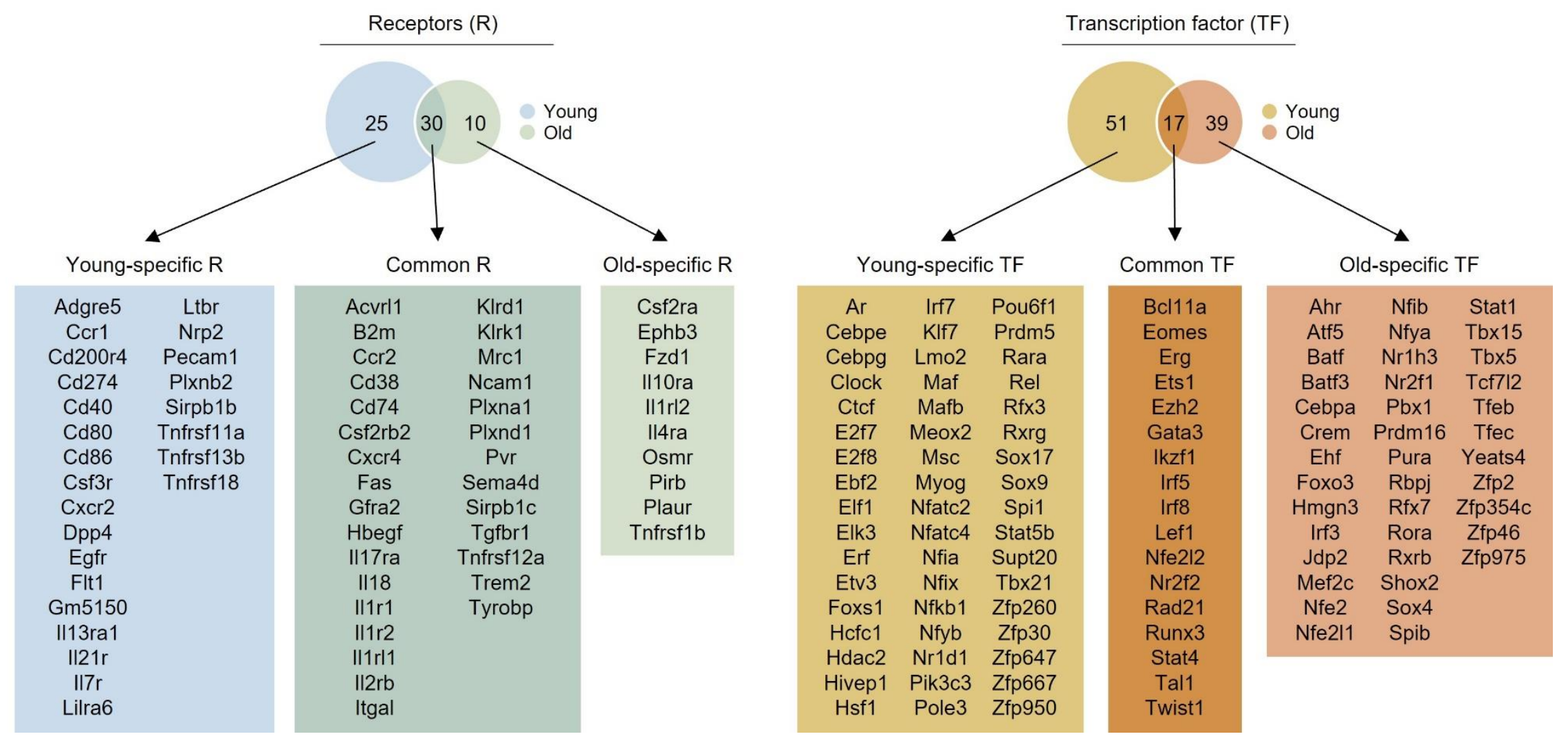

Extended data figure 11. A full list of receptors and transcription factors that are common or specific to young or aged animals using Domino. 


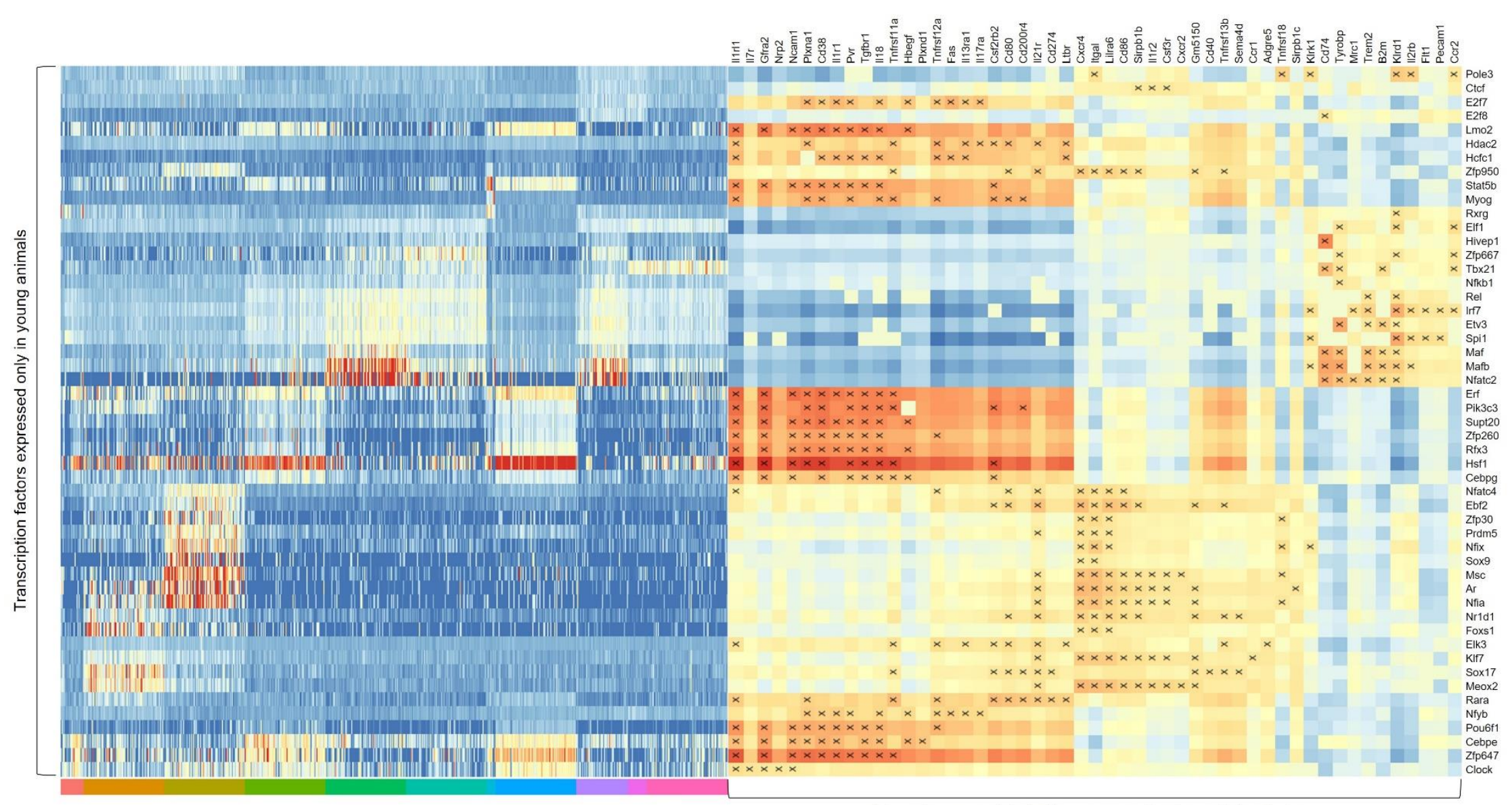

Receptors associated with corresponding transcription factors

Extended data figure 12. Heatmaps of activation score for young animal-specific transcription factors and their correlation with receptor expression. Transcription factors specific to young animals are labelled on the right, and the correlated receptors are labeled on top right. 


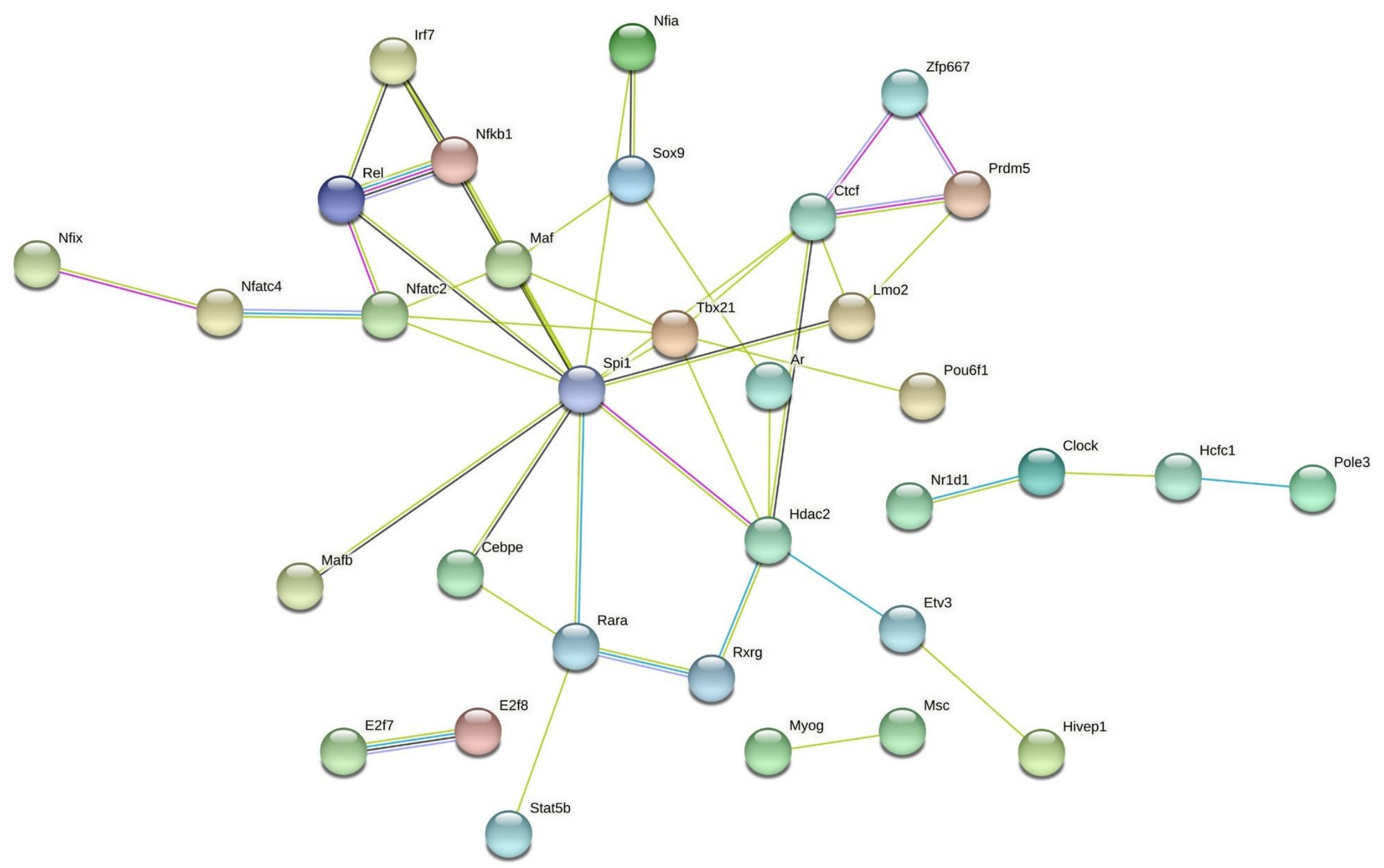

Extended data figure 13. Protein-protein interaction network (force-directed graph layout) for the transcription factors specific to young animals using STRING network. 

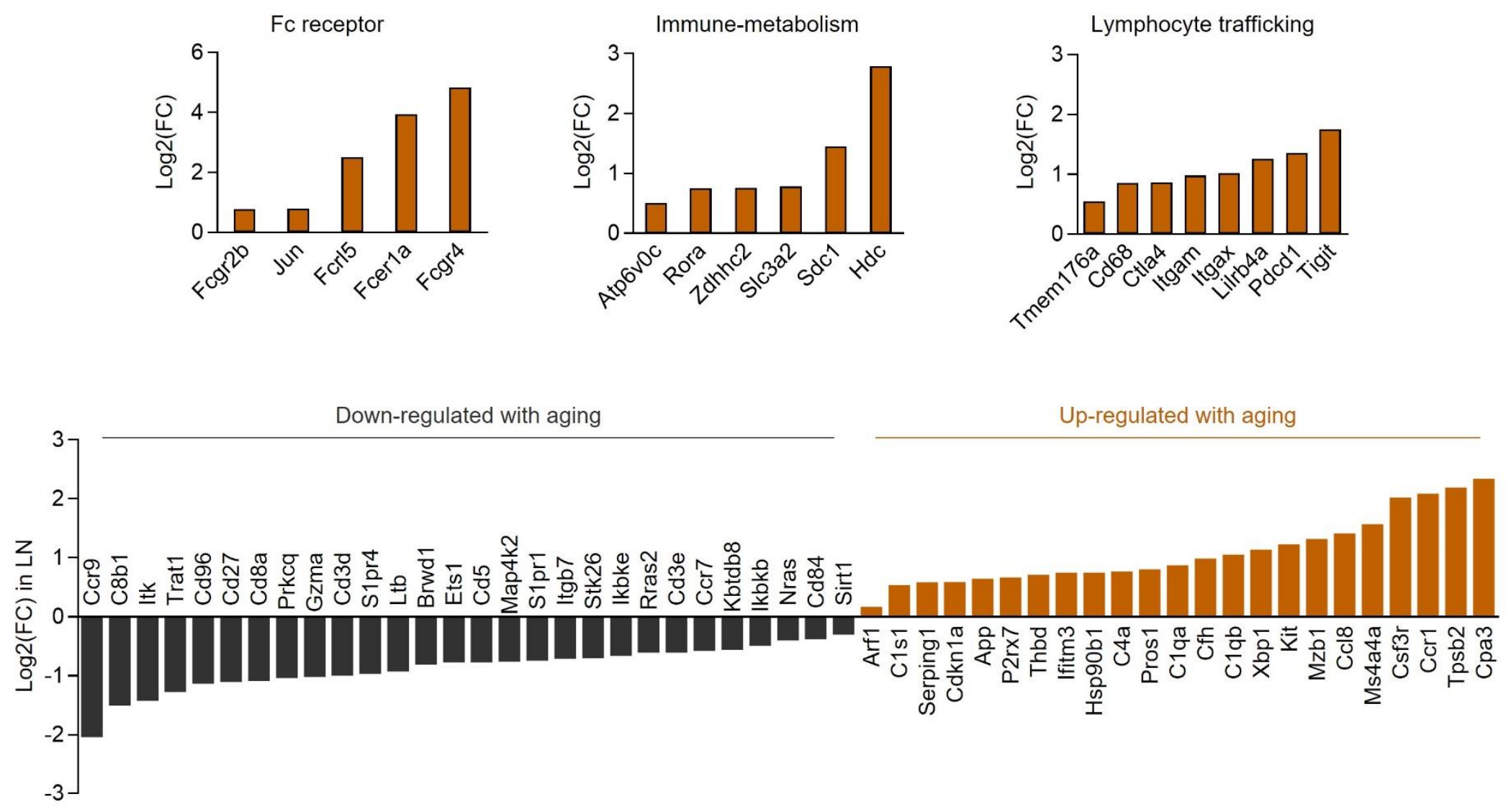

Extended data figure 14. A list of significantly up- or down-regulated genes $(p<0.05)$ in the aged lymph nodes compared to young lymph nodes from Nanostring analysis $(n=6)$. 

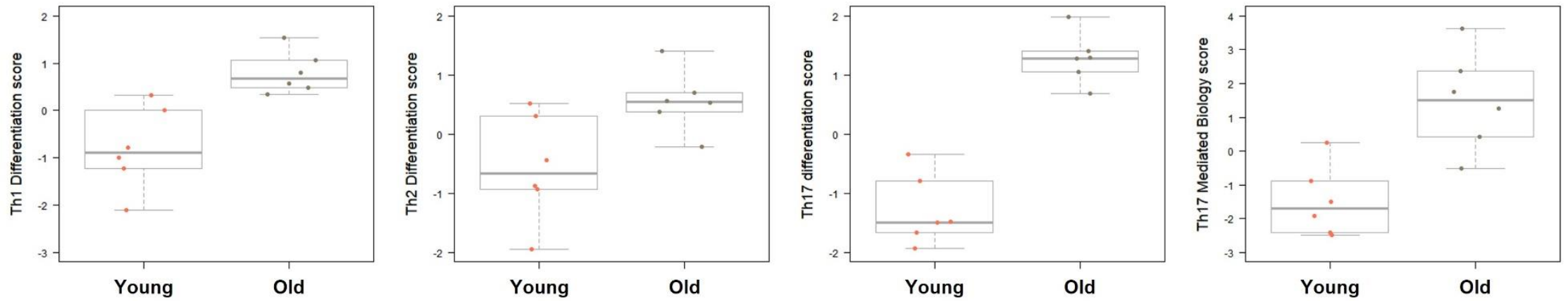

Extended data figure 15. Pathway scoring of the genes associated with Th1-, Th2-, Th17- differentiation or Th17-mediated biology from Nanostring analysis $(n=6)$. 
a.

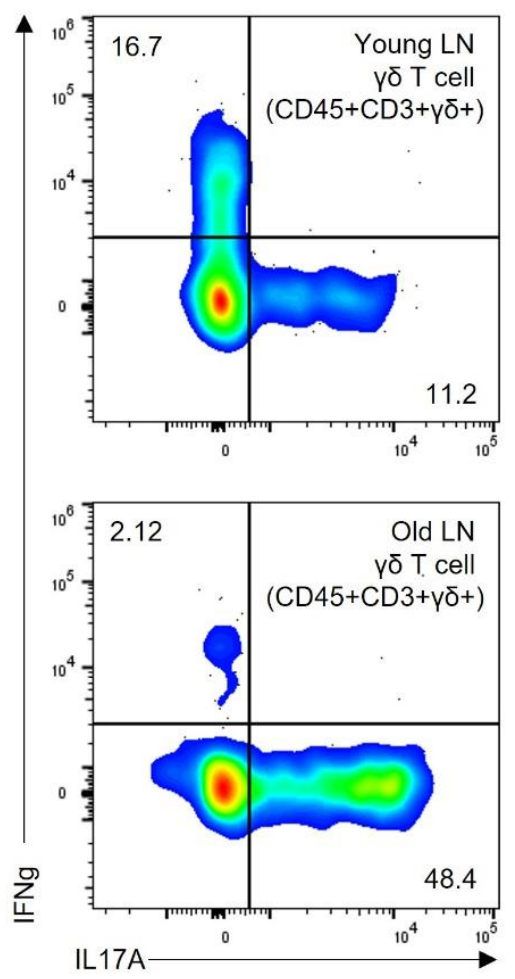

b.

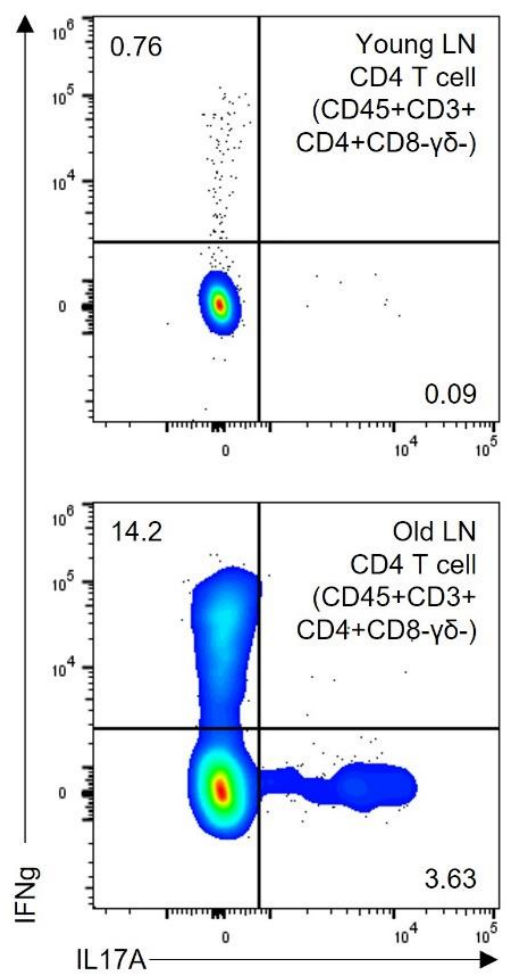

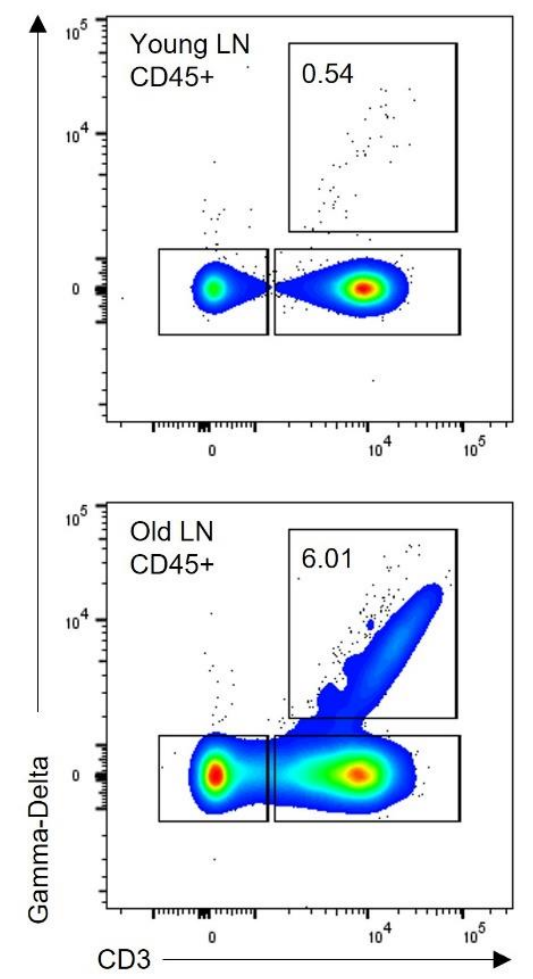

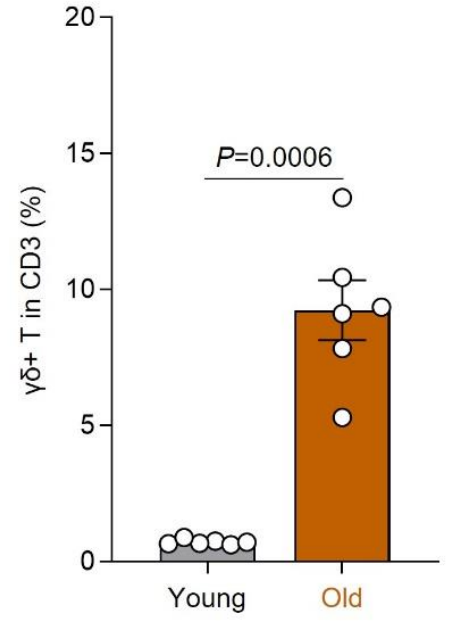

Extended data figure 16. Immune phenotype changes in the inguinal lymph node from young and aged animals without the treatments.

a, Representative images of flow cytometry data showing IFN $\gamma^{+}$or IL17A ${ }^{+} \gamma \delta$ or CD4 T cells in the lymph node. b, Representative images (left), and quantification of flow cytometry data showing $\gamma \delta \mathrm{T}$ cell population in $\mathrm{CD}^{+} \mathrm{T}$ cells in the lymph node ( $\mathrm{n}=6$ ). Unpaired two-tailed t-test (b). For all bar graphs, data are mean \pm s.e.m. 

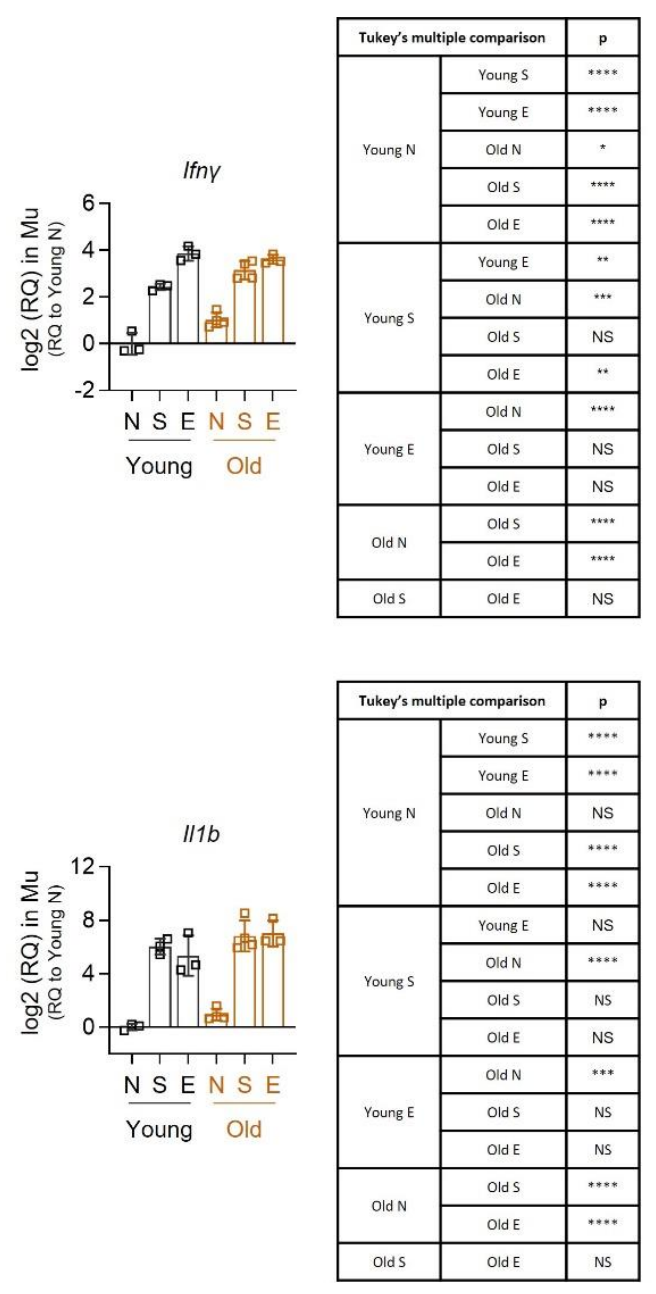
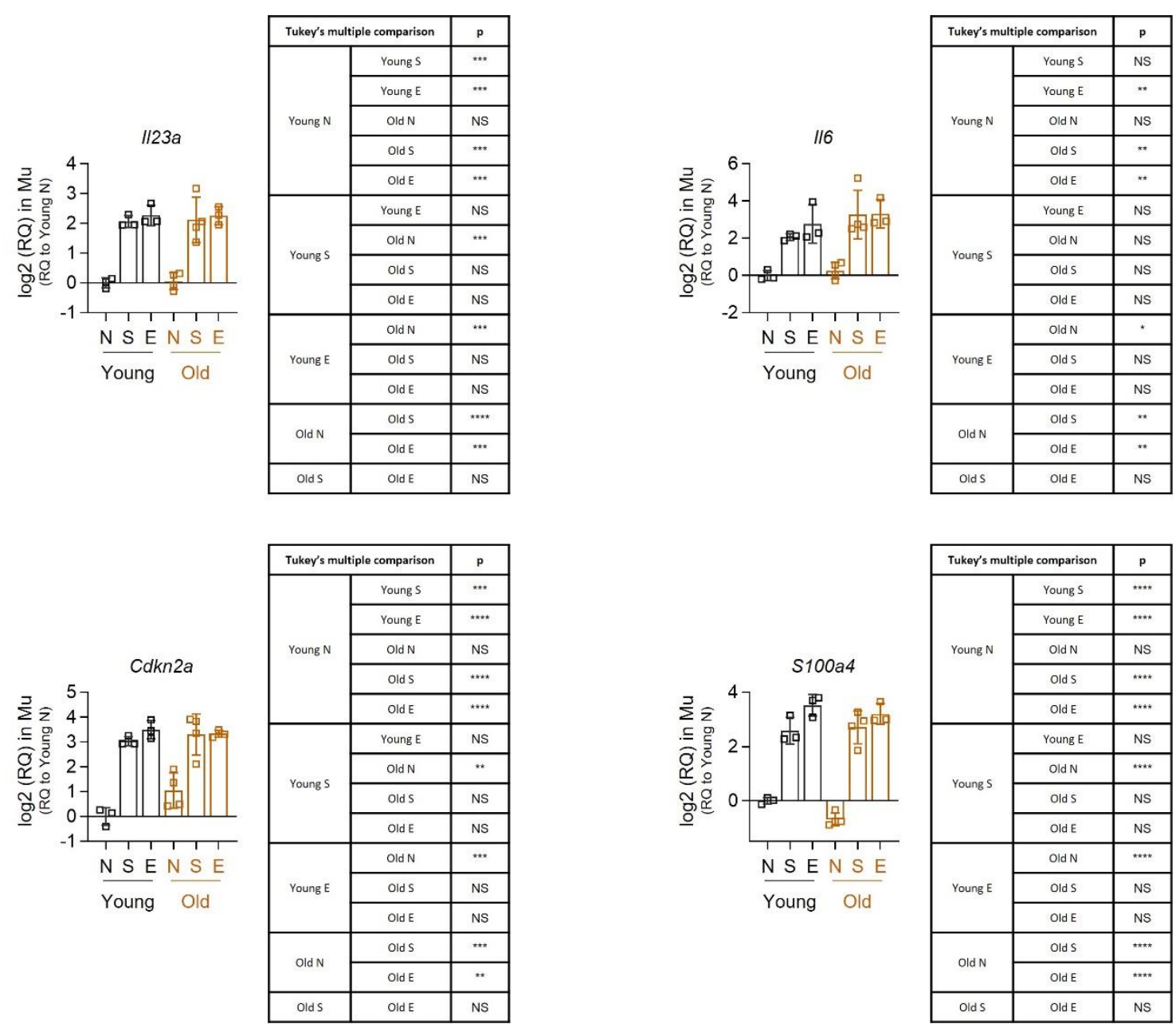

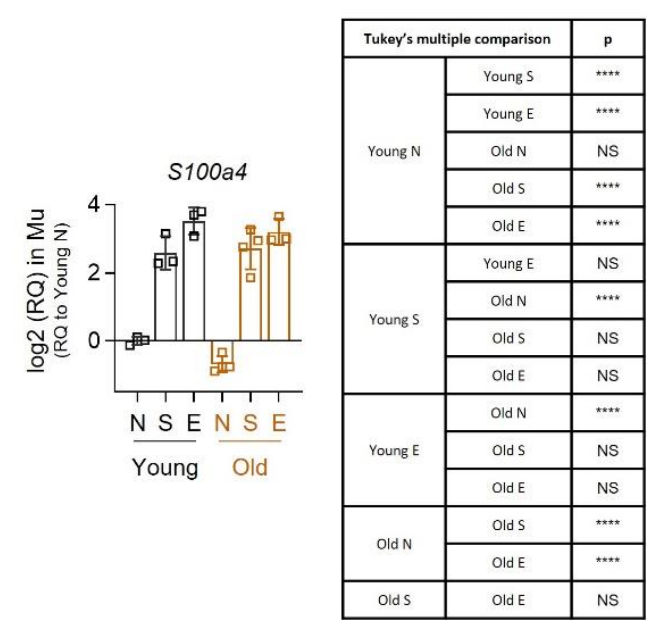

Experimental data figure 17. Quantification of inflammation- or senescence-associated genes in muscle 1 week after injury and treatment. Statistical analysis was performed using a two-way ANOVA with Tukey's multiple comparisons test $(\mathrm{n}=3-4)$. For all bar graphs, data are mean \pm s.d. 

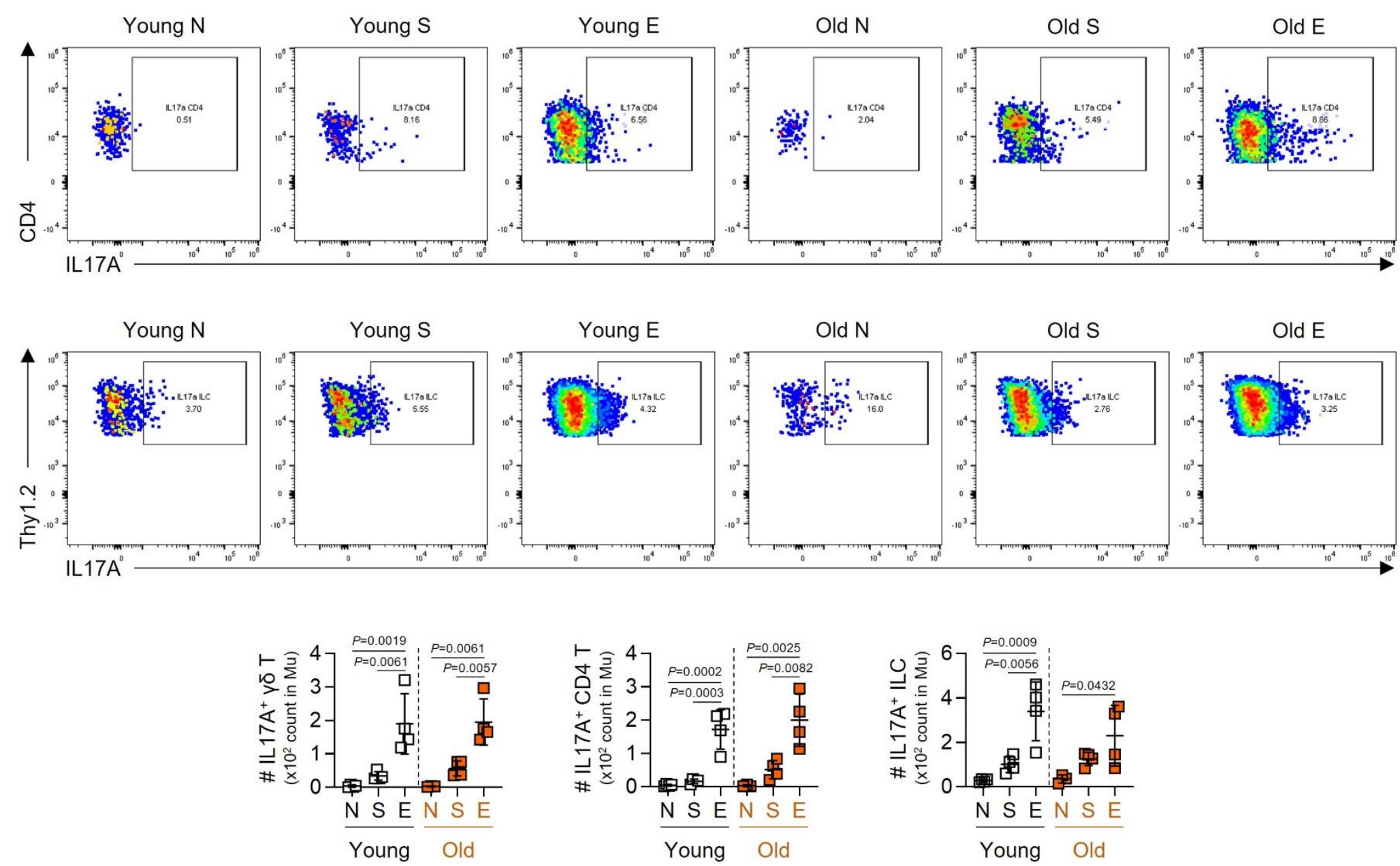

Extended data figure 18. Representative images of flow cytometry data showing IL17A ${ }^{+} \mathrm{CD} 4 \mathrm{~T}$ and innate lymphoid cells from young and aged animals (top) and the cell count quantification of IL17 producing cell subtypes in muscle 1 week after treatment (bottom). Statistical analysis was performed using a one-way ANOVA with Tukey's multiple comparisons test within the respective age groups $(\mathrm{n}=3-4)$. For all bar graphs, data are mean \pm s.d. 
Young No injury
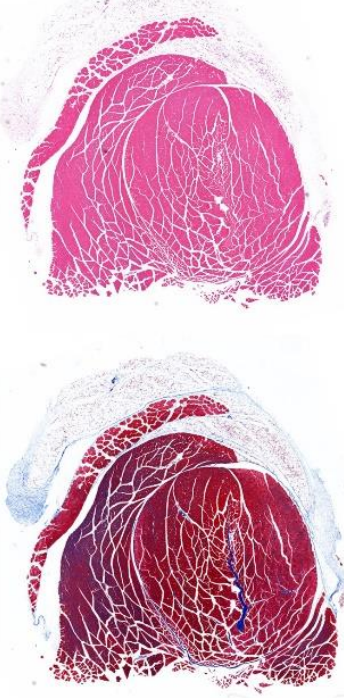

Young Saline 1wk
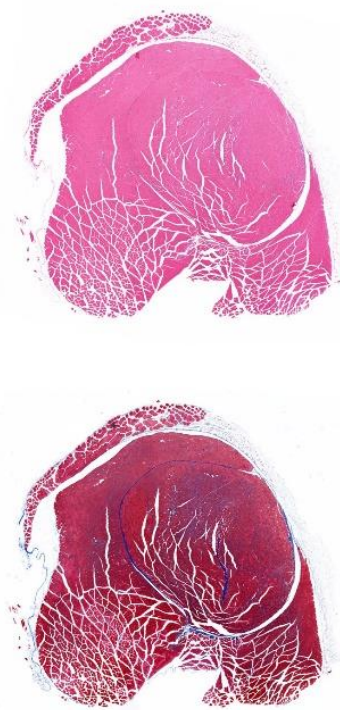

Young ECM 1wk
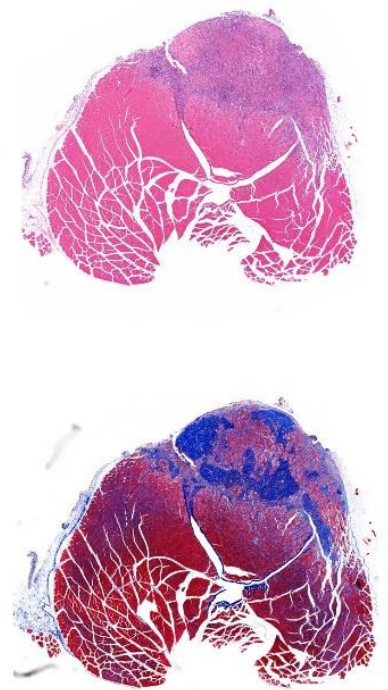

Old No injury

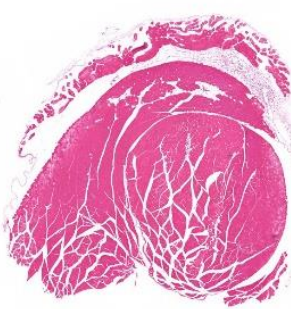

c.

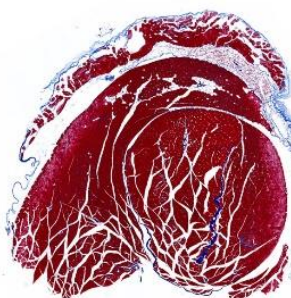

Old Saline 1 wk
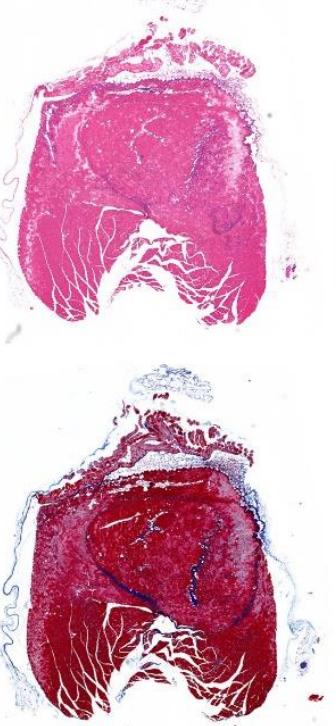

Old ECM 1wk
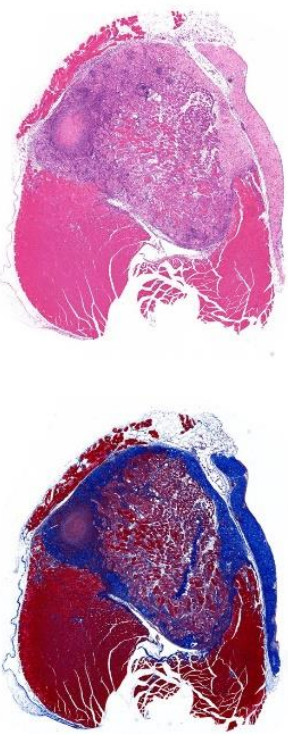

Extended data figure 19. Whole image of transverse section of the quadricep muscle 1 week after the treatments stained with H\&E (top) or Masson's Trichrome (bottom). 
a.

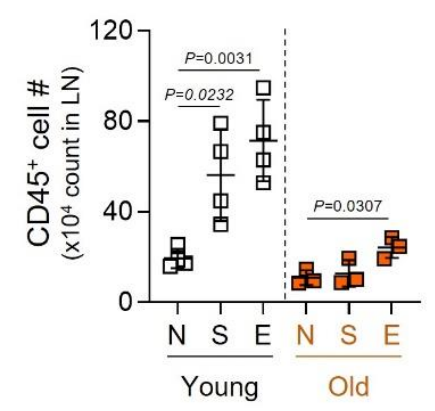

b.

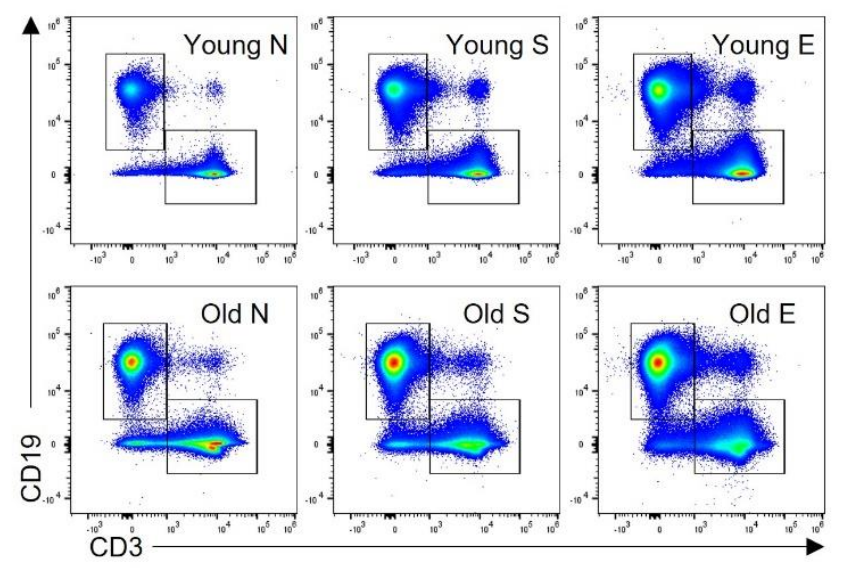

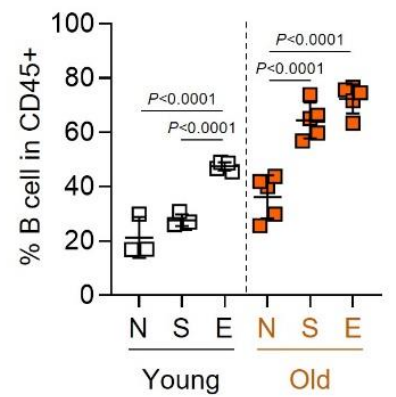

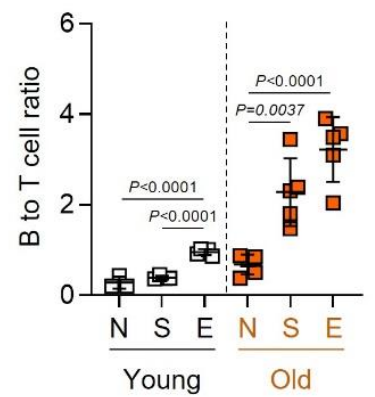

Extended data figure 20. Differential B cell response to ECM with aging. a, Cell count quantification of CD45 immune cells in the lymph node 1 week after injury and treatment. b, Representative images of flow cytometry data showing $\mathrm{CD}^{+} \mathrm{CD} 19^{-} \mathrm{T}$ cells or $\mathrm{CD} 3^{-}$ $\mathrm{CD} 19^{+} \mathrm{B}$ cells (left) and quantification of B cells and B/T cell ratio 1 week after the treatments (right). Statistical analysis was performed using a one-way ANOVA with Tukey's multiple comparisons test within the respective age groups (n=3-5). For all bar graphs, data are mean \pm s.d. 

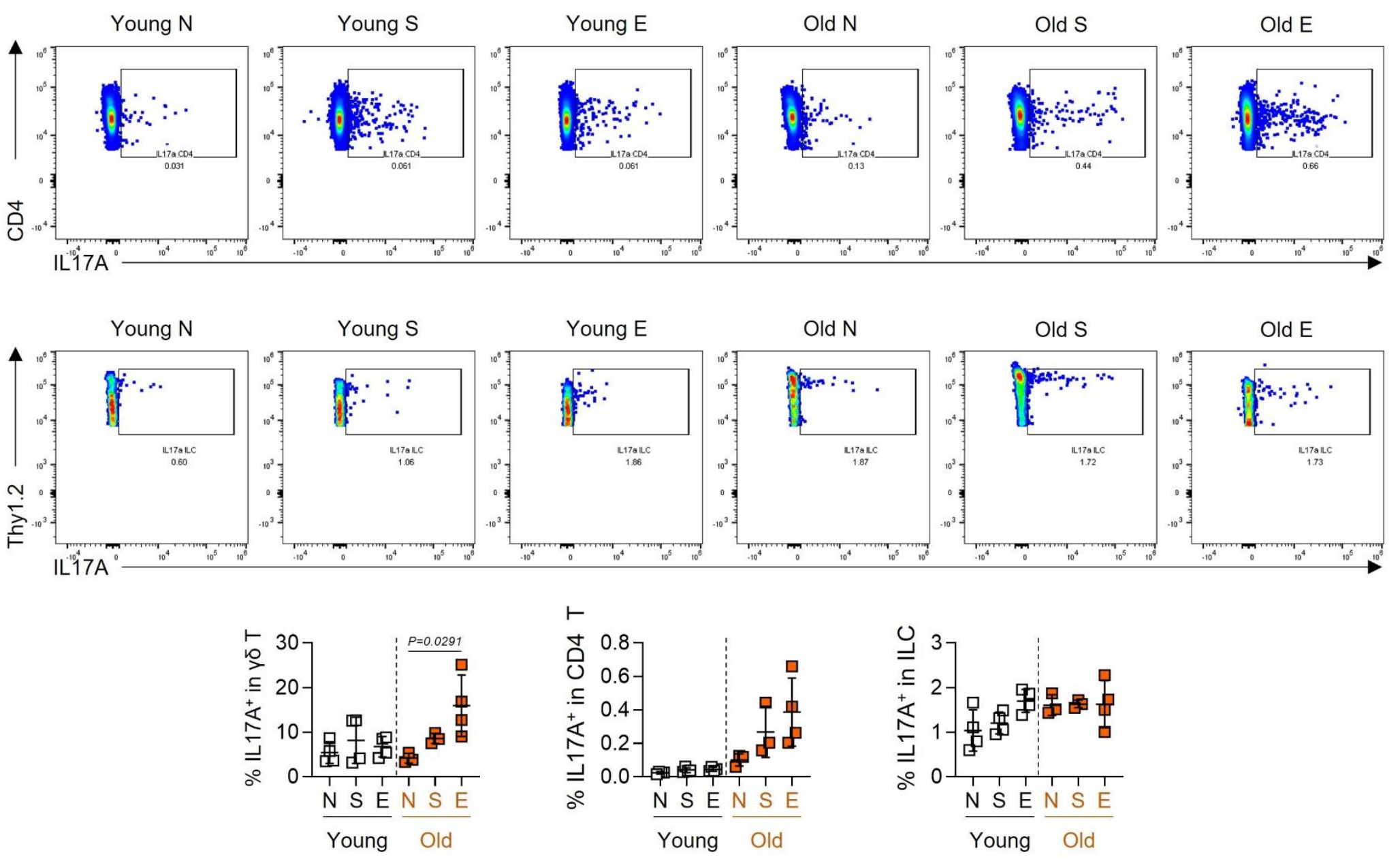

Extended data figure 21. Representative images of flowcytometry data showing IL17A ${ }^{+}$CD4 T or innate lymphoid cells between young and aged animals (top) and the cell percentage quantification of IL17 producing cell subtypes (bottom) in the lymph node 1 week after the treatments. Statistical analysis was performed using a one-way ANOVA with Tukey's multiple comparisons test within the respective age groups $(n=3-4)$. For all bar graphs, data are mean \pm s.d. 

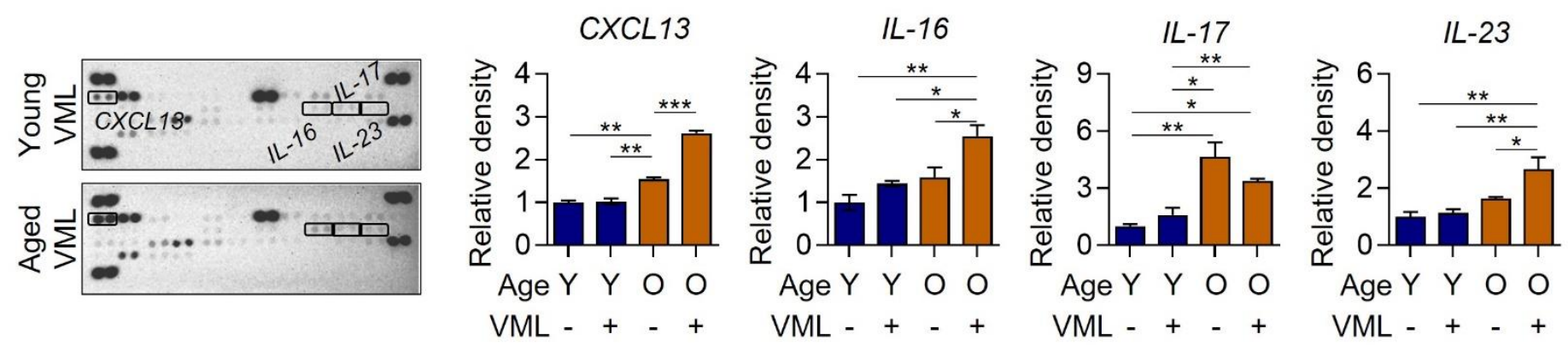

Extended data figure 22. Representative images (left) and quantitative analysis (right) of the protein array performed on blood serum from young and aged mice 1 week after injury. Protein molecules with significant differences in pixel densities between the groups are labeled and quantified using imageJ. Serum from 3 animals were pooled for analysis. Statistical analysis was performed using a Twoway ANOVA with Tukey's multiple comparisons test. ${ }^{*} p<0.05$, ** $p<0.01$, and $*^{* *} p<0.001$. For all bar graphs, data are mean \pm s.d. 
a.
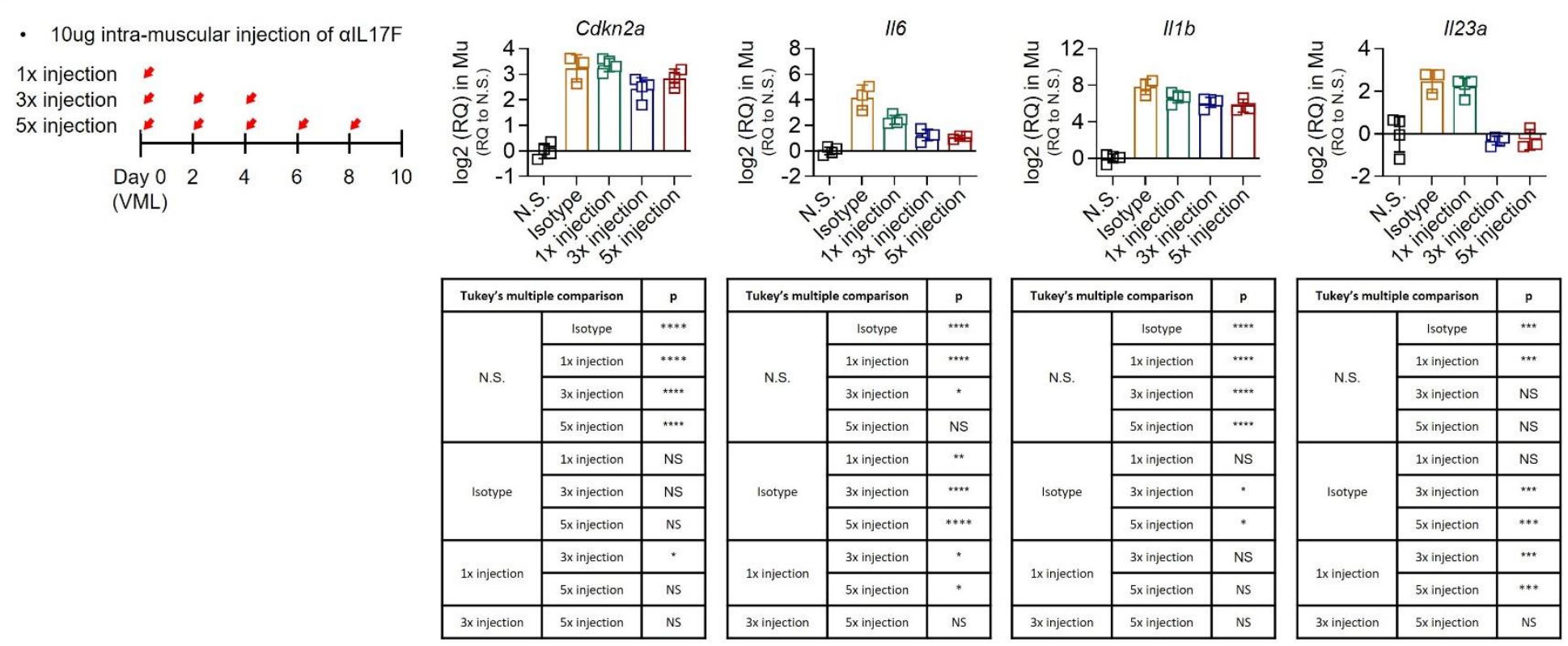

b.
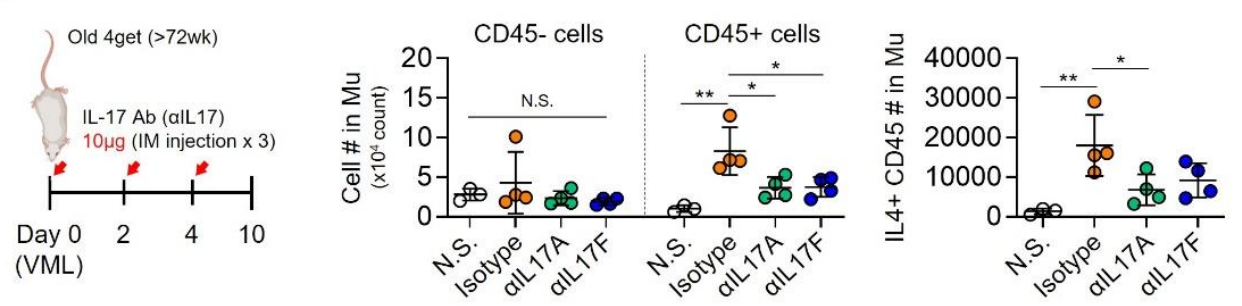

Extended data figure 23. Immune-modulatory effect of $\alpha \mathrm{IL} 17$ treatment. a, Schematic illustration of experimental design (left) and quantification of senescence- or inflammation-associated genes in muscle after various injection regime (right). b, Illustration of experimental design (left) and quantification of flow cytometry data showing the total number of $\mathrm{CD}^{-} 5^{-}$or $\mathrm{CD}^{4} 5^{+}$live cells or IL4 ${ }^{+} \mathrm{CD} 45^{+}$immune cells in muscle 10 days after VML injury (right). Statistical analysis was performed using a one-way ANOVA with Tukey's multiple comparisons test $(\mathrm{n}=3-4) .{ }^{*} p<0.05$, and $* * p<0.01$. For all bar graphs, data are mean \pm s.d. 

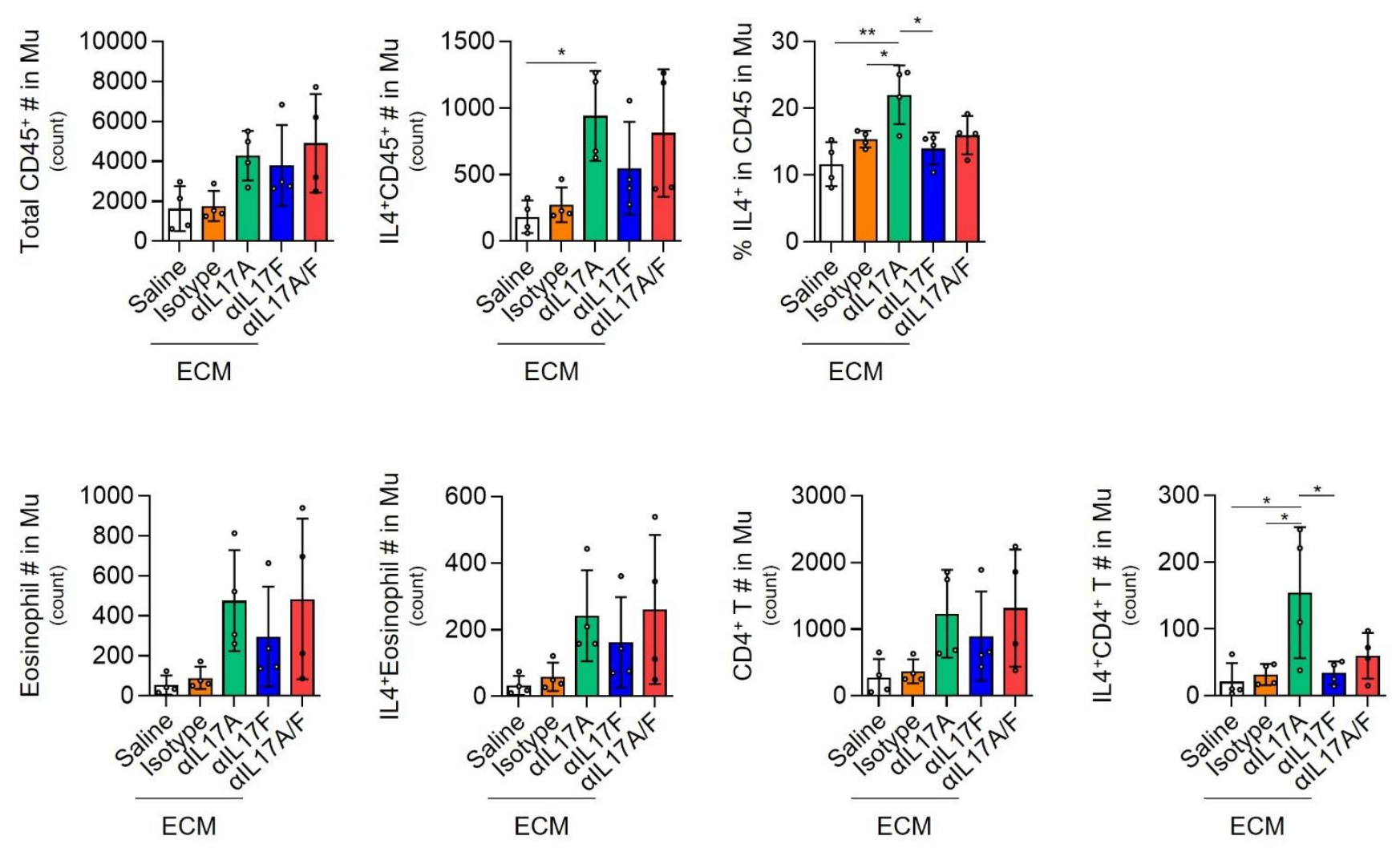

Extended data figure 24. Flow cytometry quantification of the indicated immune cell phenotypes in aged 4Get muscle 6 weeks after injury. Statistical analysis was performed using a one-way ANOVA with Tukey's multiple comparisons test $(\mathrm{n}=4) . * p<0.05$. For all bar graphs, data are mean \pm s.d. 

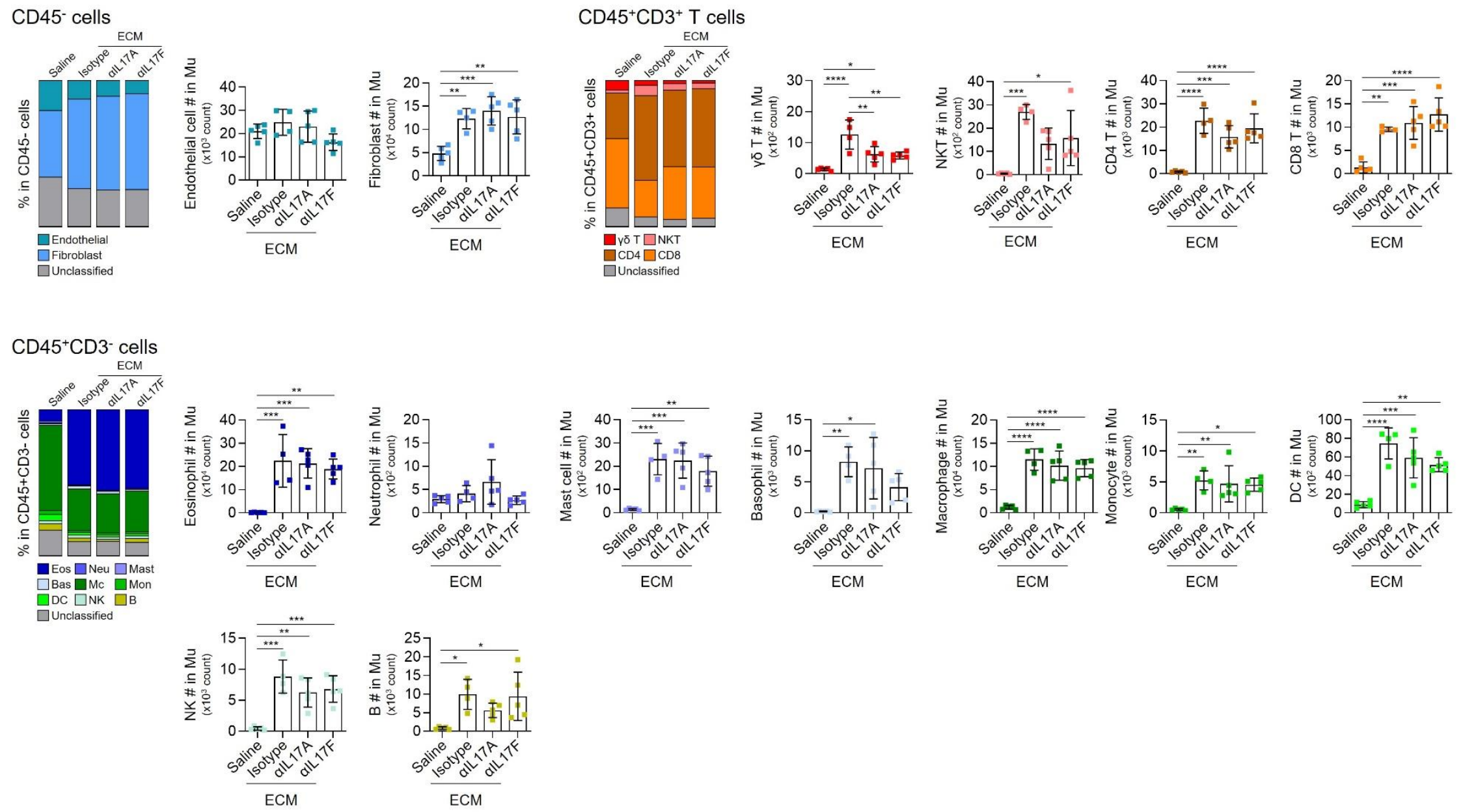

Extended data figure 25. Changes in immune and stromal cell phenotypes after $\alpha$ IL17 and ECM combination therapy in C57BL/6J aged animals. Tissues were analyzed 3 weeks after injury. Quantification of live cells in muscle showing the population of indicated cell phenotypes. Colored bar charts represent average cell count value of $n=4-5$. Statistical analysis was performed using a one-way ANOVA with Tukey's multiple comparisons test. $* p<0.05,{ }^{*} p<<0.01, * * * p<0.001$, and $* * * * p<0.0001$. For all bar graphs, data are mean \pm s.d. 

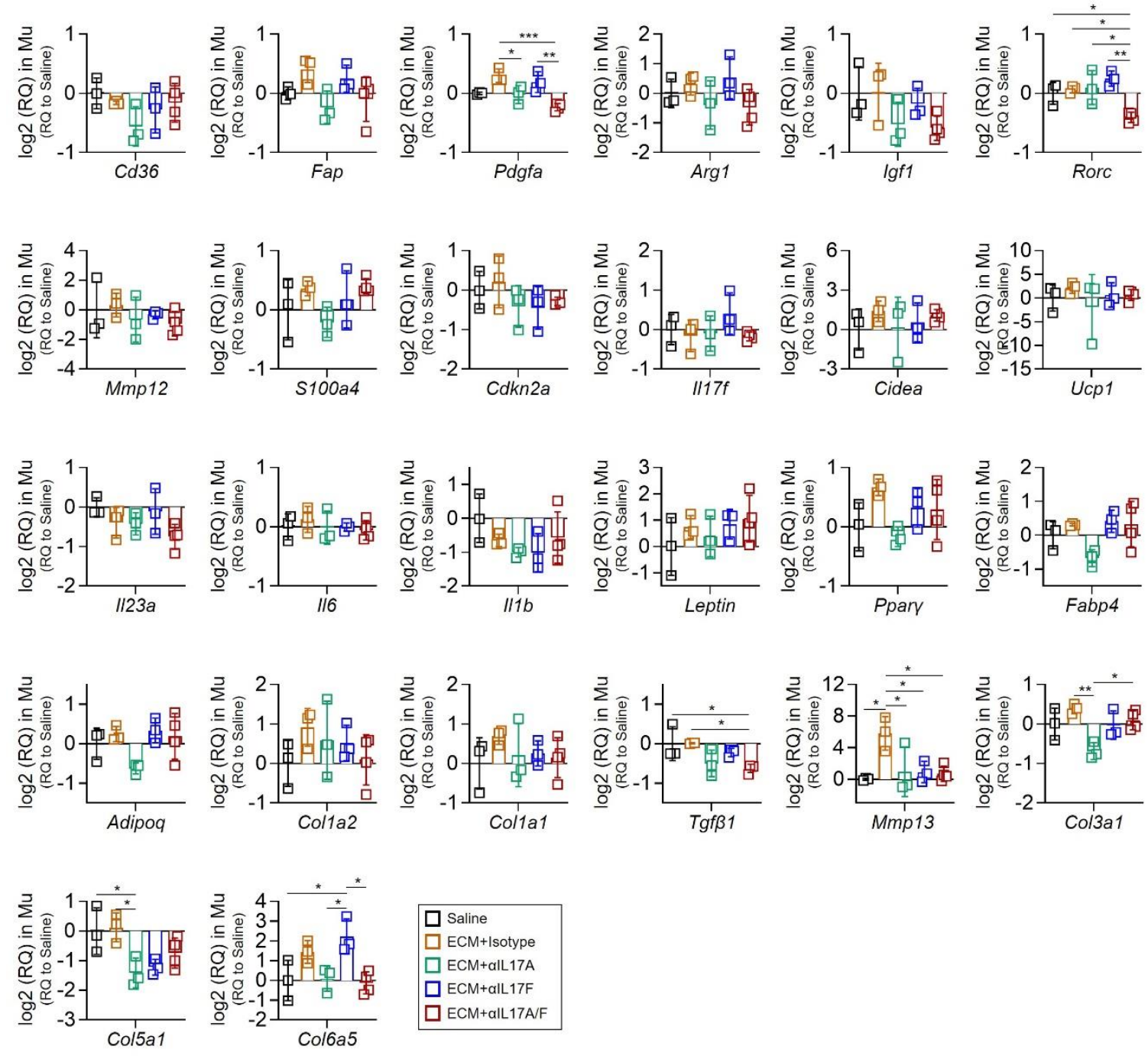

Extended data figure 26. Quantification of inflammation-, senescence-, adipose- or fibrosis-associated genes in aged C57BL/6J muscle 6 weeks after surgery. Statistical analysis was performed using a one-way ANOVA with Tukey's multiple comparisons test $(n=3-4)$. $* p<0.05, * * p<0.01, * * * p<0.001$, and $* * * * p<0.0001$. For all bar graphs, data are mean \pm s.d. 

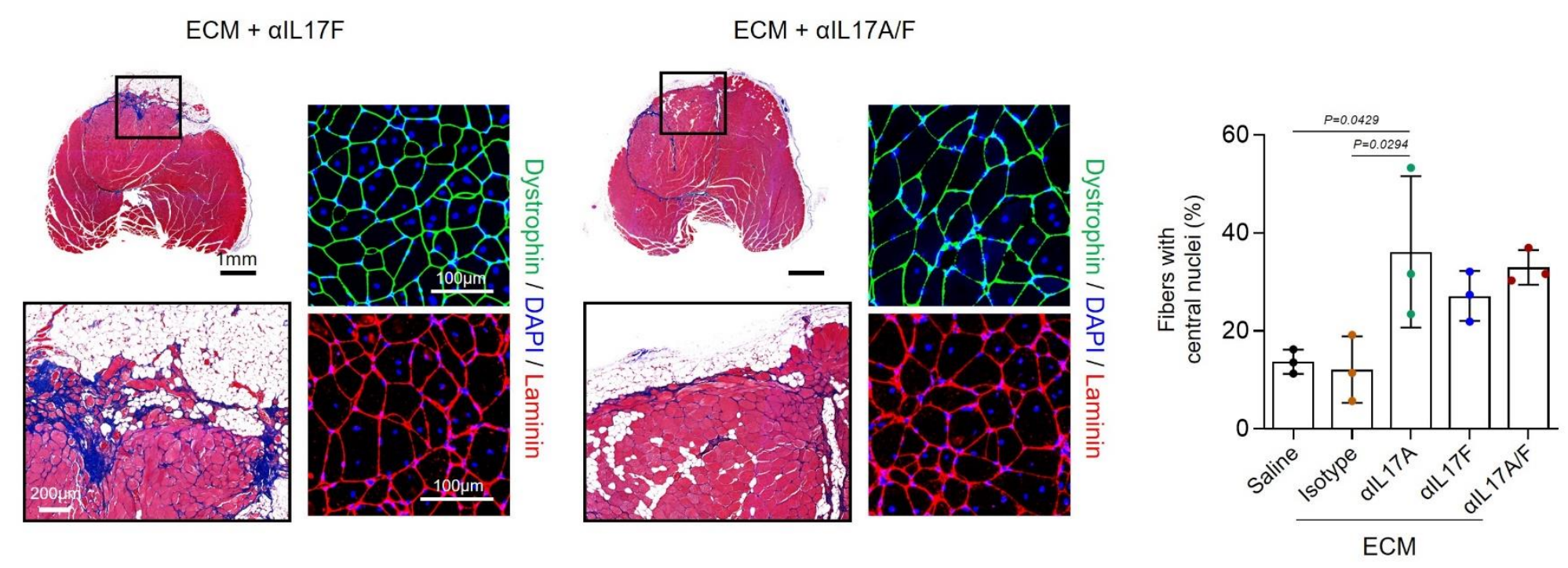

Extended data figure 27. Transverse section of the quadricep muscle 6 weeks after injury stained with Masson's Trichrome, dystrophin or laminin. Quantification of muscle fibers with central nuclei are shown. Statistical analysis was performed using a one-way ANOVA with Tukey's multiple comparisons test. For all bar graphs, data are mean \pm s.d. 


\begin{tabular}{|c|c|c|}
\hline \multicolumn{3}{|c|}{ Antibodies used for spectral flow cytometry } \\
\hline Fluorophores & Antigen & Clone \\
\hline BV421 & SiglecF & E502440 \\
\hline SuperBright436 & CD19 & 1D3 \\
\hline Pacific Blue & Ly6g & $1 \mathrm{~A} 8$ \\
\hline BV510 & CD31 & MEC13.3 \\
\hline BV570 & CD45 & $30-F 11$ \\
\hline BV605 & CD90.2 & $30-\mathrm{H} 12$ \\
\hline BV650 & Ly6c & HK1.4 \\
\hline BV711 & GD & GL3 \\
\hline BV750 & B220 & RA3-6B2 \\
\hline BV785 & $\mathrm{F} 4 / 80$ & BM8 \\
\hline BB515 & cKit & 2B8 \\
\hline SparkBlue550 & CD3 & $17 \mathrm{~A} 2$ \\
\hline PerCP & MHCII I-A/I-E & M5/114.15.2 \\
\hline BB700 & CD8 & $53-6.7$ \\
\hline PerCP-eFluor710 & CD29 & Hmb1-1 \\
\hline $\mathrm{PE}$ & CD115 & AFS98 \\
\hline PE-Dazzle594 & CD11c & N418 \\
\hline PE-Сy5.5 & CD68 & C68/684 \\
\hline PE-Cy7 & CD200R3 & BA13 \\
\hline AF647 & NK1.1 & PK136 \\
\hline AF700 & CD11b & $\mathrm{M} 1 / 70$ \\
\hline Zombie NIR & Viability & $\mathrm{N} / \mathrm{A}$ \\
\hline APC-Fire750 & CD36 & HM36 \\
\hline APC-Fire810 & CD4 & GK1.5 \\
\hline
\end{tabular}

\begin{tabular}{|c|c|c|}
\hline \multicolumn{3}{|c|}{ Antibodies used for $\mathbf{4 G e t}$ mice } \\
\hline Fluorophores & Antigen & Clone \\
\hline GFP & IL4 & N/A \\
\hline PerCP-Cy5.5 & CD3 & $17 \mathrm{~A} 2$ \\
\hline PE-Cy7 & $\mathrm{F} 4 / 80$ & BM8 \\
\hline PE594 & SiglecF & E502440 \\
\hline PE & CD8 & $53-6.7$ \\
\hline $\mathrm{APC}$ & CD4 & GK1.5 \\
\hline AF700 & CD11b & $\mathrm{M} 1 / 70$ \\
\hline BV605 & CD45 & $30-\mathrm{F} 11$ \\
\hline BV510 & Ly6c & HK1.4 \\
\hline Pacific Blue & Ly6g & $1 \mathrm{~A} 8$ \\
\hline eFluor780 & Viability & N/A \\
\hline \multicolumn{3}{|c|}{ Antibodies used for IL17A-GFP mice } \\
\hline Fluorophores & Antigen & Clone \\
\hline GFP & IL17A & $\mathrm{N} / \mathrm{A}$ \\
\hline PE-Cy7 & CD4 & GK1.5 \\
\hline PE594 & GD & GL3 \\
\hline PE & CD3 & $17 \mathrm{~A} 2$ \\
\hline BV605 & CD45 & $30-F 11$ \\
\hline Pacific Blue & CD90.2 & $30-\mathrm{H} 12$ \\
\hline eFluor780 & Viability & N/A \\
\hline
\end{tabular}

\begin{tabular}{|c|c|c|}
\hline \multicolumn{3}{|c|}{ Antibodies used for intracellular staining } \\
\hline Fluorophores & Antigen & Clone \\
\hline V500 & CD45 & $30-F 11$ \\
\hline BV711 & CD8 & $53-6.7$ \\
\hline AF488 & CD3 & 17 A2 \\
\hline PerCP-Cy5.5 & CD19 & 6D5 \\
\hline PE594 & GD & GL3 \\
\hline PE-Cy7 & CD4 & GK1.5 \\
\hline APC & IFNgamma & XMG1.2 \\
\hline AF700 & IL17A & TC11-18H10.1 \\
\hline eFluor780 & Viability & N/A \\
\hline
\end{tabular}

Extended data table 1. Antibody panel for the flow cytometry analysis. 